\title{
Shape-Programmed Folding of Stimuli-Responsive Polymer Bilayers
}

\author{
DISSERTATION \\ zur Erlangung des akademischen Grades \\ Doctor rerum naturalium \\ (Dr. rer. nat.) \\ vorgelegt \\ der Fakultät Mathematik und Naturwissenschaften \\ der Technischen Universität Dresden
}

von

Georgi Stoychev

geboren am 22.10.1985 in Veliko Turnovo

Eingereicht am

Die Dissertation wurde in der Zeit von 2009 bis

2013 im Leibniz Institut für Polymerforschung Dresden e.V. angefertigt. 


\section{Preface}

This work was carried out at the Leibniz-Institut für Polymerforschung Dresden e.V. in the years 2009-2013 under the supervision of Professor Manfred Stamm and Dr. Leonid Ionov. All experiments and analyses were carried out in the institute. The research was made possible by funding from the Leibniz-Institut für Polymerforschung Dresden e.V. as well as Deutsche Forschungsgemeinschaft DFG (Grant IO 68/1-1), which is gratefully acknowledged. 


\section{Outline}

The thesis is divided into four major parts. In the first chapter, a general introduction to the process of self-assembly and its importance in various emerging research fields is shortly outlined. The motivation for this work and its goals are also discussed.

The second chapter provides an extensive theoretical background on the self-assembly process and a comprehensive literature review on the most widely used folding and actuation systems. Actuation mechanisms, responsiveness to stimuli, fabrication methods as well as the applications of these systems are described, with a particular emphasis being put on swelling-based systems.

Detailed explanation of the experimental techniques and the analytical methods is given in the first part of Chapter 3. In the second part, the investigation results for each examined system and their discussion are represented. An overall of four major systems, bound to address specific problems, were examined. This work was the result of a continuous research, where the acquired results raised questions and led to new ideas. Therefore, for each system, a short motivation part is included, where the main questions and goals are stated. Technical details on polymer synthesis and sample preparation are then given and the results are represented and discussed. All the important observations and conclusions are then summarized.

Finally, the conclusions of this work and the possible further investigations as well as potential applications are presented in Chapter 4 . 


\section{Table of Contents}

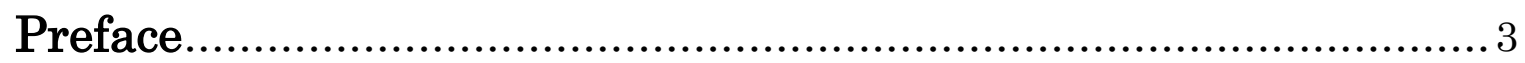

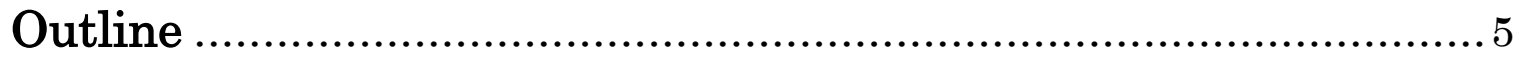

Table of Contents ........................................................................ 7

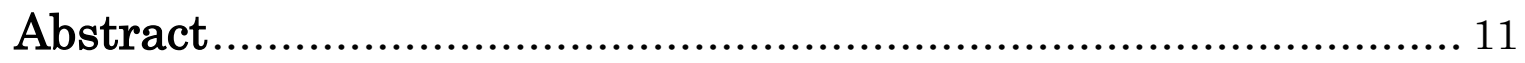

Motivation and Goals.......................................................... 15

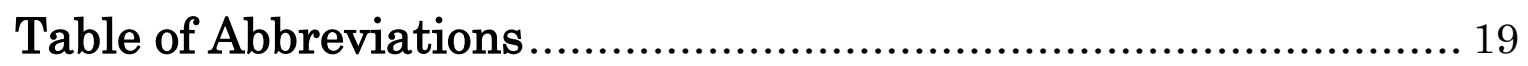

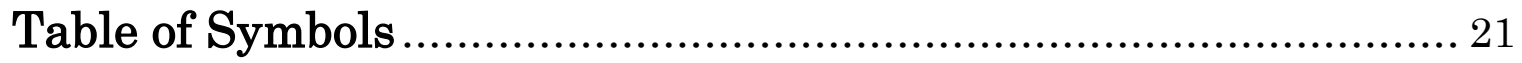

Theoretical Background ........................................................... 23

Wrinkling, Creasing and Folding - the Driving Principles of Self-

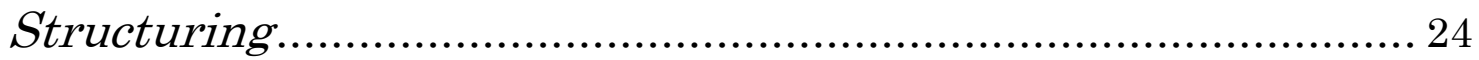

Fabrication of Wrinkled Surfaces............................................ 25

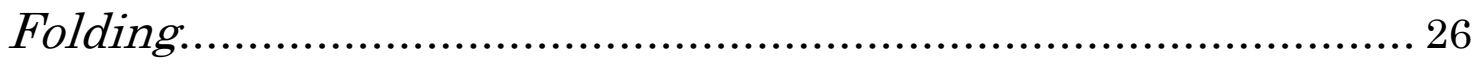

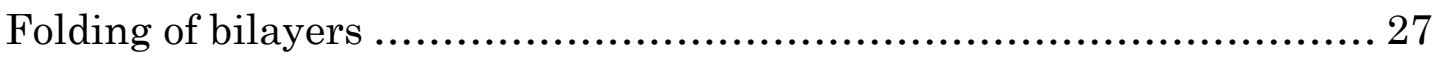

Actuation Mechanisms. Self-Folding, Actuating Devices and

Their Application. ................................................................... 30

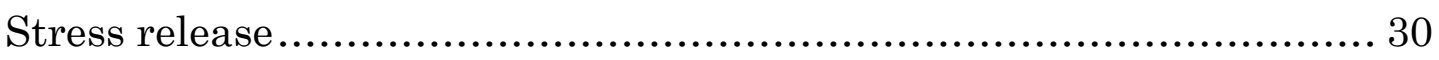

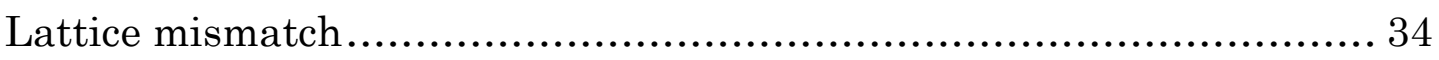

Surface tension as the driving force of folding ............................... 36

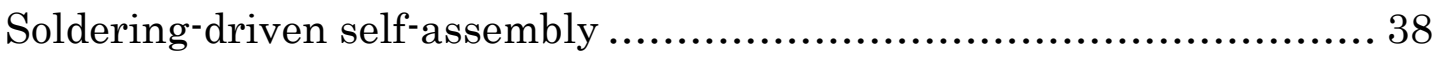

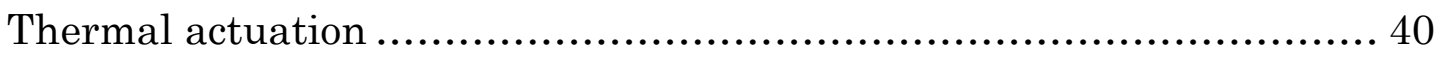

Shape Memory Polymers (SMPs) ….......................................... 42 
Polymers integrated with bio-actuators

$S_{\text {welling }}$.................................................................... 47

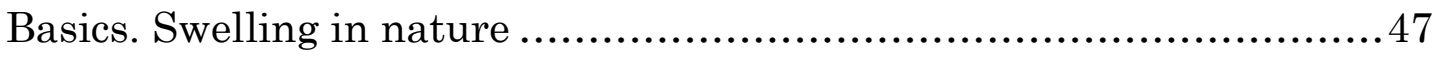

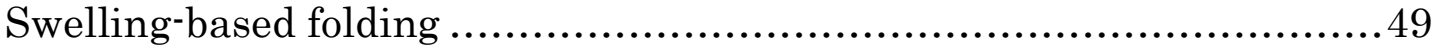

Stimuli-responsive self-folding films .................................. 49

Fabrication of Polymer Films.......................................... 53

Non-homogeneous swelling.............................................5 53

Homogeneous swelling ..................................................56

Applications ............................................................... 60

Complex Shapes .......................................................... 61

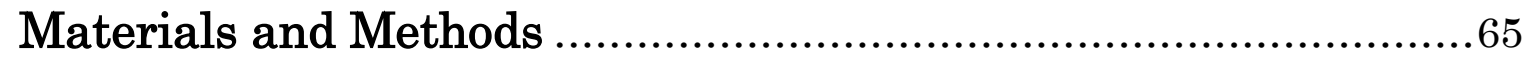

Materials................................................................... 65

Characterization Techniques.............................................. 71

Null-ellipsometry ...................................................... 71

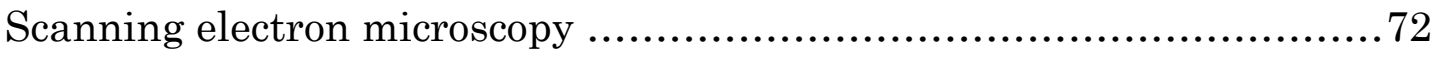

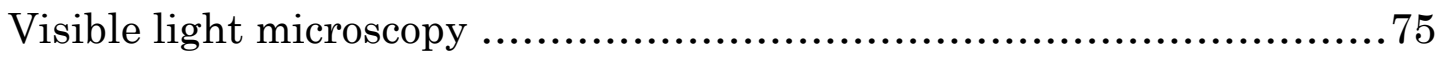

Results and Discussion .................................................. 79

Folding of Rectangular Bilayers ......................................... 79

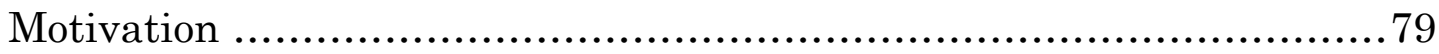

Experimental part..................................................... 80

Results and discussion ................................................... 81

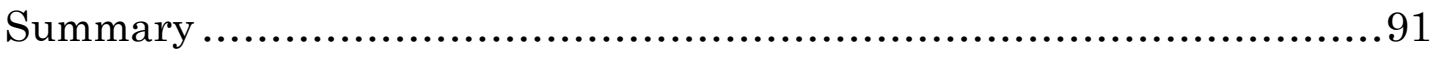

Folding of Complex Shapes. One-Step Folding........................92

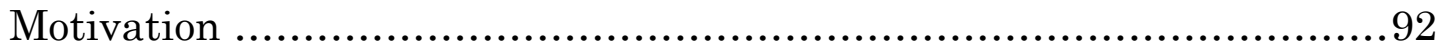

Experimental part....................................................... 93 


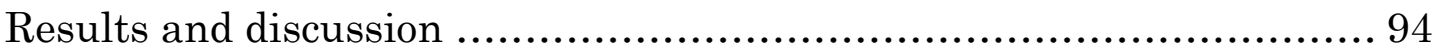

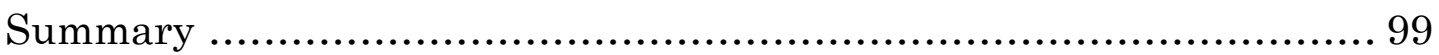

Folding of Complex Shapes. Multi-Step Folding...................... 100

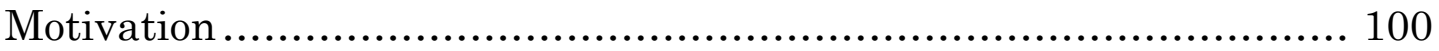

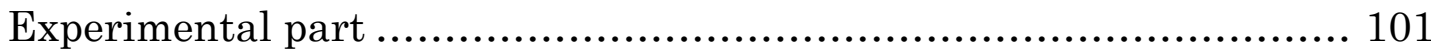

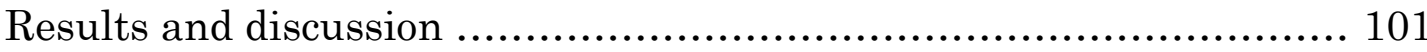

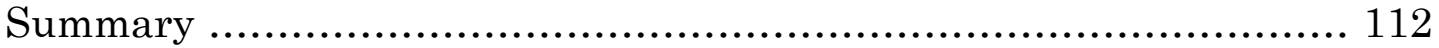

Multi-Step Folding of Porous Films ..................................... 113

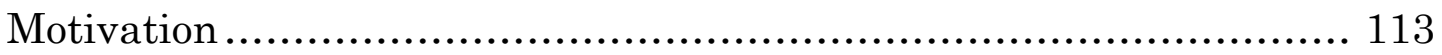

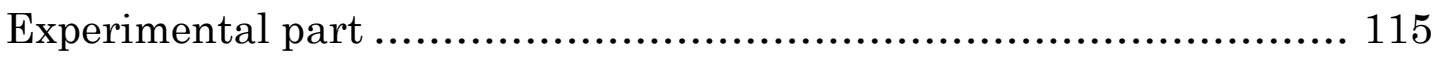

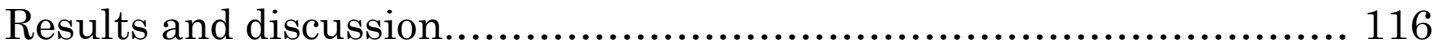

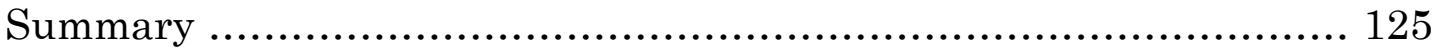

Summary and Conclusions ................................................ 127

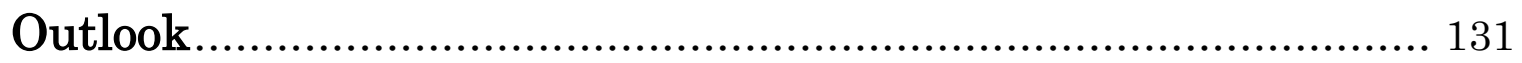

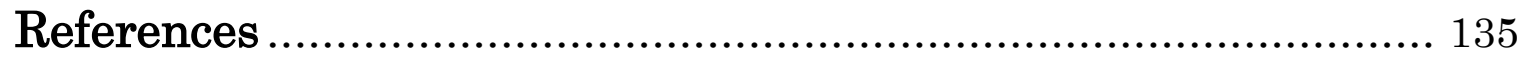

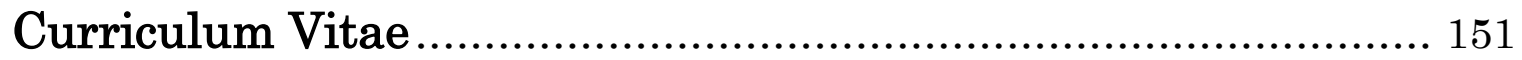

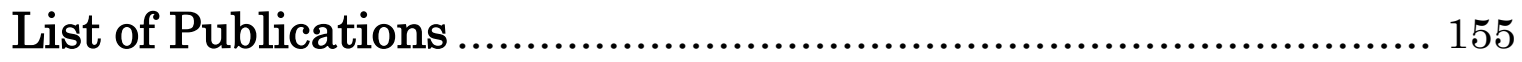

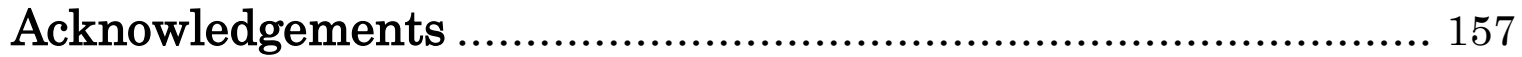




\section{Abstract}

$£$ Chapter

- Abstract

- Motivation and Goals

- Table of Abbreviations

- Table of Symbols
Self-folding polymer films are powerful prospective systems for the creation of various 3D constructs with promising applications in biotechnology, medicine, microfluidics, photovoltaics. Moreover, they were recently proposed as an alternative method for the design of three-dimensional constructs. On the other hand, due to the novelty of the approach, there is a noticeable lack in the amount of data on the behavior of such systems in the literature. This study is bound to fill the gaps and give a deeper insight into the understanding of how and why different types of folding occur.

In this study, four different types of folding of polymer bilayers are presented. Rectangles are one of the simplest geometrical forms and were therefore adopted as a convenient initial system for the investigation of the folding behavior of polymer bilayers. We 
chose PNIPAM for the active polymer, as it is a well-studied polymer with sharp Lower Critical Transition Temperature at around 33 C. For the passive layer, poly(methyl methacrylate) and poly(caprolactone) were chosen. The influence of different parameters of the system, such as polymer thickness and temperature was thoroughly investigated in order to be accounted for in later experiments. It was demonstrated that bilayers placed on a substrate start to roll from the corners due to quicker diffusion of water. Rolling from the long-side starts later but dominates at high aspect ratio. We showed that the main reasons causing a variety of rolling scenarios are (i) nonhomogenous swelling due to slow diffusion of water in hydrogels and (ii) adhesion of polymer to a substrate until a certain threshold. Moreover, nonhomogenous swelling determines folding in the first moments, while adhesion plays a decisive role at later stages of folding.

After having understood the abovementioned basics, we decided to explore how those applied to more complex shapes. For the purpose, four- and six-arm stars were chosen, the main idea behind this being the creation of self-folding polymer capsules capable of encapsulation of microparticles and cells. Adjusting the polymer thickness and thus the radius of folding allowed creating structures, capable of reversible self-folding and unfolding. The possibility to reversibly encapsulate and release objects in the micro-range was demonstrated on the example of yeast cells.

Noteworthy, the capsules were produced by means of the same process we used for the design of tubes - when compared to the folding of rectangles, it was the shape of the initial pattern and the folding radius that were changed; the mechanism was the same - simple one-step folding towards the center of the bilayer. Clearly the number of structures that can be generated by this method is fairly limited. The search for means to overcome this constraint led to the idea of hierarchical multi-step folding. Due to the edgeactivation of the bilayers, the observed deformed shapes differ from the classical ones obtained by homogeneous activation. It was found that films could demonstrate several kinds of actuation behavior such as wrinkling, bending and folding that result in a variety of shapes. It was demonstrated 
that one can introduce hinges into the folded structure by proper design of the bilayer's external shape through diffusion without having to use site selective deposition of active polymers. Experimental observations led us to derive four empirical rules:

1) "Bilayer polymer films placed on a substrate start to fold from their periphery and the number of formed wrinkles/tubes decreases until the angle between adjacent wrinkles/tubes approaches 130\%;

2) "After the wrinkles along the perimeter of the film form tubes, further folding proceeds along the lines connecting the vertexes of the folded film";

3) "The folding goes along the lines which are closer to the periphery of the films";

4) „Folding of the rays may result in blocking of the neighboring rays if the angle between the base of the folded ray and the shoulders of the neighboring rays is close to $180^{\circ}$ ".

These rules were then applied to direct the folding of edge-activated polymer bilayers through a concrete example - the design of a 3D pyramid.

One consequence of the second and third rules is that generally triangles are formed during the multi-step folding process. In order to create a cube, or any other 3D structure with non-triangular sides, an effective way to stop the folding along the lines, connecting neighboring vertexes had to be thought of. A possible solution would be the insertion of a rigid element inside the bilayer, perpendicular to the direction of folding. The solution of this problem was to design structures with pores. A pore would normally decrease the rigidity of a structure but in our case, a pore basically comprised an edge inside the structure and could thus form tubes which, as was already shown, exhibit much higher rigidity than a film. On the other hand, a pore, or many pores, would expose different parts of the active layer to the solvent and would strongly influence its swelling and, as a consequence, folding behavior. Hence, the influence of a pore on the swelling and the folding behavior of polymer bilayers had to be investigated. It was shown that pores of the right form and dimensions did indeed hinder the folding as intended. Instead, the 
polymer films took other ways to fold. As a result, despite the correctness of our reasoning, we failed to produce a cube by hierarchical folding of polymer bilayers. However, other sophisticated 3D objects were obtained, further increasing the arsenal of available structures, as well as giving an in-depth insight on the folding process.

This work provides the basic fundamentals of folding continuous as well as porous bilayers. Both theoretical considerations and experimental results have shown the possibility to create sophisticated shapes, suitable for various applications in medicine in biotechnology. This work can thus be considered a step towards the design of innovative materials. 


\section{Motivation and Goals}

The design of complex three-dimensional objects in the micro- and nanorange is a challenging and yet highly important task, because such structures have attractive potential uses in numerous areas, including microelectronics $1, \quad 2$, energy harvesting and storage photovoltaics ${ }^{3}$, microfluidics $^{4}$, design of biomaterials ${ }^{5-8}$, drug delivery ${ }^{9}$, 10, sensors. Conventional micromachining is difficult to downscale, is time-consuming and thus inappropriate for addressing the issue ${ }^{11}$.

There are two other general concepts for the creating of preprogrammed complex structures: self-assembly and self-folding ${ }^{12}$. In the self-assembly process many structural elements, or building blocks, are put together to form a larger, more sophisticated object (see Figure M1a). As one can imagine, the greater the number of building blocks, the more assembling possibilities exist. On one hand, many different structures can be designed by utilizing just few building blocks. On the other hand, by increasing the number of possible outcomes, the probability of obtaining exactly the required one decreases. In contrast, self-folding, the process of preprogrammed bending of a quasi-two-dimensional object into a three-dimensional one, offers a few, if not a single, final states but requires different pieces to be initially joined together (see Figure M1b). The reliability of the method has been used to produce numerous objects for cell-encapsulation ${ }^{13}$, drug-delivery ${ }^{10}$, microbots for use in medicine ${ }^{14,15}$, cell manipulation ${ }^{16}$.

Conventional self-assembly

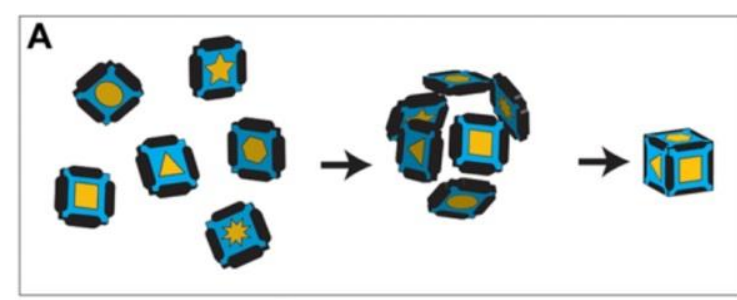

Self-folding

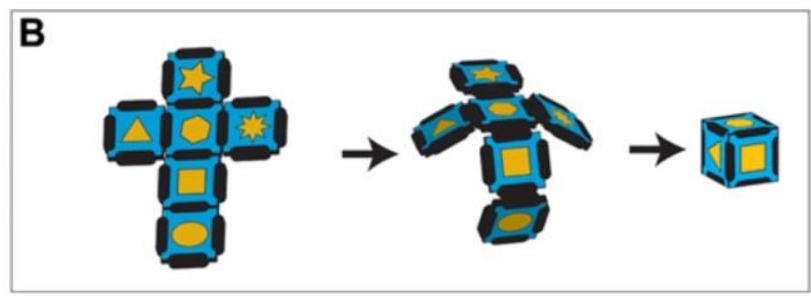

Figure M1. Two types of self-assembly ${ }^{17}$ 
A great number of different approaches to realize self-folding are described in the literature, including shape-memory materials ${ }^{18}$ that irreversibly change their conformation when exposed to external stimulus; multilayer films that bend upon heating due to release of the internal stress of one of the layers ${ }^{19}$; soldering of building blocks by surface tension ${ }^{2}, 7$; and localized volume change (shrinking) induced by heating ${ }^{20}$. Most of these methods employ inorganic materials, which, even though they provide mechanical robustness and stability, are not always biocompatible due to their toxicity or hardness and are therefore not suitable for biomedical applications. Moreover, many of these processes lead to structures capable of only one-time folding or assembling. Many fields, however, require microtools with the ability to reversibly change their shape in response to external stimulus. Polymers, on the other hand, seem to possess many appealing properties, such as lightness and softness combined with reasonable mechanical stability. Furthermore, polymers and especially co-polymers grant the possibility to easily functionalize and modify the material to increase its biocompatibility and to give it certain desired properties, such as stimuliresponsiveness. As a result, numerous polymers, which can respond to a certain stimulus, have been synthesized. Such stimuli include light ${ }^{21}$, temperature ${ }^{22,23}$, electric current ${ }^{24}$ and $\mathrm{pH}$. Recently, the use of temperatureresponsive, self-folding polymer bilayers was suggested as an alternative approach for the design of different 3D objects ${ }^{5}$. The driving force of the process is the swelling of one of the layers (called the active layer), while the second layer (also called the passive layer) swells to a much lesser degree or doesn't swell at all. As the two layers are conjugated, this inequality in swelling leads to bending of the polymer film.

A common feature of self-folding polymer structures is their inability to naturally form hinges and sharp edges - a homogeneous bilayer forms rounded bodies upon swelling. If hinges or edges are required, sophisticated, expensive and time-consuming preparation methods like multi-step photolithography have to be employed. 
In this work, two main goals were pursued. Firstly, to deepen the knowledge and understanding of the folding behavior of polymer bilayer films. Secondly, to explore the possibility of obtaining complex 3D constructs, based on self-folding polymer bilayers. Many works on self-folding systems report different and controversial bending scenarios. In this study we scrutinized the mechanism of folding of polymer bilayers in unprecedented depth. For this purpose, we started by considering simple geometrical forms, like squares and rectangles, and studying their swelling and folding nature. The influence of different parameters of the system, such as polymer thickness and temperature was thoroughly investigated in order to be accounted for in later experiments. Shape was then examined as a guiding factor for the manufacturing of sophisticated 3D assemblies. We also investigated the influence of holes of different size and shapes on the folding behavior of polymer bilayers, as it was expected that the introduction of holes in the polymer film could strongly influence the swelling scenario and should change the shape of the resulting structure. 


\section{Table of Abbreviations}

$\mathrm{AA}$

AIBN

BA

CSK

CTF

LCST

LT

MMA

MTFs

NIPAM

OPL

PBS

$\mathrm{PCl}$

PDMS

PEGDMA

PEGMA

PHEMA

PMAA

PMMA

PNIPAM

PTFE

PV

SEM

SMP

UV
Acrylic acid

N,N'-azobisisobutyronitrile

4-acryloyl benzophenone

Cytoskeleton

Cell traction force

Lower Critical Solution Temperature

Light-trapping

Methyl methacrylate

Muscular thin films

$\mathrm{N}$-isopropylacrylamide

Optical path length

Phosphate Buffer Saline

Poly(caprolactone)

Poly(dimethyl silocsane)

Polye(ethylene glycol) dimethacrylate

Poly(ethylenglycol methacrylate)

Poly(2-hydroxyethyl methacrylate)

Poly(methacrylic acid)

Poly(methacryl methacrylate)

Poly(N-isopropylacrylamide)

Poly(tetrafluorethylene), Teflon

Photovoltaic

Scanning Electron Microscope

Shape-memory polymer

Ultraviolet 


\section{Table of Symbols}

\begin{tabular}{|c|c|}
\hline$a_{L}$ & Lattice constant \\
\hline$a_{S}$ & Lattice constant of a substrate \\
\hline $\mathrm{C}$ & Circumference \\
\hline $\mathrm{d}$ & Resolution \\
\hline $\mathrm{D}$ & Diameter of folding \\
\hline $\mathrm{d}$ & Activation depth \\
\hline $\mathrm{E}$ & Elasticity modulus \\
\hline $\mathrm{F}$ & Elastic force \\
\hline $\mathrm{h}$ & Layer thickness \\
\hline $\mathrm{L}$ & Length \\
\hline M & Momentum \\
\hline NA & Numerical aperture \\
\hline $\mathrm{P}$ & Perimeter \\
\hline $\mathrm{r}$ & Radius of curvature (by folding) \\
\hline $\mathrm{T}_{\mathrm{g}}$ & Glass-transition temperature \\
\hline $\mathrm{W}$ & Width \\
\hline$\varepsilon$ & Film stress \\
\hline$\lambda$ & Wavelength \\
\hline
\end{tabular}




\section{Theoretical Background}

\section{Chapter}

\section{Theoretical}

\section{Background}

- Wrinkling, Creasing and Folding

- Fabrication of Wrinkled Surfaces

- Folding

- Actuation Mechanisms

- Swelling

- Fabrication of Polymer Films

- Applications

- Complex Shapes
The design of complex 3D architectures is a highly challenging task for the development of novel materials with improved optical properties, fabrication of tissue engineering scaffolds, as well as elements for micro- and nano-electronic devices. Nowadays, various methods for the construction of diverse threedimensional materials are available, which include twophoton photolithography, interference lithography, molding25. Recently, a novel and very appealing method for the fabrication of 3D microobjects was proposed (called microorigami ${ }^{25}, 26$ ), which is based on the programmed folding of two-dimensional structures into 3D ones. One of the advantages of this approach is the possibility of quick, reversible and reproducible fabrication of $3 \mathrm{D}$ hollow objects with controlled chemical properties and 
morphology of the both the outer and the inner side of the structure. The pioneering works in this field belong to Smella 27 and Jager 14, who worked with self-folding films and, ultimately, demonstrated the folding and unfolding of patterned gold films with polypyrole hinges in a response to an electric signal. The group of Oliver Schmidt expanded the approach and focused on the design of semiconductor and metal oxide self-rolled tubes, further applying them to transport cells ${ }^{28}$, the investigation of the behaviour of cells in confinement 29 , for nanooptics 30 and energy storage elements. Luchnikov et al designed self-rolling polymer tubes with patterned inner walls for cell encapsulation ${ }^{31}, 32$. Several approaches for the fabrication of metallic self-folding particles have been developed by Gracias et. al. ${ }^{33}$, who also demonstrated their applicability for the design of self-assembling microelectronic devices, controlled encapsulation of cells and drugs and as tissue engineering scaffolds1, 2, 8, 13. Moreover, metallic, self-folding thin films have also shown to be a promising material for optics ${ }^{34}$ and photovoltaic power applications ${ }^{3}$.

\section{Wrinkling, Creasing and Folding - the Driving Principles of Self- Structuring}

Self-structuring and self-organization are the driving principles of structure formation in nature. Therefore, they are of great interest due to their attractive implications in the design of novel intelligent materials ${ }^{35}$, 36. One example of self-assembling materials are thin polymer films with gradients of properties ${ }^{12,25}$.

Due to an inhomogeneous expansion/shrinking of different parts, such films are able to spontaneously form complex structures by wrinkling, creasing and folding. Thus, they mimic the mechanisms of fruit growth, plant movement and development of animal organs. Both creasing and wrinkling represent a bifurcation from a state of homogeneous deformation and typically occur when the substrate cannot be deformed (Figure T1a,b). 


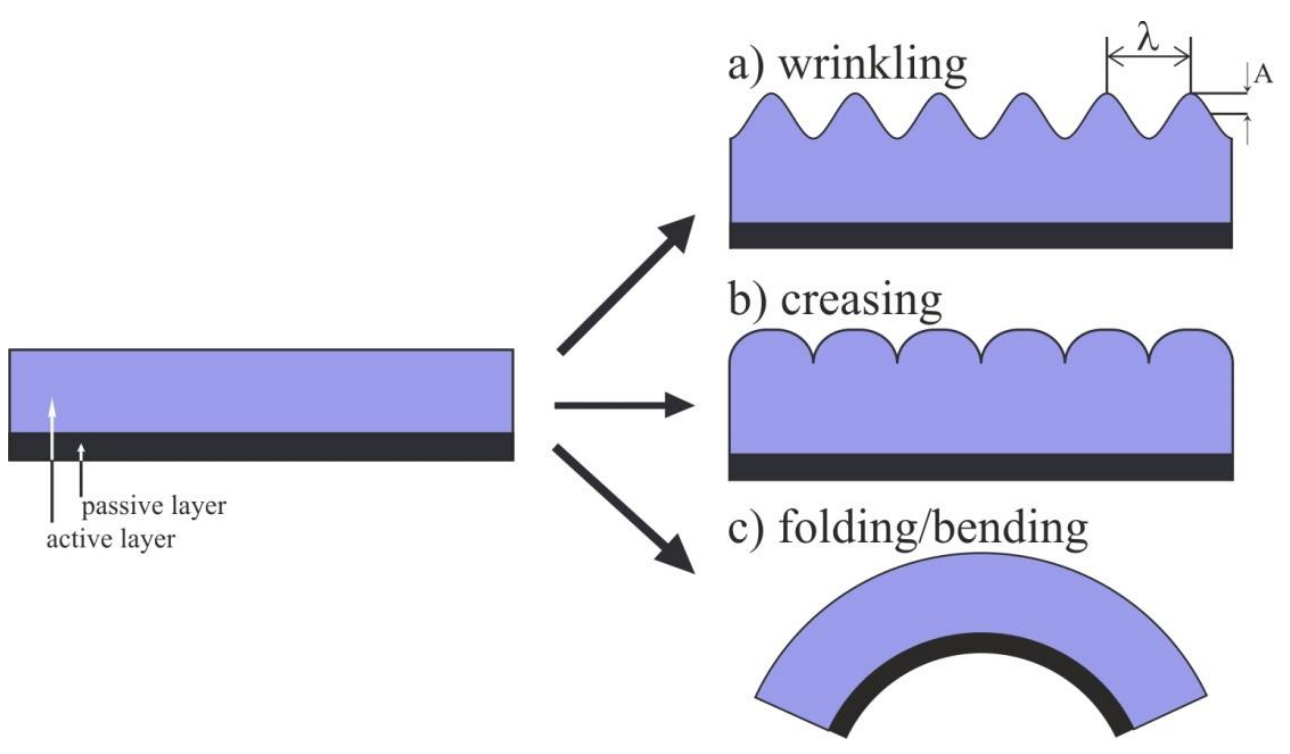

Figure T1. Different scenario of deformation of thin films: wrinkling, creasing and folding 12

Wrinkles, wherein the surface of the material undulates sinusoidally but remains locally smooth, involve a deviation from the homogeneous state by a field of strain that is infinitesimal in amplitude, but finite in space. Creases, in contrast, are localized folds that represent a deviation from the homogeneous state by a field of strain that is finite in amplitude, but infinitesimal in space. ${ }^{37}$ Folding or bending is, on the other hand, a macroscopic type of deformation that usually occurs when the passive layer is soft and deformable (Figure T1c). Utilization of these phenomena for the design of structured materials is highly attractive, as they allow a very simple fabrication of highly complex repetitive $2 \mathrm{D}$ and $3 \mathrm{D}$ patterns ${ }^{38-44}$.

\section{Fabrication of Wrinkled Surfaces}

Wrinkling and creasing may occur in two general cases of inhomogeneous films: the topmost layer either remains unchanged and the active layer shrinks ${ }^{45}, 46$, or the topmost layer swells to a greater extent than the bottom one 37, 47, 48 (Figure T2). The first scenario takes place when the film surface is photocrosslinked ${ }^{45,46}$ oxidized 49 , 50 or another substance is deposited on top of it, usually by means of vapour deposition ${ }^{51-53}$. The second case is observed, for example, during the swelling of a hydrogel with vertical 
gradient of crosslinking density and is associated with greater expansion of the top layer relative to the bottom layer 47,48 .
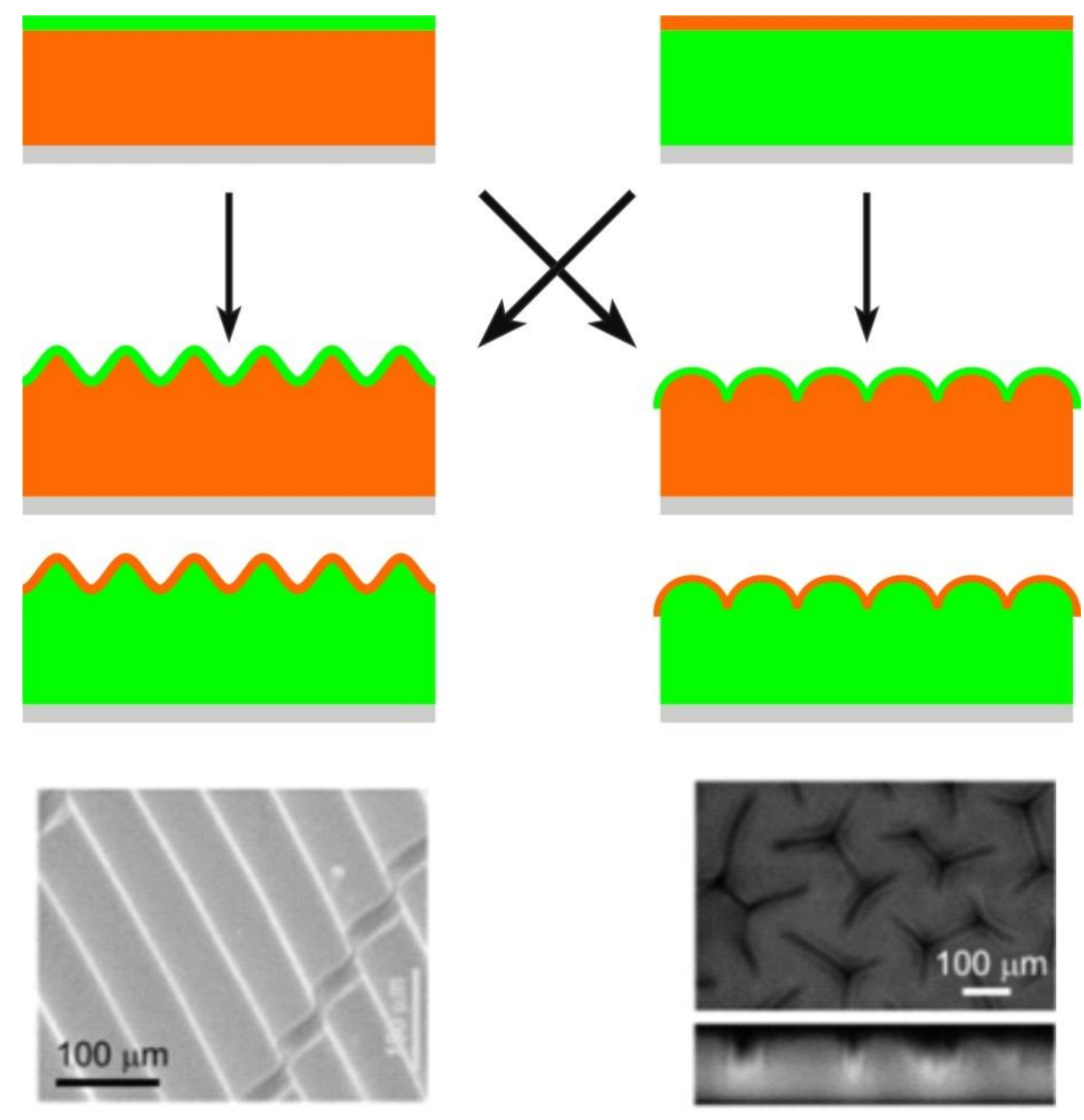

active layer

passive layer

Figure T 2. Two main approached for the generation of wrinkles and creases.

Wrinkling and creasing occur when the film is inhomogeneous and the topmost layer either remains unchanged and the active layer shrinks or the topmost layer swells stronger than the bottom one. Examples of wrinkles 54 and creases ${ }^{47}$

While the swelling mismatch is only one of the possible routes, another one would be the exploitation of the differences in thermal expansion of various materials ${ }^{55}$. Wrinkles can also be efficiently produced by a local deformation of free standing elastic membranes, which can be done using either a liquid drop or a needle ${ }^{56}$.

\section{Folding}

Folding, being of the same origin as wrinkling and creasing, represents an inhomogeneous change of volume. However, in contrast to wrinkling and 
creasing, which are commonly observed when the passive layer is rigid and inflexible, folding occurs when the passive layer is soft (Figure T 1,c). As a matter of fact, all these processes are interconnected, yielding a partly freehanging film (having one part fixed on a rigid substrate) to undergo transition from wrinkling to folding. For small strain gradients, the film wrinkles, while for sufficiently large gradients, a phase transition from wrinkling to bending occurs. ${ }^{57}$

Bending is an essential tool for the conversion of two-dimensional objects (films or layers) into 3D ones. Bending is typically observed as a result of either expansion or contraction of a material, caused by a change in the environmental conditions. However, a homogeneous expansion or contraction of an object in all directions preserves its shape and proportions. On the contrary, bending, as well as buckling and folding, is produced as a result of inhomogeneous expansion or shrinking, which occurs with different magnitudes in different directions. Similarly to buckling, which is a deformation of a film resulting in the appearance of regular wrinkles or irregular creases 42, 58-61, bending can be achieved by either applying field gradients to homogeneous materials or by applying non-gradient stimuli to inhomogeneous materials. An example of the first case is bending of a polyelectrolyte hydrogel in solution with a lateral gradient of $\mathrm{pH}$, which builds up during electrolysis ${ }^{4}$. Examples of the second group are bending of liquid crystalline films ${ }^{21}$, of hydrogels with lateral gradient in the monomer concentration ${ }^{62}$, or of cantilever sensors ${ }^{63}$.

\section{Folding of bilayers}

In 1925, Timoshenko ${ }^{64}$ considered the bending of metal bilayers which consist of two metals with different thermal expansion coefficients. At that point he assumed that the bilayer could bend in only one direction, which would result in a bilayer with a uniform curvature, thus

$$
\frac{1}{r}=\frac{6\left(\varepsilon_{2}-\varepsilon_{1}\right)(1+m)^{2}}{h\left(3(1+m)^{2}+(1+m n)\left(m^{2}+\frac{1}{m n}\right)\right)}
$$




$$
\begin{aligned}
& \frac{E_{1}}{E_{2}}=n \\
& \frac{h_{1}}{h_{2}}=m
\end{aligned}
$$

,where $E_{X}$ is the elasticity modulus of the layers, $\mathrm{h}_{\mathrm{x}}$ is the thickness of the layers, $h$ is the total thickness $\left(h=h_{1}+h_{2}\right), \varepsilon$ is the stress of the films, $\mathrm{r}$ is the radius of curvature. As equations 1-3 demonstrate, the radius of curvature is inversely proportional to the film stress. The radius of curvature first decreases and then increases with the increase of $m$. According to the equation, the radius of curvature is not very sensitive to the difference in stiffness between the two layers and is mainly controlled by the actuation strain and the layer thickness. The Timoshenko equation applies to a beam bending in only one direction and does not predict the direction of folding. Moreover, the Timoshenko equation is only applicable for elastic deformations.

More recent models have considered complex bending of a bilayer in two dimensions. Mansfield, for instance, found analytical solutions for large deflections of circular 65 and elliptical 66 plates having lenticular cross sections with a temperature gradient through the thickness. For small gradients, the plates formed spherical caps, curved equally in all directions. At a critical gradient, a configuration with greater curvature in one direction became more favorable. Because of the lens-shaped thickness profile, even though the elliptical plate had a major axis it showed no preferred direction for bending even for large deflections. Freund determined the strain at which the spherical cap, formed by circular bilayer of uniform thickness, becomes unstable using low order polynomial solutions and finite element simulations. 67

Later Smela et al. showed that short-side rolling was preferred in the case of free homogeneous actuation and that this preference increased with aspect ratio (the ratio of length to width of a rectangular pattern)68. Li et al. 69 and Schmidt ${ }^{70}$ experimentally demonstrated the opposite scenario, namely a preference for long-side rolling, in the case where bilayers are progressively 
etched from a substrate. They observed that, when the tube circumference was much larger than the width (incomplete rolling) or the aspect ratio of the rectangle was high (long stripes), rolling always occurred from the long side. When the tube circumference was much smaller than the width (tubes rolled several times) and the aspect ratio of the membrane pattern was not very high (in the case of a nearly square film), the rolling resulted in a mixed yield of long- and short-side rolling, as well as a "dead-locked turnover" shape. Short-side rolling occurred at small aspect ratios when the deformed circumference is close to the width of the film.

In inorganic self-rolling systems, the active component undergoes relatively small volume changes or actuation strains, which are nearly homogeneous over the whole sample. Hydrogels, however, demonstrate considerably different properties. Firstly, hydrogels are likely to undergo large volume changes (up to 10 times) upon swelling and contraction. Secondly, the swelling of a hydrogel is often kinetically limited: due to the slow diffusion of water through a hydrogel, the parts which are closer to the edges swell first, while the parts which are closer to the center of the film swell later.

Recently Hayward and Santangelo investigated the folding of patterned rectangular strips divided into one high- and one low-swelling region, which can be characterized as a thick but narrow bilayer 71 .

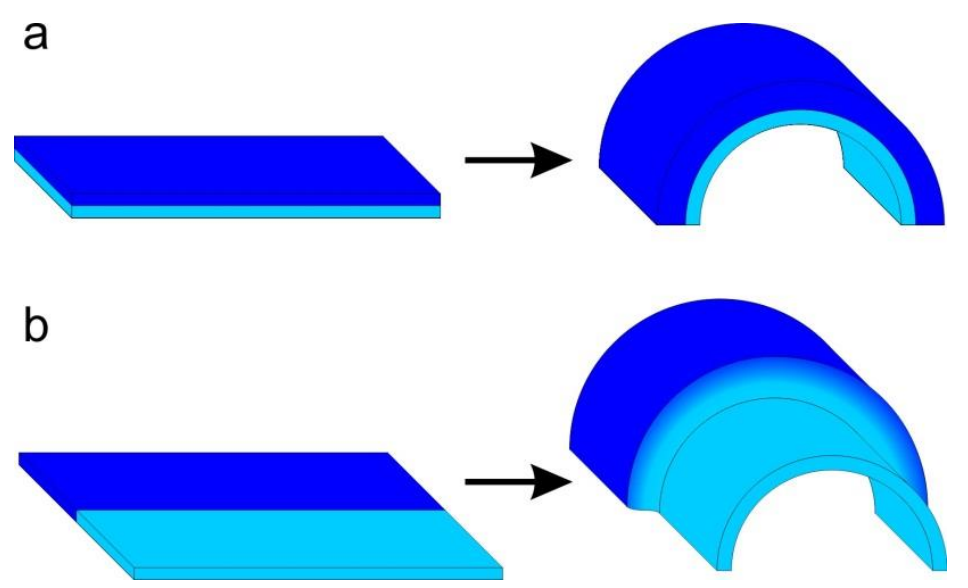

Figure T3. Bending of a "classical" bilayer (a) and a rectangular strip, divided into one high-and one low-swelling region (b). 
When swelled in an aqueous medium, it does not bend to the side of the low swelling component, as observed is the case of a "classical" bilayer structure (discussed by Timoshenko, see Figure T3a), but rolls into a threedimensional shape consisting of two nearly cylindrical regions connected by a transitional neck (Firgure 3b).

As a rule, the swelling of hydrogels is a relatively slow process, limited by the diffusion of water inside the polymer network, leading to folding/bending of bilayers that can occur in seconds or minutes. Recently, Bunning et al demonstrated the possibility of very fast deformation (ca 1/300 s) 72 on the example of liquid crystalline polymer films.

\section{Actuation Mechanisms. Self-Folding, Actuating Devices and Their Application}

\section{Stress release}

Residual stress is often found in thin polymer and metal blends. It is built up during the deposition process due to drying (polymers) or the formation of lattice mismatches (metals, inorganic films). Gracias et al. used mixed polymer-metal systems, where two thin metal films, namely copper and chromium, are thermally evaporated on the surface of a polymer layer. Here, folding of the structures is triggered by the release of the intrinsic stress that develops during the thermal deposition of the metals. The mismatch in the coefficient of thermal expansion of $\mathrm{Cu}, \mathrm{Cr}$ and the underlying polymer layer partly accounts for it, but it mostly results from the large intrinsic tension generated during the $\mathrm{Cr}$ film growth. At temperatures below its $\mathrm{T}_{\mathrm{g}}$, the polymer is hard enough to prevent the structure from folding. Heating it up leads to a decrease in its Young's modulus, thus enabling on-demand folding when heated. This process is schematically shown on Figure T4. Here, the internal hinges between the frames drive the folding process ${ }^{19}$. 
a)

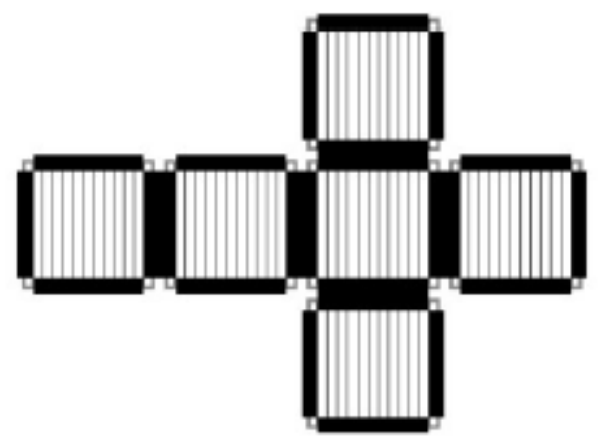

b)
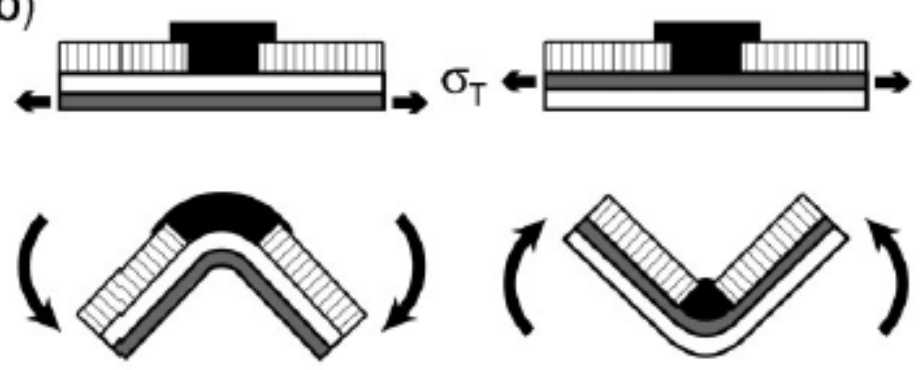

Legend

Chromium

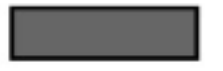

Photoresist

Copper

Nickel

Figure T4. Illustration of the structure and folding mechanism of stressrelease driven actuating devices; a) top view of a model structure; black lines denote polymer hinges; b) side view of part of the model structure; different order of metal deposition results in different folding direction ${ }^{19}$.

The $\mathrm{Cu}$ film provides stress mismatch with the $\mathrm{Cr}$ layer and enables the hinge to bend. Importantly, the position of the $\mathrm{Cr}$ layer determines the direction of folding. Placing it above the copper layer leads to upward folding, whereas depositing the $\mathrm{Cr}$ layer below results in bending in the opposite direction, i.e. the bilayer folds in the direction that reduces the lateral dimensions of the $\mathrm{Cr}$ layer (to relieve its residual tensile stress). The implementation of the process was shown on the examples of lowtemperature on-demand assembly of cubes, tetrahedrons and even more sophisticated polyhedra ${ }^{19}$.

This method was further expanded by introducing two, instead of only one, polymers to different joints of the abovementioned microstructures. For this purpose, six-arm grippers with four joints per ray were produced. One of 
the joints of each arm was covered with one type of a polymer, whereas all the others - with a different. As in the previous case, the polymer blend arrested the stress-release in the metal layer.

Here, both polymers were natural biopolymers and were therefore attacked and degraded by enzymes. First, an enzyme specific for one of the polymers was added to the media, which led to its softening and the stress release resulted in folding of the microgripper. Joints covered with the second polymer, however, were not influenced by the enzyme and retained their innate strain. Moreover, these joints were designed in a way that, if the polymer softens and stress is released, bending would occur in an opposite to the bending direction. If an enzyme that gauges the second polymer was then added, the microgrippers opened (see Figure T5) ${ }^{73}$.

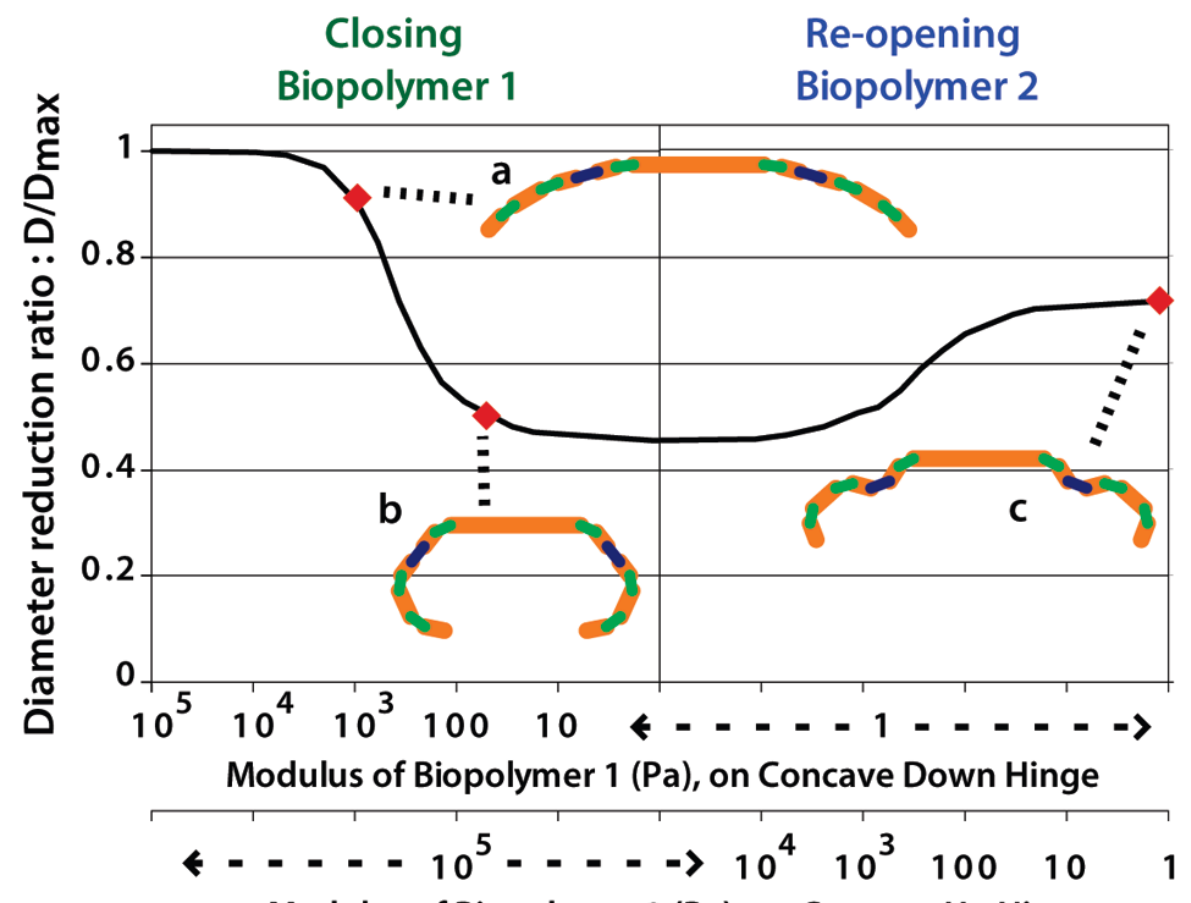

Modulus of Biopolymer $2(\mathrm{~Pa})$, on Concave Up Hinge

Key:

Concave Down Concave Up Rigid Hinge Hinge Segment

Figure T5. Diagram of the folding-unfolding path of enzymatically triggered two-way microgripper ${ }^{73}$ 


\section{Applications}

One of the most promising applications for stress-release based micro and nanostructures is the design of a new class of surgical instruments. In modern medicine there is an ever increasing trend towards miniaturization of the surgical devices. An emerging area of medical innovation involves the creation and utilization of tools that have no wires or tethers and are small enough to pass through various conduits in the body, such as the gastrointestinal tract, the reproductive or the circulatory system. The microgrippers, created by Gracias et al, represent an elegant approach towards the problem ${ }^{15}$.
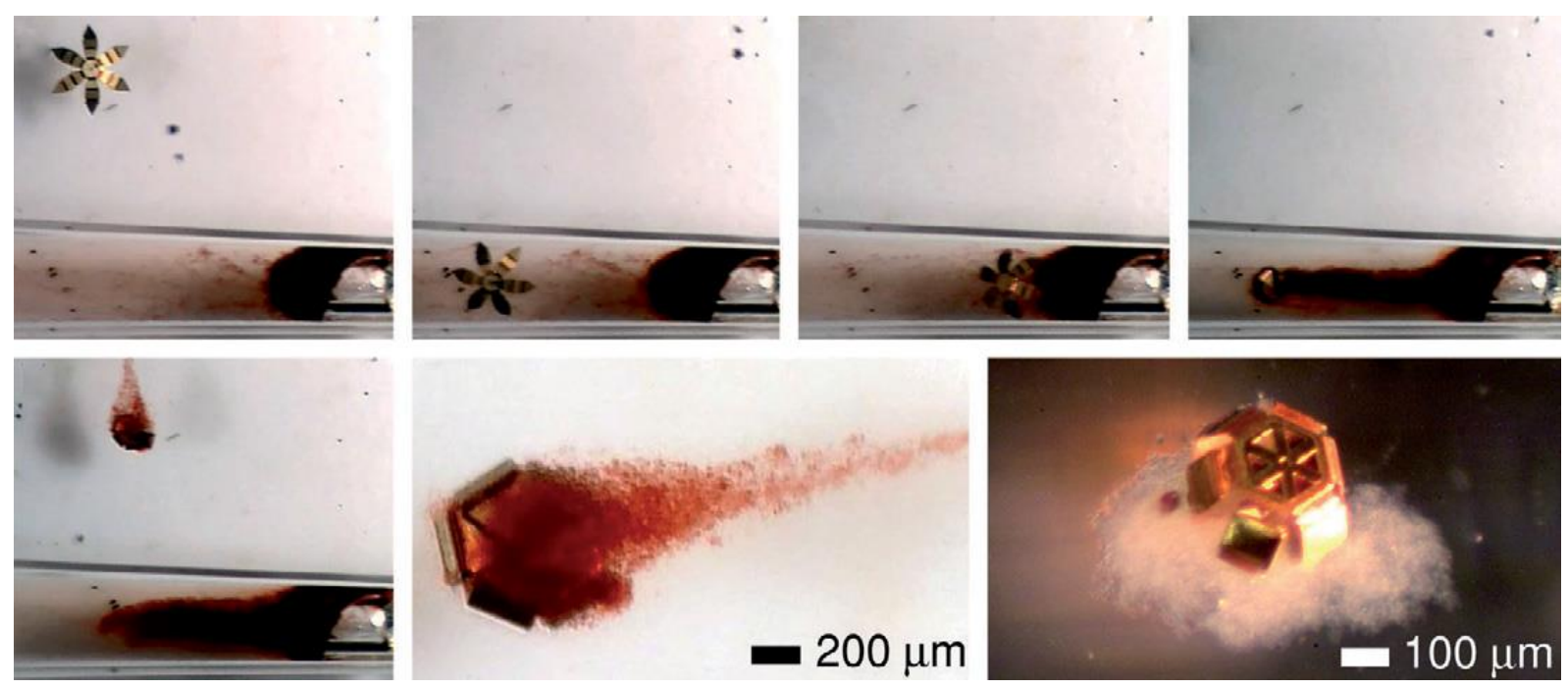

Figure T6. The microgripper is magnetically guided into a model blood vesicle (glass tube), then triggered to close and rip away a piece of blood clot ${ }^{15}$.

Because of their size, the microgrippers can freely swim inside the colon or some of the bigger arteries and their trajectory can be controlled by applying a magnetic field. They are mechanically robust enough to perform small incisions on soft tissues, providing one with the possibility to perform biopsy $^{74}$ or free blood vessels from clots $^{75}$ without the need for large openings, thus advancing the minimally invasive techniques to a new level. Recently, the possibility to integrate self-folded microstructures with an RFID tag was demonstrated ${ }^{76-78}$, granting a precise control over their location in the human body. 
Residual stress powered devices were also suggested as candidates for drug-delivery systems ${ }^{79}$.

\section{Lattice mismatch}

Folding due to lattice mismatch can also be classified as a stress-driven process. When a layer of material with a lattice constant $a_{L}$ is epitaxially grown on a substrate which has slightly different lattice constant $a_{S}$, accommodation of misfit along the interface is observed. If the misfit is small $(\leq 5 \%)$ and the interfacial bonding is strong, then all misfit is eliminated by elastic strain until the thickness of the film reaches a critical value. When this critical thickness is exceeded, it is energetically favorable for the part of the misfit to be accommodated by interfacial or misfit dislocations. If the critical thickness is not reached, the layer remains under stress ${ }^{80,81}$. This was first employed by Prinz et al to create self-rolling nanotubes on the example of InAs/GaAs bilayers ${ }^{82}$. The method is schematically illustrated on Figure T7.

The interatomic forces in the bilayer increase the interatomic distance in the compressed InAs layer and decrease it in the tensile-stressed GaAs layer. Therefore, the elastic forces $\mathrm{F}_{1}$ and $\mathrm{F}_{2}$ are oppositely directed, which results in a non-zero moment of forces $M$ that bends the layer and rolls it in a scroll. The diameter of folding depends on the

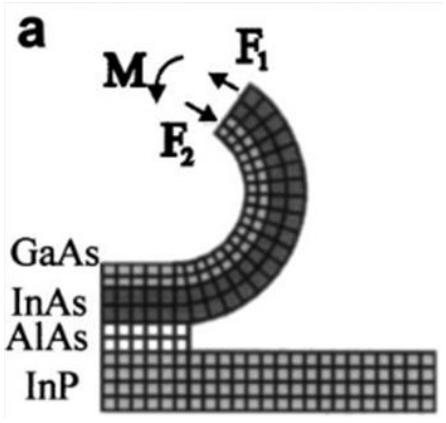

Figure T73. Schematic of stress-release rolling of a InAs/GaAs bilayer. Each cell represents an atom. value of the lattice mismatch $\Delta \mathrm{a} / \mathrm{a}$, as well as on the thickness $\mathrm{h}$ of the bilayers:

$$
D \approx h a / \Delta a
$$

The diameter of folding grows with the increase of the value of lattice mismatch and the thickness of the bilayer. Adjusting the lattice mismatch is a difficult task as one is forced to work with limited number of elements, whereas the thickness of the bilayer can be controlled with a precision down to the atomic scale. Self-rolled tubes 
with diameters ranging from $2 \mathrm{~nm}$ up to tens of microns have been produced with this method $83-85$.

\section{Applications}

The lattice mismatch approach was further developed by Schmidt et al to produce single- and double-rolled tubes based on various inorganic materials with manifold prospective applications. Self-rolled tubes based on inorganic materials sport sufficient mechanical stability to be incorporated into functional micro- and nanofluidic devices. They can serve as a model system to study the liquid behavior in round, smooth-walled, two dimensional confined, micro- and nanometer sized channels ${ }^{86}$. As the process of tube formation is based on the release of the material strain, the resulting structures are normally stress-free and are thus liable to improve the materials' tolerance against stress cracking. This makes them promising candidates for increasing the stability of energy storage devices such as lithium ion batteries. It was shown that lithium ion batteries based on $\mathrm{RuO}_{2} / \mathrm{C}$ self-wound nanomembranes as electrode materials exhibit enhanced cycling performance due to elastic strain minimization, homogeneous distribution of the active material among the structure as well as the composite micro/ nanohierarchical structure ${ }^{83}$. The preparation of hybrid $\mathrm{SiOx} / \mathrm{Si}$ microtubes that are thermally stable up to $850^{\circ} \mathrm{C}$ and serve as visible light emitters and ring resonators at the same time was demonstrated ${ }^{87}$. Selfrolling of $\mathrm{Au} / \mathrm{Ti} / \mathrm{InGaAs}$ and $\mathrm{Cr} / \mathrm{GaAs} / \mathrm{InGaAs}$ layers into a multifold stack consisting of polycrystalline metal and single crystalline semiconductor represents a novel way of producing superlattices ${ }^{88}$ suitable for spintronics ${ }^{89}$ and thermoelectric devices ${ }^{90}$. A very interesting utilization of the self-rolled microtubes is their use as self-propelled microjets ${ }^{91}$. In this particular design, the inner surface of the microtubes is covered with platinum. Platinum is a well-known catalyst that decomposes hydrogen peroxide into water and oxygen. Oxygen has only limited solubility in water and bubbles are formed inside the microtubes. As the bubble comes out, a reactive force acts upon the tube and propels it. 

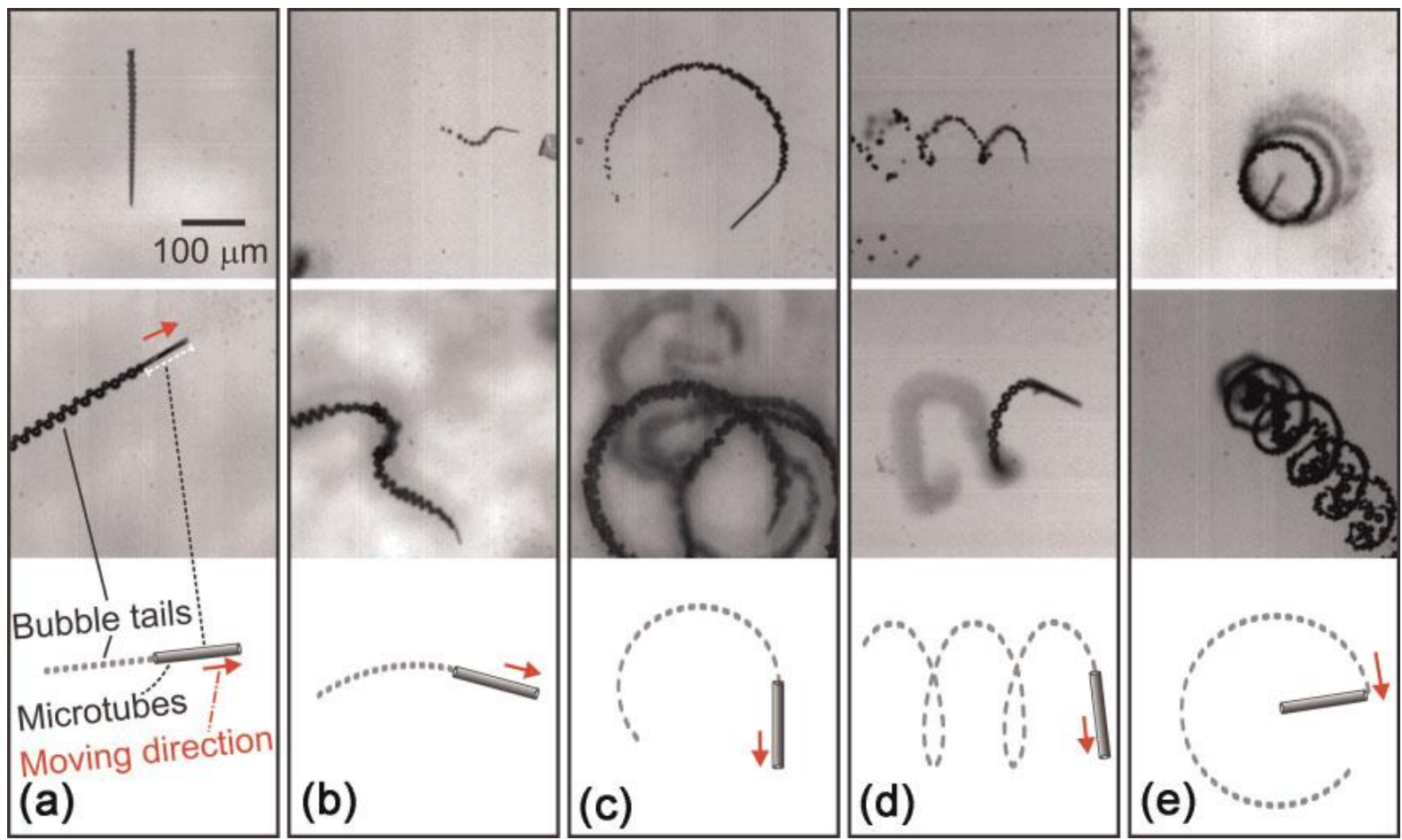

Figure T8. Different types of motion of the self-propelling microtubes. a) straight; b) zig-zag; c) circular; $d$-e) spiral.

By varying the shape of the opening, the swimming behavior of the tubes can be controlled. Introducing $\mathrm{Fe}$ into the tube makes it sensitive to a magnetic field and gives full control over the propelling direction ${ }^{28}$. These tubes can be used as microcarriers for the transportation of particles, cells or as drug-delivery tools ${ }^{92}$. They are also able to penetrate and kill cells and could be possibly used for a targeted destruction of tumors and harmful microorganisms ${ }^{84,85}$.

Other prospective applications include, for example, the SiGe:B/ Si:B based tubes, which can be used as thermoelectric gas sensors ${ }^{93}$, 94. The incorporation of ferromagnetic metals in the tubes' structure allows a remote control of the structures by magnetic field. Ni-Fe based microtubes are promising candidates for single-probe sensors or can be used simply as micromixers ${ }^{95}$.

\section{Surface tension as the driving force of folding}

At microscale, surface tension plays a predominant role in the interaction between liquids and solids and greatly surpasses other forces like 
gravity. For example, a thin liquid film on a lyophobic substrate will spontaneously break into spherical droplets; or, a thin solid film or a particle would float on the water-air interface. Surface forces are therefore a prominent and promising mean for fabrication at the micro- and nano-scales.

Droplets spontaneously adopt spherical form because a sphere exhibits the largest volume-to-surface ratio of all geometric forms, thus providing the greatest minimization of the surface tension. The force exerted in the process can be put to use for bending of thin films and creating 3D objects. This was employed in the work of Baroud et al ${ }^{96}$. Here, a water drop was deposited on a sufficiently thin PDMS sheet of a certain geometric shape. The size of the water droplet was comparable with the lateral dimension of the PDMS sheet; its volume ranged from 1 to $80 \mu \mathrm{L}$.
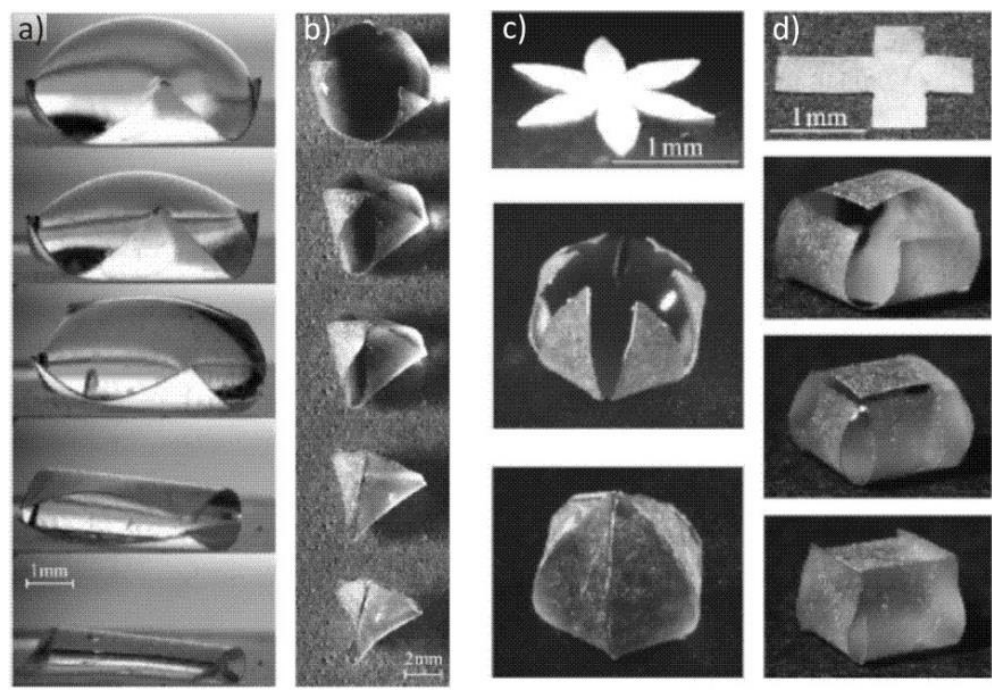

Figure T9. Folding of a thin PDMS film through surface forces. a) folding of a square film leads to the formation of tubes; b) triangular films result in a tetrahedron; c) the formation of near-spherical particles from a six-arm star; d) cube from a cross-like film ${ }^{96}$.

As it was mentioned above, the PDMS sheet stayed on the water-air interfaces due to capillary forces. That initially led to bending of its corners to enclose the drop. The water was then allowed to evaporate and as its volume decreased, the surface tension pulled the sheet around smaller volumes, thus increasing their curvature. The film eventually formed a 3D shape that encapsulated the drop. Furthermore, controlling the PDMS film geometry 
and thickness allowed to fabricate various 3D shapes - tubes, pyramids, spheres and cubes. ${ }^{96}$

\section{Applications}

A very promising application of surface tension regulated folding is the production of $3 \mathrm{D}$ electronic structures in the micrometer-to-millimeter range. This strategy was demonstrated for specifically designed millimeter-scale silicon objects, which were self-assembled into spherical and other 3D shapes, and integrated into fully functional light-trapping PV devices ${ }^{3}$. Spherical PV devices constructed from thin solar cells are particularly attractive, because they embed an intrinsic LT form factor ${ }^{97}$.
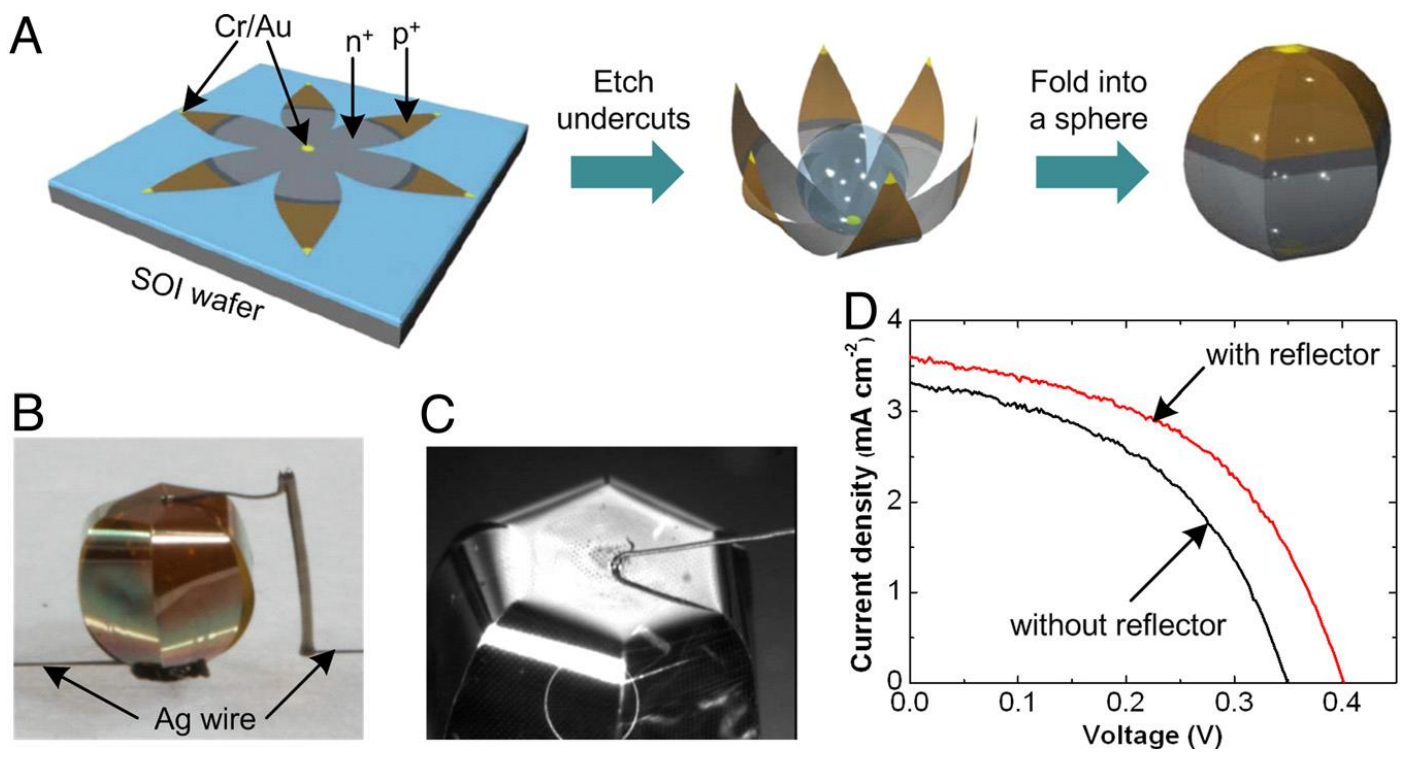

Figure T10. Miniature self-folded solar cell. a) illustration of the folding process; b) the already folded microsolar cell with sold wires; c) close-up of the soldering area; d) $A / V$ diagram for the microdevice ${ }^{97}$.

Soldering-driven self-assembly

Soldering is another approach that exploits surface tension to drive the self-assembly process. As it was already mentioned, two liquid droplets coalesce spontaneously when brought together. Gracias et al used that to create self-assembled microcontainers. Here, 2D metallic templates with solder hinges are produced by two-step photolithography. These templates further self-assembled into 3D hollow polyhedrons and surface tension of the molten solder provided the driving force for the process. When heated above 
the melting point of the solder, the hinges liquefied and coalesced. Ultimately, as the solder wetted the surface of the templates, coalescence of the hinges drove the parts together 98 .

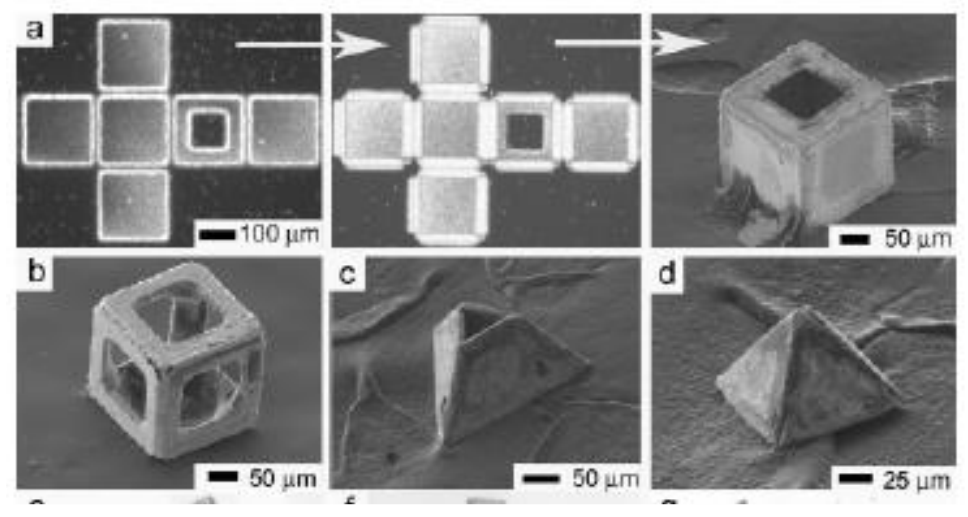

Figure T11. a) series of images, representing the soldering driven folding process; left: an overview of the initial structure; middle: after heating; right: SEM image of the folded cube; $b-d)$ different shapes obtained by soldering driven self-folding 98 .

Furthermore, this method can be utilized for the fabrication of microcontainers with several geometrical shapes like cubes and pyramids with a yield in the range of 60 to $90 \%$.

\section{Applications}

One field of applications of soldering-driven self-folding polymer thin films is the controlled encapsulation and release of drugs, particles and cells. Gracias et al demonstrated irreversible encapsulation of yeast cells inside self-folding SU8-PCl films upon heating above $60^{\circ} \mathrm{C} 99$. Porous microscaled cubes were used for sampling of nanoparticles and cells based on their size in small microliter volumes of liquid ${ }^{100}$. Porous structures were also used for an in vitro generation of $3 \mathrm{D}$ chemical patterns in stationary media for a variety of applications in chemistry ${ }^{2}$ and microbiology ${ }^{13}$. Self-folded objects with nanoporous walls and encapsulated cells were suggested as a prototype of artificial pancreas. Small molecules, such as glucose and dissolved oxygen, are able to pass through the pores while larger ones like antibody-proteins are sterically restricted from passing. This size-selective permeability of self- 
folded capsules bypasses the immune response - a highly demanded trait for organ and cells transplantation ${ }^{101}$.
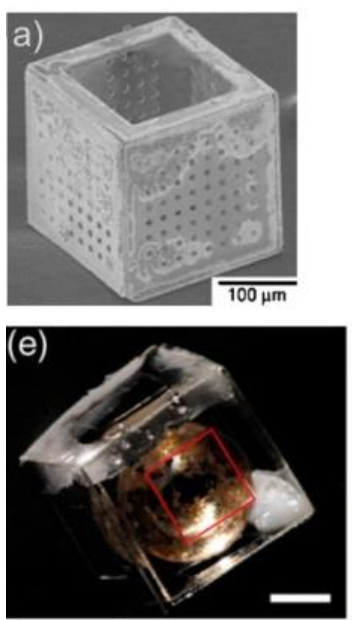
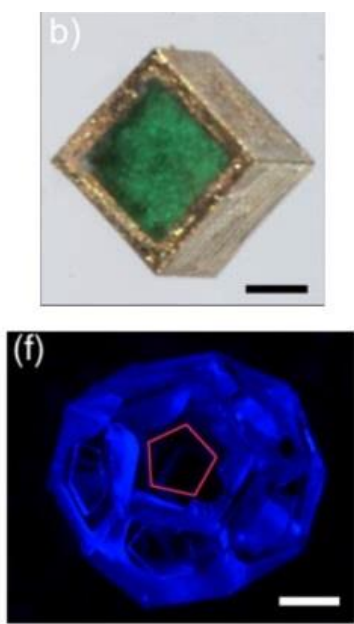
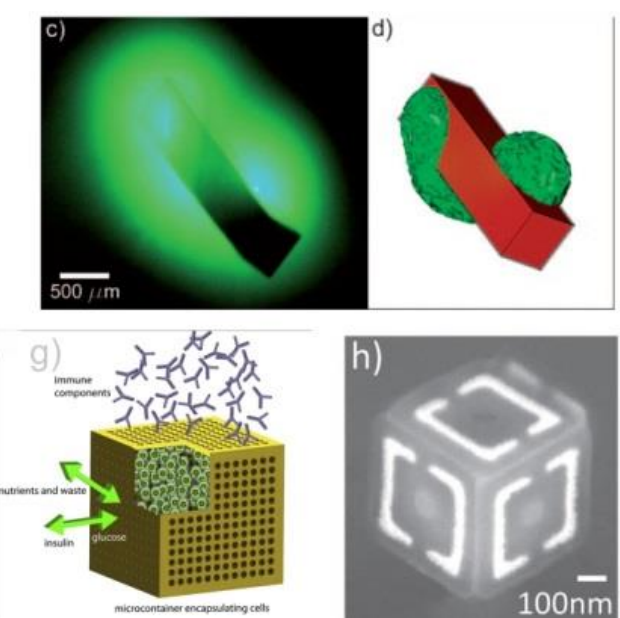

Figure T12. Examples of applications for soldering-driven self-folded devices. $a-b)$ SEM image of a porous cube and LM image of the same cube, filled with cell $\left.S^{13} ; c-d\right)$ patterned self-folded microcontainer for creating a controlled chemical environment ${ }^{102}$; e) microparticle encapsulated in a self-folded cube ${ }^{99}$; f) self-folded dodecahedron for encapsulation and transportation of cells and particles $\left.{ }^{103} ; g\right)$ porous cube as a prototype for artificial pancreas ${ }^{101}$; $h$ ) patterned microcube with enhanced optical functionality ${ }^{104}$.

Moreover, in biological applications patterns can function as chemical scaffolds for the study of important cellular functions in vitro ${ }^{102}$. Besides all the exciting biotechnological and medicinal perspectives these structures are apt to, they were also suggested as novel optical devices with split ring resonator patterns. It was shown, that a microcube with patterned sides sports an enhanced optical functionality ${ }^{104}$.

\section{Thermal actuation}

\section{Shrinking}

A simple and cost-effective system was proposed by Dickey et al. They used commercially available thin polystyrene films, known as Shrinky-Dinks, to create various $3 \mathrm{D}$ objects by temperature-induced self-folding. 

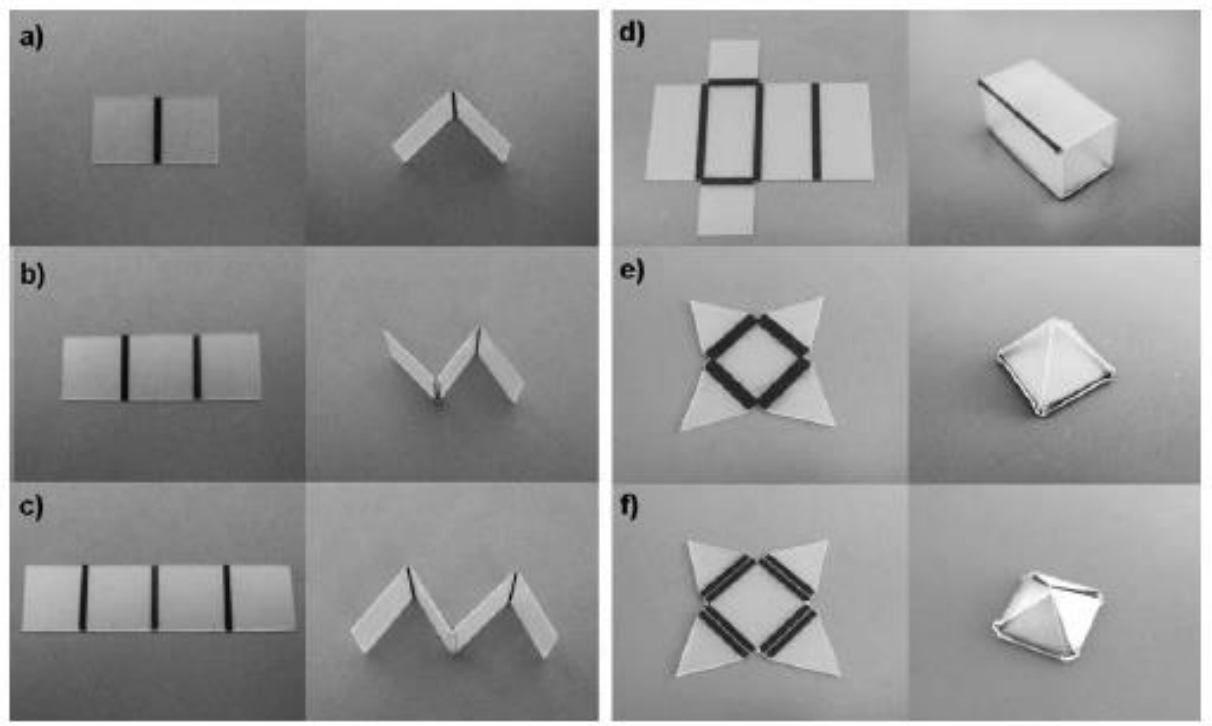

Figure T13. Examples of folding of differently patterned Shrinky-Dinks shapes $^{20}$.

Here, black hinges are printed on the surface of the polystyrene sheets by a desktop printer. The desired $2 \mathrm{D}$ shape was then manually cut off and illuminated with visible light. The polymer directly underneath the ink heated rapidly to exceed the glass transition temperature. As a result, the hinged regions relaxed and bent the sheet. The direction of folding (towards the light source or away from it) could be controlled by patterning either the top or the bottom side of the sheet, respectively ${ }^{20}$.

\section{Expansion}

It has been known for more than a century that a bilayer, consisting of materials with different coefficients of thermal expansion bends when the temperature is changed. This phenomenon has been ever since vastly employed in the design of thermal switches. The mechanism is thoroughly described in the previous chapter (see "Folding of bilayers").

\section{Applications}

Kalaitzidou demonstrated reversible adsorption-desorption of fluorescently labelled polyethyleneglycol inside PDMS-gold tubes at 60$70^{\circ} \mathrm{C}^{105}$. Recently, the thermal expansion of polymers was used to create a walking silicon microrobot. The actuation here is based on the thermal 
expansion of polyimide deposited in $\mathrm{V}$-shaped grooves etched in silicon with integrated heaters.

\section{Shape Memory Polymers (SMPS)}

The scientific community has paid a lot of attention to shape-memory polymers (SMPs) over the last decade, making them an important class of actively moving materials. For instance, SMPs are capable of changing their shape in a predefined way upon the application of an external stimulus. There are numerous SMP-based systems described in literature, varying both in their chemical composition as well as the stimulus they are sensitive to. The common conventional SMP systems include cross-linked polyethylene and Pe/nylon6 graft copolymers, styrene-based polymers, acrylate-based polymers, polynorbornene, epoxy-based polymers, thio-ene-based polymers. SMPs have been reported to be thermal-induced, light-induced, electro-active, water/moisture/solvent induced, $\mathrm{pH}$ sensitive and magnetic sensitive, based on their triggering stimulus. Nevertheless, ss diverse as SMPs are in terms of composition and functionality, they all are based, or can be described by, a relatively simple 3D architecture proposed by $\mathrm{Hu}$ and Chen ${ }^{106}$.

In this model, SMPs consist of a switch unit and net-points. The netpoints determine the permanent shape and are formed by chemical or physical cross-links with an interpenetrated or interlocked supramolecular

complex. The switch unit is responsible for controlling the shape fixity and recovery upon a specific and predetermined external stimulus. The driving force for strain recovery in SMPs is the entropic elasticity of the polymer network. 


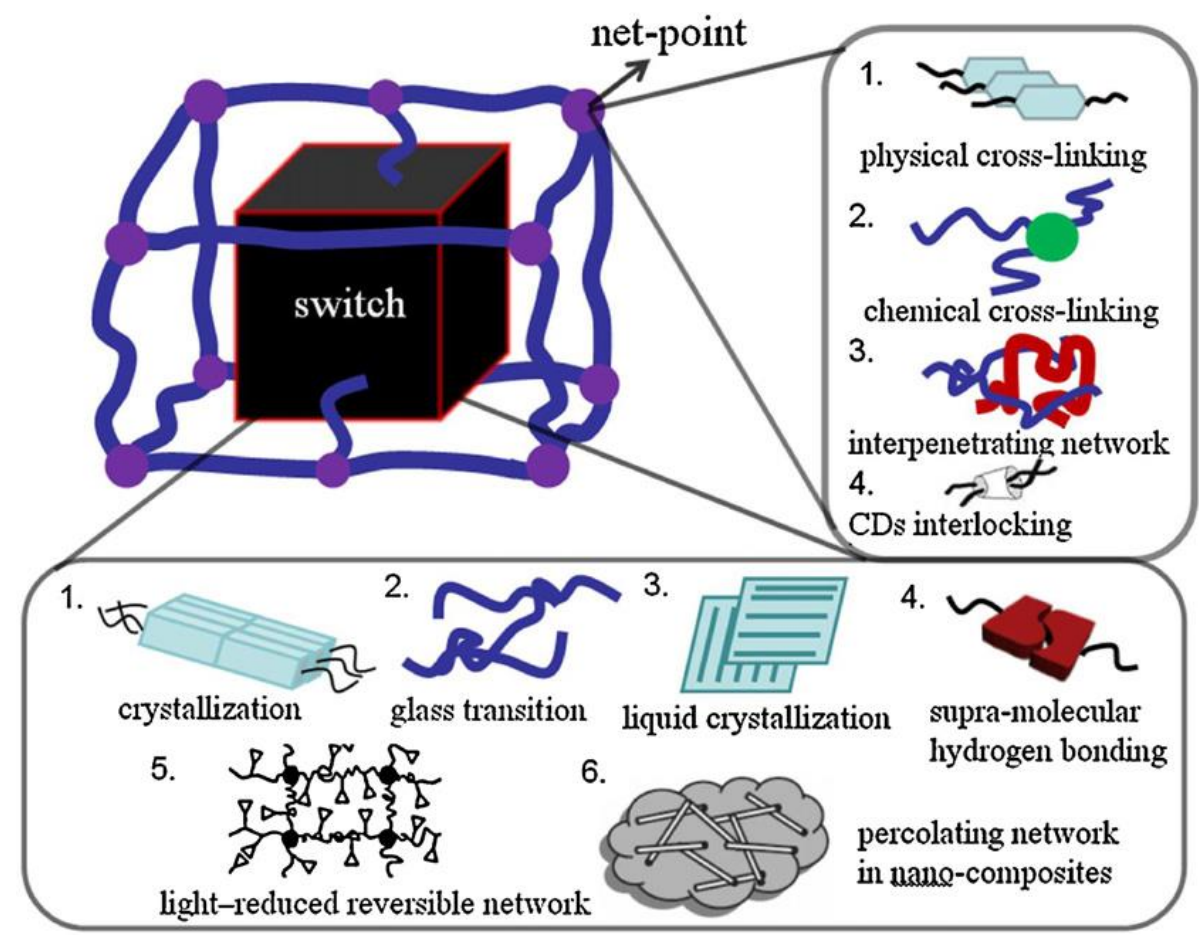

Figure T14. Illustration of possible basic building elements of $S M P_{S^{106}}$.

Additionally, the shape memory capability requires the deformation of the material into a temporary shape by application of external stress before. In most cases the stimulus, which is required for the actuation of the SMP system, needs to be applied during programming as well. Termination of the stimulus fixes the temporary shapes. When the stimulus is applied again, the SMP reverses the pre-defined pathway applied during the programming.

\section{Applications}

From all the stimuli-responsive actuating systems, SMPs are probably the most widely utilized material for construction of different devices for biomedical needs ${ }^{18,107}$, building of mechanical tools ${ }^{18}$, development of selfhealing materials ${ }^{108}$ and textile industry ${ }^{106,}$, 109. Some polymers are highly biocompatible, which makes them a perfect fabric for medical appliances. Employing this property, Jung and Cho reported their work on orthodontic wires made of shape-memory polyurethanes admixed with $\mathrm{PCl}$, as an alternative to the standard metallic ones. The SMP orthodontic wires possessed many advantages, such as low density, high shape recovery, easy processing, transparency and aesthetic appearance ${ }^{110}$. Furthermore, 
Rousseau et al invented SMP-based medical casts. The low density of polymers, their X-ray transparency, low cost and ease of processing provides obvious advantages compared to the traditional gypsum casts. SMP foams exhibit high shape recovery ratio and compression ability (up to 90\%) and can be potentially used for micro-foldable vehicles, micro-tags and hearing aids ${ }^{110-}$ 112. Present-day deployable dialysis needles, coils and neuronal electrodes are also made of SMPs ${ }^{113,114}$. Apart from medicine, the most well-known function of SMPs is as packaging materials.

\section{Polymers integrated with bio-actuators}

In order to move, cells have to exert a special kind of force, called the cell traction force (CTF), through surface receptors bound to extracellular matrix molecules coupled to rigid structures within the cell. This force is generated by actomyosin and actin polymerization and is directed toward the center of the cell body. Force can be generated because of the ability of adhesion receptors, such as integrins, to bind simultaneously to matrix components through their extracellular domains, and to the cytoskeleton (CSK) through their cytoplasmic domains. In this way, the adhesion receptors transmit the mechanical tension generated in the CSK to the cell's environment. Depending on the rigidity of the interaction substrate, this force can result either in cell movement, or it can deform the substrate. For example, PDMS micropillars sport a low enough stiffness for the traction force of a cell to bend them, and arrays of posts were used to investigate the contractile forces in smooth muscle cells and fibroblasts ${ }^{115}$. The CTF is normally in the range of nanonewtons ${ }^{116}$ and can thus be employed for manipulation of microobjects. This was only recently realized by Takeuchi et al. They used the CTF to fold 2D microstructures by patterning cells across microplates and then detaching the microplates from the glass substrate ${ }^{117}$. 


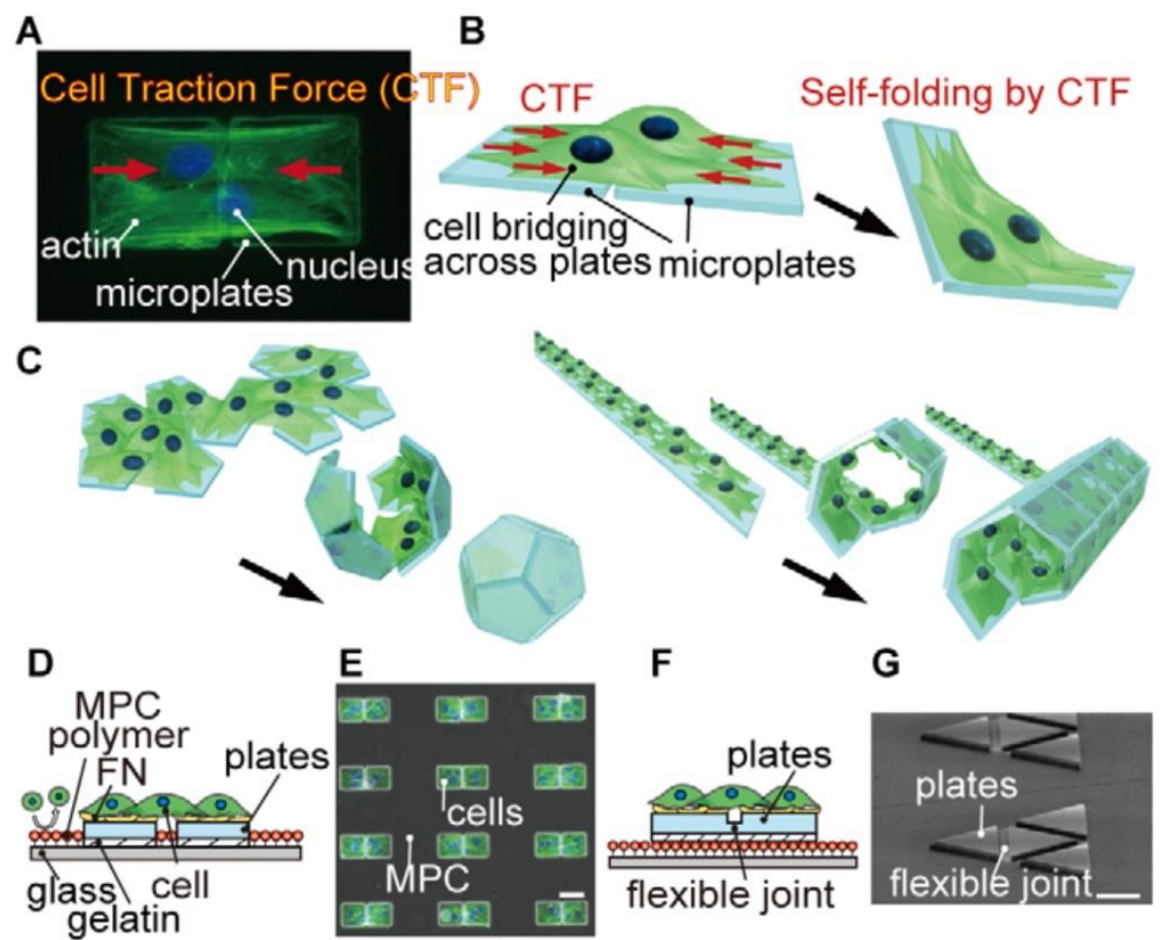

Figure T45. (A) The cells adhere and stretch across two microplates, and $C T F$ are generated toward the center of the cell body. Green and blue colors show actin and nucleus, respectively. ( $B$ and $C$ ) Schematic image of the cell origami: (B) the cells are cultured on micro-fabricated parylene microplates. The plates are self-folded by CTF. (C) Various $3 D$ cell-laden microstructures can be produced by changing the geometry of the plates ${ }^{117}$.

First, cells are cultured on the microplates. After the cells are patterned, they stretch across two microplates by extending their filopodia, thus connecting them, and CTFs are generated toward the center of the cell body. Since the CTF acts only on the upper surfaces of the plates, a rotational movement is generated ot the contacted upper edge of the microplate, lifting it off from the glass substrate. Noteworthy, CTFs exerted by multiple cells that contact with each other are directed centripetally at the cells' periphery, and no large traction stresses are executed under sites of cell-cell contact. Several factors govern the folding process, namely the distance between the microplates, the thickness of the sacrificial layer, the number of cells and the stiffness of the joint between the microplates.

The CTF vector always faces the center of the cell, i.e. the direction of the force is independent of the cell's position and orientation. Some cells, e.g. muscle cells, are capable of exerting force in a specific direction. Muscle cells 
contain protein filaments that slide past one another, producing a contraction that changes both the length and the shape of the cell. Muscles function to produce force and enable living organisms to move. The muscle-driven locomotion has served as an inspiration for the design of numerous machines and devices, where the role of the muscle is overtaken by a motor, and fibers serve as tendons. On the other hand, a different approach was introduced by Parker et al118. Instead of trying to mimic muscle functionality through complex machinery, they integrated myocardial tissue with polymeric thin films to create self-folding and moving structures. In their approach, cardiomyocytes were seeded onto a PDMS layer and the desired shapes were manually cut by scalpel. Bending or folding of the cut-off pieces occurred due to the contraction of the myocytes during the systole. By varying the thickness of the PDMS layer and the orientation of the muscle fibers, different folding scenarios were observed. The thickness of the PDMS layer had a strong influence on the form of the film during diastole. Because the elastic modulus of PDMS is much greater than that for cardiomyocytes (1.5 MPa versus $30 \mathrm{kPa}$ ), the stiffness of the muscular thin films (MTFs) was dominated by the polymer.

A

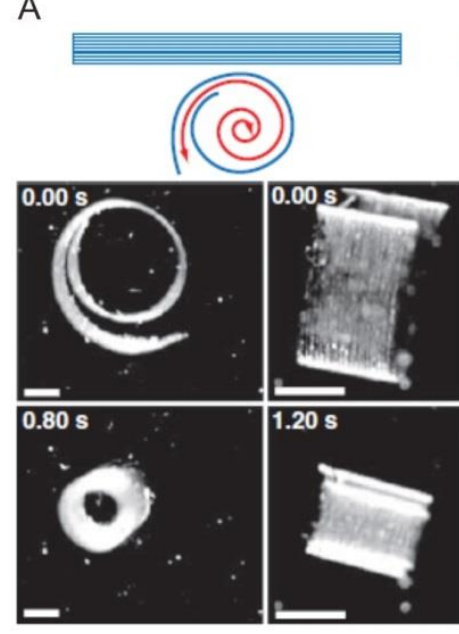

B

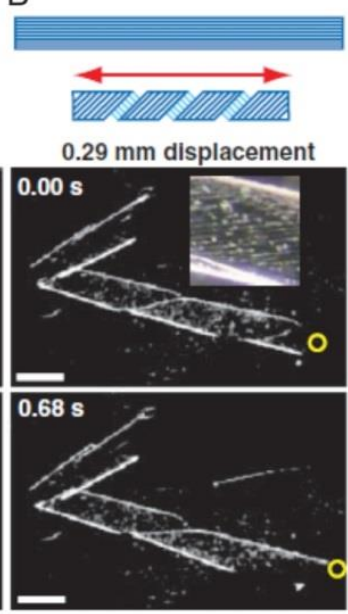

C

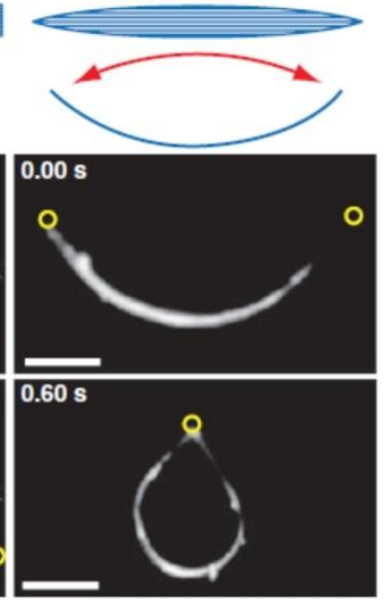

Figure T16. Folding of flat (a and c) and convex (e)PDMS strips with differently oriented muscle cells ${ }^{118}$.

MTFs thicker than $25 \mu \mathrm{m}$ remained planar during diastole, while thinner films adopted a curved conformation as soon as they were released from the substrate, thus defining two possible modes of systolic bending, 
flexion, or extension. Whether the cells were on the convex or concave surface was controlled by the curing temperature of the PDMS.

When MTFs with myocardin on the concave surface contracted, the radius of film curvature decreased, bending the film further out of plane. In this configuration, the MTF changed between a loosely rolled state during the diastole to a tightly rolled state during the systole. If the cardiomyocytes were placed on the convex surface, the radius of film curvature increased during contraction, bending the film back in plane (extension). Moreover, the direction of folding was governed by the alignment of the cardiomyocytes on the surface of the MTFs. Alignment along one of the sides of the rectangle films lead to folding in a perpendicular direction and thus tubes were formed (Figure T16a). Orienting the fibers $5^{\circ}$ to $15^{\circ}$ degrees off-axis resulted in a spiral-like folding, creating the structures on Figure T16b.

\section{Applications}

One of the obvious applications for the bio-integrated polymer films is the design of self-folding structures for cell culturing and tissue engineering. Furthermore, because cells are necessary for the folding process, the final structures are already cell laden and no complicated and potentially destructive loading techniques are required.

Additionally, soft robotic gripper and a myopod capable of autonomous or remote-controlled walking were created by integrating muscle cells with PDMS thin films (Figure T16c). ${ }^{118}$

\section{Swelling}

\section{Basics. Swelling in nature}

Swelling is an imprescriptible part of the dissolution process of polymers. The swelling of a polymer can wield pressures as high as several $\mathrm{MPa}$ and a significant amount of force can be exerted during the process. Swelling can thus cause major problems in some industrial facilities, e.g. during grain storage. It was shown that the swelling pressure of an air-dried corn seed can be as large as 4000 bars, making it a potential (and practical, if 
no preventative measures are taken) threat for the storage facilities ${ }^{119}$. On the other hand, swelling is a major course of movement in the plant kingdom. Plants lack muscles and the only way for them to change their position in space in a reasonably short time is by swelling (or drying) induced motion. The pine cone is a well-known natural actuator consisting of a central stiff rod and a number of scales starting from it. These scales hold and protect the seeds until they are ready to be released. Each scale consists of two types of tissues, one represented by cells with cellulose microfibrils, aligned parallel to the long side of the scale and the other - with perpendicularly placed ones. Upon drying the interfacial matrix between the fibers shrinks on the lower half of the scales. The presence of fibers leads to anisotropic contraction, which is hindered by the stiffer surrounding tissue, thus leading to a bending of the scales. Similar mechanism, which is also based on bilayer structure, is used by Aizoaceae to protect seeds against drought and to release them when the humidity is appropriate for them to grow. ${ }^{120}$ The folding and unfolding in both cases are relatively slow and occurs typically on the scale from several minutes to hours. However, despite the wide-spread belief that plants are only capable of slow motion, the folding of the Venus Flytrap (Figure T17,right) proves the contrary, as it is much quicker.

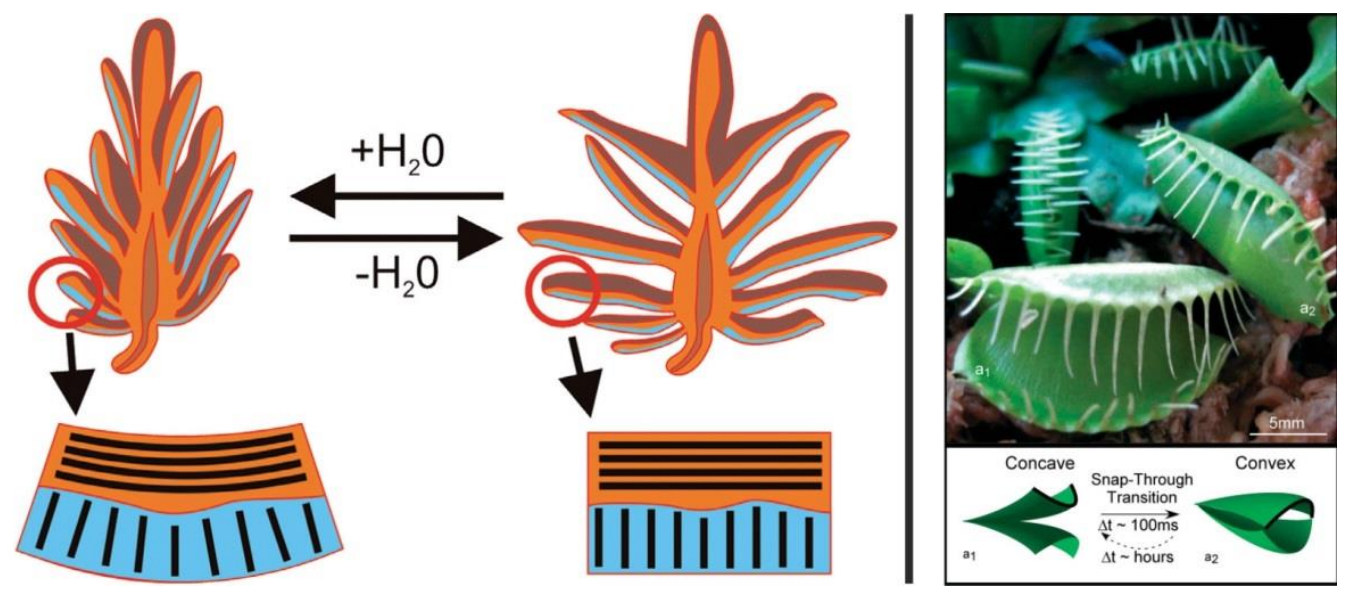

Figure T17. Examples of folding systems in nature: A sketch of actuation of scales in a pine cone upon drying and wetting (left)121. Snap-through of the Venus flytrap leaves from concave to convex occurs through the onset of an elastic instability of the films (right)122. 
With the plant closing its "jaws" in less than $100 \mathrm{~ms}$, it is one of the fastest movements in the plant kingdom ${ }^{123,124}$. In "open" state the leaves are doubly curved. Upon stimulation, the plant 'actively' changes one of its principal natural curvatures by hydration that results in snapping and closing of the "jaws". Similar mechanism is employed in the reflective pop bands for cyclers. Therefore, swelling can result in both slow and fast motions. As a consequence, swelling was proposed for the design of selffolding and actively-moving macro- and micro-devices.

\section{Swelling-based folding}

The folding mechanism in swelling-based structures somewhat resembles the bending of a bimetal film. In both cases, there are parts of the structure that expand to a greater degree than the rest of it, thus causing bending and folding. In contrast to metals, however, the swelling of polymers

normally produces significant volume changes (up to 10 times), while the thermal expansion of metals barely reaches several percent ${ }^{31}$. Moreover, the swelling degree of a polymer can easily be tuned by means of simple chemical modification, producing a control of the thickness of the final structure unmet in other systems ${ }^{32}$.

\section{Stimuli-responsive self-folding films}

Chemical modification of polymers enables one to have a precise control over the degree of swelling, but it also grants a command over their stimuliresponsiveness. The use of polymers that are sensitive to different signals allows the design of self-folding films upon immersion in solvent, change of $\mathrm{pH}$, temperature, electric or biochemical signals.

pH-responsive

Self-folding films sensitive to $\mathrm{pH}$ are commonly designed using weak polyelectrolytes as active polymers 60, 125-132. It was demonstrated that polystyrene-poly(4-vinyl pyridine) bilayer 125 as well as polystyrene-poly(4vinylpyridine)-polydimethylsiloxane trilayer 126 are able to roll at low $\mathrm{pH}$ when poly(4-vinylpyridine) is protonated and swells in water. The use of 
layers with a two-dimensional gradient of thickness allows thorough investigation of the folding process ${ }^{127}$. It was found that the rate of rolling increased with the acidity of the solution. Tube diameter and rate of rolling decreased with the increase of the UV exposure time. Moreover, an increase in the thickness of PS resulted in an increase of the tube diameter.

Lee et al. used $\mathrm{pH}$ sensitive poly(methacrylic acid) - $\operatorname{poly}(2$ hydroxyethyl methacrylate) 128 and PMAA/ PEGDMA ${ }^{129}$ patterned bilayer which folds when in contact with biological fluids. It was not shown that the folding depended on $\mathrm{pH}$; however, the systems were expected to respond to the $\mathrm{pH}$ signal since a weak polyelectrolyte (PMAA) was used. Gracias et al. fabricated millimeter large polyethylene glycol / poly-(N-isopropylacrylamide - acrylic acid) bilayers which were able to snap in response to the $\mathrm{pH}$ signal ${ }^{130}$. Moreover, one can also expect that this system is thermoresponsive. Huck et al. reported pH-responsive gold-poly(methacryloxyethyl trimethylammonium chloride) brush patterned films which fold in response to changes in $\mathrm{pH}$ and salt concentration 131. Very recently, Yang et al. demonstrated folding of $\mathrm{pH}$-responsive poly(2-hydroxyethyl methacrylate-coacrylic acid)/ poly(2-hydroxyethyl methacrylate) bilayers ${ }^{10}$.

\section{Thermoresponsive}

Thermoresponsive self-folding films can be designed using continuous thermal expansion, melting, shape-memory transition or polymers which demonstrate LCST (Lower Critical Solution Temperature) behavior in solutions. Kalaitzidou et al. used continuous volume expansion with temperature and demonstrated thermoresponsive rolling-unrolling of polydimethylsiloxane - gold bilayer tubes at $60^{\circ} \mathrm{C}-70^{\circ} \mathrm{C} 105,133$, which was due to different temperature expansion coefficients.

Gracias et al. used melting of a polymer, which then formed a droplet and forced patterned polymer films to fold. This was demonstrated on the example of patterned SU-8 photoresist - polycaprolactone film, which irreversibly folds at $60^{\circ} \mathrm{C} 134$ due to the melting of $\mathrm{PCl}$. In order to reduce the transition temperature and make the film more suitable for bio-related applications, Gracais et al. used photoresist hinges which are sensitive to 
temperature around $40^{\circ} \mathrm{C} 7,15,19$. The metal-polymer grippers irreversibly folded in response to temperature as well.

Lendlein et al. demonstrated new possibilities to design thermoresponsive macroscopic self-folding objects using shape-memory polymers based on different $\mathrm{PCls}^{18}$. At low temperatures, the structures are in a temporary shape. The films recover their permanent shape and irreversibly fold when heated, which could be accompanied by a change in transparency. The exact size of the self-folding film as well as transition temperature was not given.

Polymer bilayers, where thermoresponsive poly-(Nisopropylacrylamide)-based copolymers are the active component, are more suitable for encapsulation of cells. In aqueous media, PNIPAM-based hydrogels reversibly swell and shrink below and above $33^{\circ} \mathrm{C}$. Moreover, the temperature of transition between swollen and shrunk states can be tuned by a proper selection of the composition of the copolymer. As a result, PNIPAM$\mathrm{PCl}$ patterned bilayers fold and unfold, forming tubes of capsules below and above this temperature, respectively. ${ }^{5,135}$

\section{Solvent responsive}

In most cases, solvent-responsive self-folding films fold upon immersion in aqueous media. Such films contain water-swellable uncharged polymers. Lee fabricated partially biodegradable polyvinyl alcohol-chitosan ${ }^{9}$ and chitosan-poly(PEGMA-co-PEGDMA) bilayers 129 which folded in water due to swelling of polyvinyl alcohol and polyethyleneglycol, respectively. Jeong and Jang et al. developed an approach for the fabrication of millimeter size selffolding objects that are able to fold and form different 3D objects, such as tubes, cubes, pyramids and helixes ${ }^{136}$. For these purposes, water-swellable polydimethylsiloxane- polyurethane/2-hydroxyethyl methacrylate complex bilayers and patterned films were used. Since poly(vinyl alcohol), polyethyleneglycol and poly (2-hydroxyethyl methacrylate) and are not polyelectrolytes, swelling is expected to be independent from $\mathrm{pH}$ changes of the aqueous media. Thus, these systems immediately fold upon immersion in aqueous media that hampers loading of cells. Huck reported an example 
system that folds in methanol. This system was based on poly(glycidyl methacrylate) brush layer grafted to gold patterned films 131 .

\section{Light-responsive}

Light-responsive self-folding polymer films have been designed from thermoresponsive polymers with incorporated particles, which convert light into heat 20,137. Light-to-heat converting substance could be carbon nanotubes or any other black material. For instance, poly(N-isopropylacrylamide) hydrogel and pre-stressed polystyrene sheets (Shrinky-Dinks) were used as thermoresponsive components. The first system is active in aqueous medium, while the second one is capable of folding in both air and water.

\section{Other systems}

Except for $\mathrm{pH}^{-}$, thermo- and solvent-responsive systems, there are also several examples of systems, which fold in response to other stimuli such as presence of enzymes or applied electric field. Smella ${ }^{27}$ and Jager ${ }^{14}$ et al., who first introduced the self-folding films, demonstrated folding and unfolding of a patterned gold film with polypyrole hinges in a response to an electric signal. As discussed previously, Whilesides et al. fabricated electro-responsive selffolding bilayers, which consisted of poly(dimethylsiloxane) with aligned cardiomyocytes 118. The polymer-cell film adopted functional threedimensional conformations when an electric signal was applied. Along with that, these centimeter-scale constructs can perform functions as diverse as gripping, pumping, walking, and swimming with fine spatial and temporal control.

As it was already mentioned, enzyme-sensitive self-folding films were developed for the first time by Gracias et al. The approach is based on the use of self-folding metallic grippers with active polymer hinges, which are sensitive to the presence of enzymes ${ }^{73}$. Two kinds of biodegradable polymers were used. The gripper, which is unfolded in its initial state, folds when the first polymer is degraded due to the addition of the first enzyme. The gripper unfolds when the second enzyme is added and the second polymer is degraded. As a result, a full circle of folding and unfolding is achieved. 


\section{Fabrication of Polymer Films}

Overall, there are two main approaches which utilize swelling as a driving force for the design of self-folding polymer films. One of them comprises structures which fold and bend in a prescribed manner due to their intrinsic inhomogeneities like hinges or an inherent chemical gradient. On the other hand, the second approach is based on homogeneous hinge-free polymer-polymer or polymer-metal bilayers. In either way, folding occurs due to the difference in swelling of particular sections of the structures. Therefore, the swelling-based actuation methods can be classified in two major groups: non-homogeneous and homogeneous. Obviously, the fabrication processes for those two methods have to be quite different, as they are bound to introduce fundamentally different properties in the films.

\section{Non-homogeneous swelling}

Non-homogeneous swelling is described by a gradient in the solvent concentration in the bulk of the material after completion of the swelling process. Non-homogeneous swelling results in different volume changes in the structures and can therefore be used to execute bending and folding.

As non-trivial the task of creating a layer with controlled inhomogeneity might seem, as a matter of fact, there exist plenty of methods to achieve that. Below, some of the most employed techniques are explained in details.

\section{Grayscale photolithography}

In its most basic implementation, the grayscale photolithography technique includes a series of photo-masks that are used in a certain sequence to define discrete regions of the film, each of them having a different homogeneous level of expansion. 

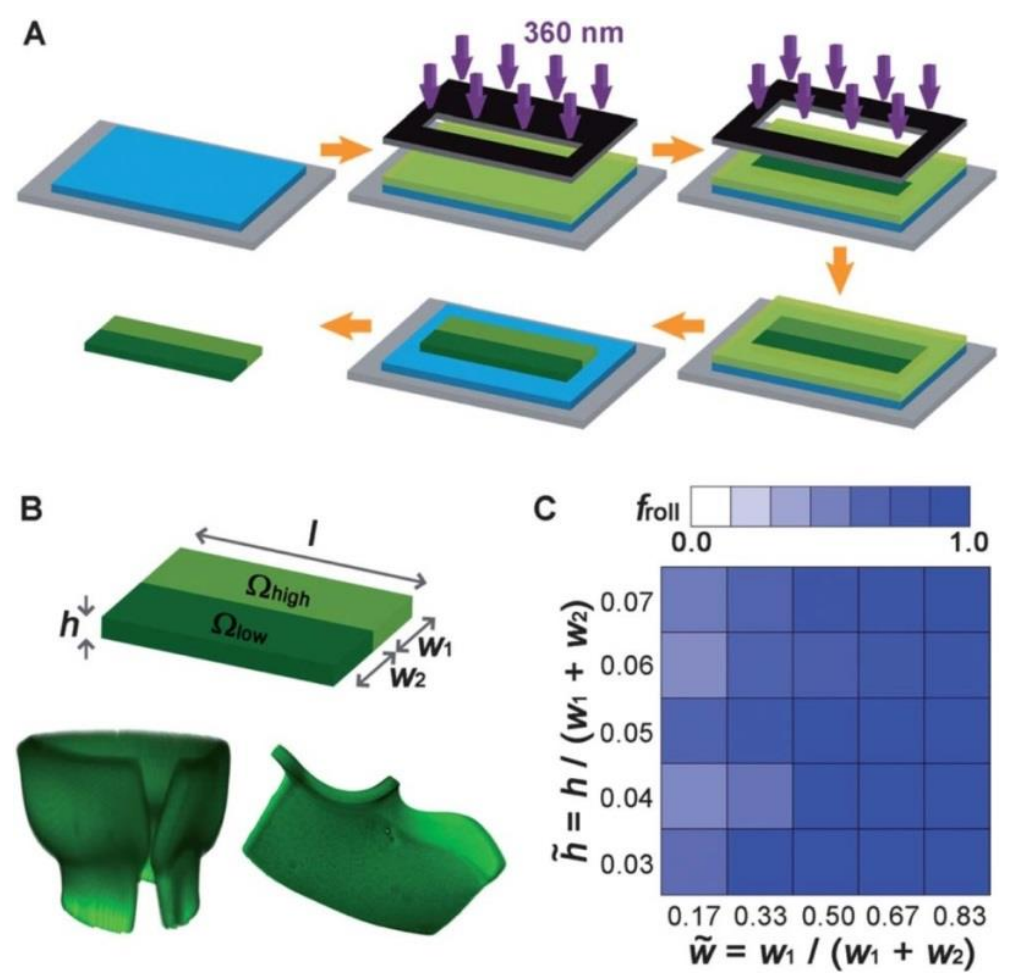

Figure T 18. Schematic illustration of the grayscale photolithography process ${ }^{138}$.

The process is schematically shown on Figure T18. A silicon substrate covered with a $100 \mathrm{~nm}$ thick sacrificial layer (blue) is coated with an 8-17 mm thick film of a photo-crosslinkable temperature-responsive copolymer. The film is then exposed to UV light through two aligned photomasks in sequence, yielding variations in the degree of crosslinking and therefore the degree of swelling (indicated by different shades of green). After crosslinking, the film is developed with a marginal solvent to dissolve uncrosslinked regions, and the patterned polymer film is released upon immersion in an aqueous medium $^{71}$. Moreover, parts with different degrees of crosslinking express individual intensity of swelling: the lower the degree of crosslinking, the stronger the hydrogel expands. Additionally, objects with multiple hinges and plates can be patterned by grayscale photolithography, further increasing the arsenal of attainable structures.

\section{Differential crosslinking}

The idea of differential crosslinking somewhat resembles the notion of grayscale photolithography. Here, a gradient in the degree of crosslinking of 
the polymer film is also created through controlled illumination. In contrast to grayscale photolithography, this gradient is generated throughout the depth of the film, while preserving homogeneity in the planar dimensions. The self-folding ability of such structures was shown on the example of differentially crosslinked star-like patterns ${ }^{139}$. For instance, heterogeneous SU-8 films with crosslink gradients along their thickness were created by acid-initiated cationic polymerization. The extent of crosslinking within the SU-8 film was varied by controlling the magnitude of ultraviolet exposure energy ${ }^{140-142}$.
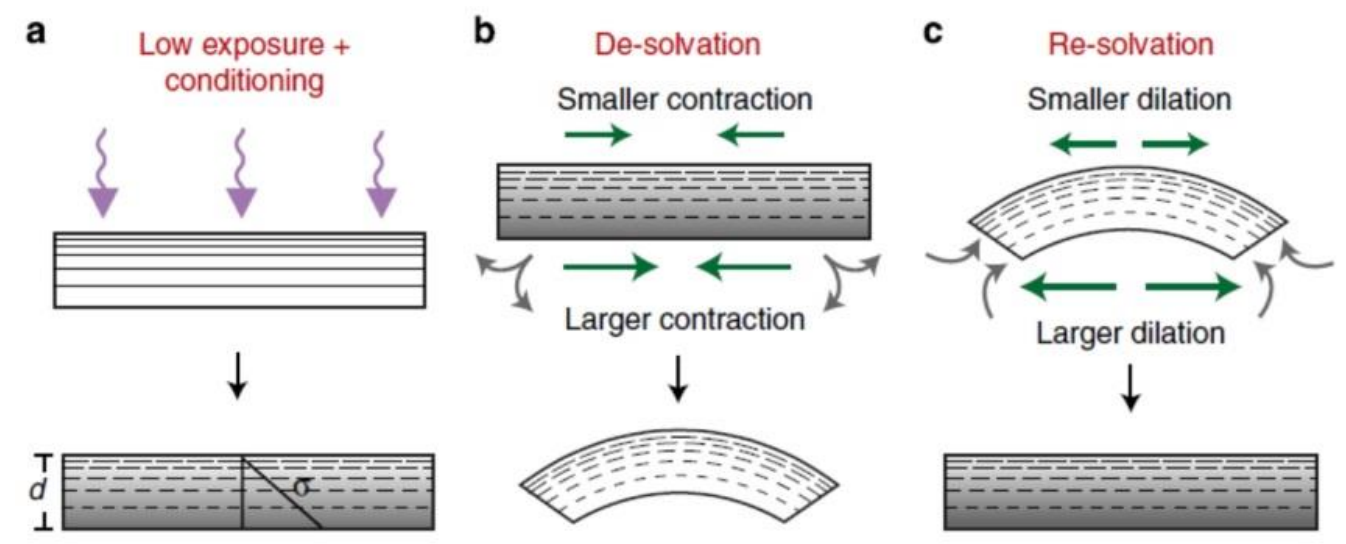

Figure T19. An illustration of the differential crossliknking process (a) and its bending mechanism ( $b$ and $c)^{140 .}$

After exposure and baking, the photopatterned films were developed, released from the substrate, and soaked in organic solvents such as acetone to condition the films and generate a stress gradient for self-assembly. The conditioning step removed residual uncrosslinked material. As a result, these differentially photo-crosslinked and acetone-conditioned SU-8 films then spontaneously and reversibly curved on de-solvation by drying or the addition of water, and flattened when re-solvated by organic solvents.

\section{Concentration gradient}

Amongst all hither described methods for achieving a nonhomogeneous swelling in a polymer film, the construction of hydrogels with internal lateral gradients in the monomer concentration is probably the most experimentally sophisticated technique. It was realized by Sharon et al on the 
example of circular and cylindrical PNIPAM hydrogels ${ }^{62}$. For this purpose, programmable actuated valves were used to inject solutions with gradients in monomer concentration into a mold (Hele-Shaw cell). The reaction parameters were controlled in a way that polymerization took place in less than a minute, freezing the imposed gradients within the gel. Consequently, the constructed sheets were flat below $33^{\circ} \mathrm{C}\left(33^{\circ} \mathrm{C}\right.$ is the LCST of PNIPAM) but were programmed to shrink differentially upon activation at higher temperatures.

\section{Thickness gradient}

In contrast to the concentration gradient, it is relatively easy and straightforward to create a thickness gradient in a polymer film. Jeong et al achieved that by simply conducting polymerization on a tilted substrate ${ }^{143}$. The folding ability of the prepared structures was checked on the example of PDMS-polyurethane/PHEMA system. Different solvents, such as hexane, acetic acid and ethyl acetate, were used for this purpose. PDMS doesn't swell in these solvents, whereas the polyurethane/PHEMA layer does, which leads to bending and folding of the bilayers. Hence, non-homogeneous folding in the prepared structures occurred due to the difference in the thickness ratio of the two polymers.

\section{Localized solvent deposition}

All of the above mentioned techniques make implement of structures with intrinsic, predetermined during preparation, inhomogeneities, which fold in a sophisticated manner when fully immersed in an appropriate solvent, i.e. in a homogeneous medium. On the contrary, Holmes et al took another path. In his work, non-homogeneous swelling was induced through localized exposure of PDMS films (slender beams and thin circles) to a favorable solvent. Despite the fact that curving and bending of the films was demonstrated, no real 3D forms were obtained by this method ${ }^{144}$.

\section{Homogeneous swelling}

Homogeneous swelling denotes that at the end of the process the solvent concentration in the bulk material is uniform. If a piece of polymer of 
any given shape undergoes homogeneous swelling, no bending or shape change will take place, as the expansion of the material is equal in all directions. For folding to occur, inhomogeneity has to be introduced along at least one swelling vector. This is done by introducing covalently bound nonswelling parts or layers to the structure, e.g. a metal or a polymer with different lyophilicity. This non-swelling layer restricts the expansion of the polymer and creates appropriate conditions for wrinkling, creasing and folding to occur.

Experimentally, polymer films with different shapes can be obtained either by cutting 105, 125, 133, using microwell-like substrates 9, 128, 129, photolithography $5,103,130,135$, or by grafting 63,131 . Cutting is a straightforward method that allows fabrication of millimeter-sized structures. Therefore, the main advantage of this method is its simplicity and applicability to almost all combinations of polymers. On the other hand, the use of microwell-like substrates is technically more complicated but it grants greater variety in terms of complexity of the design and downscaling of the composites. Further, photolithography of bilayers allows for large-scale fabrication of self-folding objects of different shape and size starting from several microns. In contrast, surface grafting comprises the advantage that all polymer chains are covalently bound to the substrate during preparation and no further crosslinking is necessary.

\section{Cutting}

The process of cutting, whether performed manually or by means of machines, is self-explanatory and won't be discussed in this work. A noteworthy example is, probably, the very recently demonstrated by Luchnikov et al. method for upscaling the fabrication of self-folding films by merely scratching polymer bilayers with an array of metallic blades ${ }^{145}$, which can be considered a kind of cutting.

\section{Photolithography}

Photolithography is one of the most readily employed microfabrication techniques and is used to create patterns into a material. The photolithographic process consists of a number of steps in which a desired 
pattern is generated on the surface of a substrate through exposure of regions of a light-sensitive material to ultraviolet (UV) light. Figure T20 summarizes the main steps followed in photolithography. In the first step, a substrate material, such as silicone or glass, is coated with a layer of a photoresist, or light-sensitive polymer.

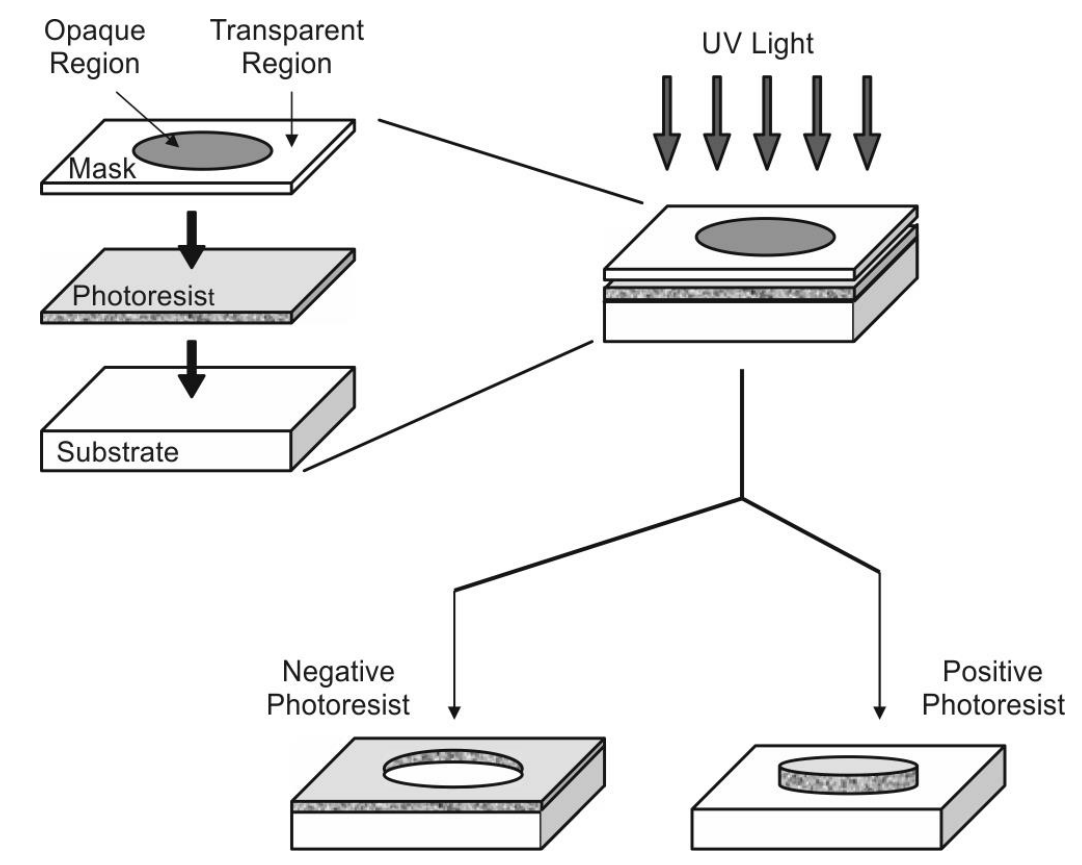

Figure T20. An illustration of negative (left) and positive (right) photolithography ${ }^{146}$

A photomask, made by patterning with an opaque material the desired shape on a glass dish or other transparent material, is placed on top of the substrate and photoresist. This assembly is then irradiated with UV light, thus exposing the sections of the photoresist not covered by the opaque regions of the photomask. Depending on the type of photoresist utilized, the photoresist polymer will undergo one of two possible transformations upon exposure to light. When light illuminates a positive photoresist, the exposed regions break down and become more soluble in a developing solution. As a result, the exposed photoresist can be removed when in contact with the developing solution. A negative photoresist, on the other hand, becomes crosslinked upon exposure to light, thus becoming insoluble in the developing solution. Consequently, upon contact with the developing solution, only the parts not exposed to light will be removed ${ }^{146,147}$. 
Therefore, photolithography can be used for the fabrication of patterned polymer films. This procedure, however, requires mask alignment and several steps of photolithography. On the other hand, it yields the broadest range of self-folding objects.

\section{Microwell technology}

The process of microwell preparation of polymer structures combines microtransfer molding ${ }^{148}$, discontinuous dewetting9, 149, and sacrificial layer methods. Microtransfer molding is performed by filling microwells on a PDMS mold with a liquid precursor, pressing the mold against the substrate, and solidifying the precursor. This method is often plagued with a thin residual film between the produced microfeatures. Furthermore, discontinuous dewetting is a technique used for filling a large number of microwells with a non-wetting liquid via the dewetting process of the liquid from the surface bearing the microwells. Using this technique, a liquid polymer precursor can be filled in the microwells but not around the surrounding area on a PDMS stamp. Additionally, to produce bilayer structures, two different liquid precursors can be filled and cross-linked in microwells ${ }^{129}$.

\section{Surface polymerization}

Another widely used technique for the preparation of thin polymer films on a surface is the grafting method. The most common grafting methods are "grafting to" and "grafting from"150. The "grafting from" technique involves polymerization that is initiated at the substrate surface by attached (usually covalently bonded) initiating groups. In contrast, the "grafting to" technique involves a reaction of (end-)functionalized polymer molecules with complementary functional groups located on the surface, resulting in the formation of tethered chains ${ }^{151}$.

Employing this approach, microcontact printing followed by brush growth ("grafting from"), crosslinking, and substrate etching was used for the preparation of polymer-covered thin Au microstructures ${ }^{131,152}$. Depending on their shape and layer thickness, the composites were shown to form various $3 \mathrm{D}$ constructions under appropriate stimulus. 


\section{Applications}

The broad applicability of polymer-based self-folding films in biological, biomedical and medical research and practice is mainly based on the easiness to tune their properties. Polymers are readily modified by simple chemical reactions to gain traits like biocompatibility, softness, the ability to respond to external stimuli. Therefore, there are plenty of reports describing the fabrication of self-folding films with different responsive properties. For example, there are $\mathrm{pH}$-responsive systems based on polyelecrolytes ${ }^{10,60,125,128-}$ 132, 153, 154; thermo-responsive based on gradient of thermal expansion 105, 133, melting of a polymer $997,15,19$, or on thermo-responsive polymers 5,135 ; solvent-responsive 129, 131, 143, 144; triggered by electric signal 2714118 or enzymes 73 ; light-sensitive based on light-to-heat conversion ${ }^{137}$.

Recently, biodegradable self-folding films made of commercially available biodegradable polymers have been extensively used to encapsulate cells (Figure T21b) ${ }^{6}$.

a)

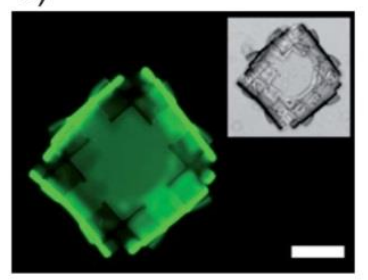

d)

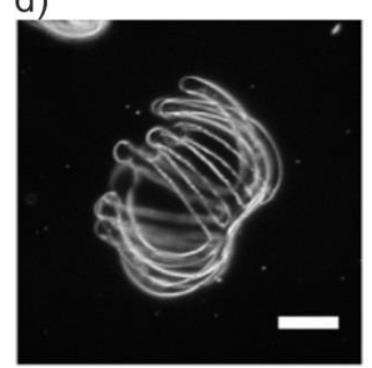

b)

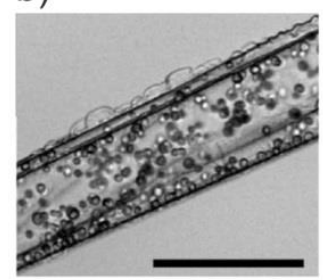

e)

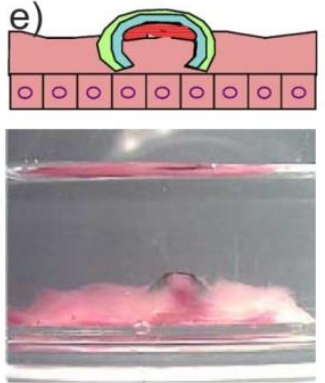

c)

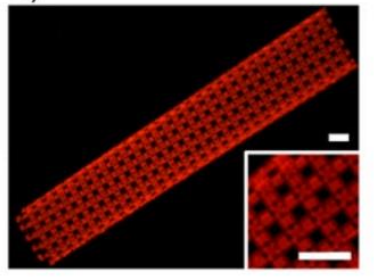

f)

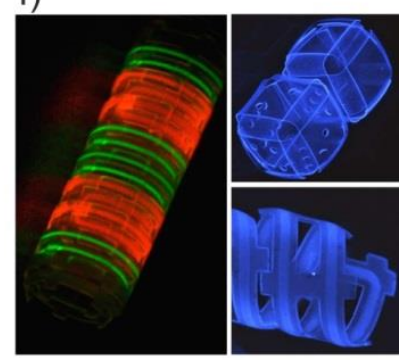

Figure T 21. Examples of applications of self-folding polymer films:, (a) -selffolded cube, produced by grayscale photolithography (b) - yeast cells encapsulation inside a fully biodegradable self-rolled film ${ }^{6},(d)$ - self-folded microstar for drug-encapsulation, (e) - smart plasters which direct diffusion of drugs and prevent their leakage ${ }^{128,}$ (c) and (f) - 3D mirofludic devices ${ }^{139}$.

Self-folding films can also be used as smart plasters (Figure T21e). Lee demonstrated this concept on the example of a millimeter size poly(methyl 
methacrylate) - poly(2-hydroxyethyl methacrylate) bilayer with attached mucoadhesive drug layer. The non-swelling PHEMA layer serves as a diffusion barrier, minimizing any drug leakage in the intestine. The resulting unidirectional release provides improved drug transport through the mucosal epithelium. The functionality of this device was successfully demonstrated in vitro in a porcine small intestine ${ }^{128}$.

There are several non-biorelated examples of application of self-folding polymer films. Deposition of a patterned metal on the polymer bilayer allowed fabrication of self-rolled tubes with a patterned conductive inner wall. ${ }^{125}$ In another example, pyrolysis of a poly(styrene)-poly(4 vinyl pyridine)poly(dimethylsiloxane) trilayer ${ }^{153}$ were used for fabrication of silica tubes. Gracias et al used self-folding polymer films to fabricate curved selfassembled microfluidic networks ${ }^{139}$.

\section{Complex Shapes}

As a matter of fact, the shape of a formed $3 \mathrm{D}$ object depends on the $2 \mathrm{D}$ shape of the polymer films. The simplest case of a self-folded object is a tube, formed from a rectangular bilayer (Figure T22a) 5, 9, 105, 125, 128, 129, 133. Envelope-like capsules with rounded corners or nearly spherical ones are formed from star-like polymer bilayers with four and six arms, respectively (Figure T22b,c) 9, 129. Helixes of different kinds are formed from polymer stripes with a gradually changing thickness ratio between the polymers ${ }^{136}$. In these examples, simple rounded figures based on different combinations of fully or semi-folded tubes are formed. Moreover, because of the isotropy of the mechanical properties of the bilayer, the formation of hinges during folding of the bilayers is considered improbable.

Objects with sharp edges are formed from patterned bilayers where the active component is deposited locally (Figure T22e-h). The active component can either swell/shrink or change its shape due to melting. Along these lines, cubes and pyramids are formed from a patterned bilayer with active junction elements ${ }^{103,130,136 .}$ 


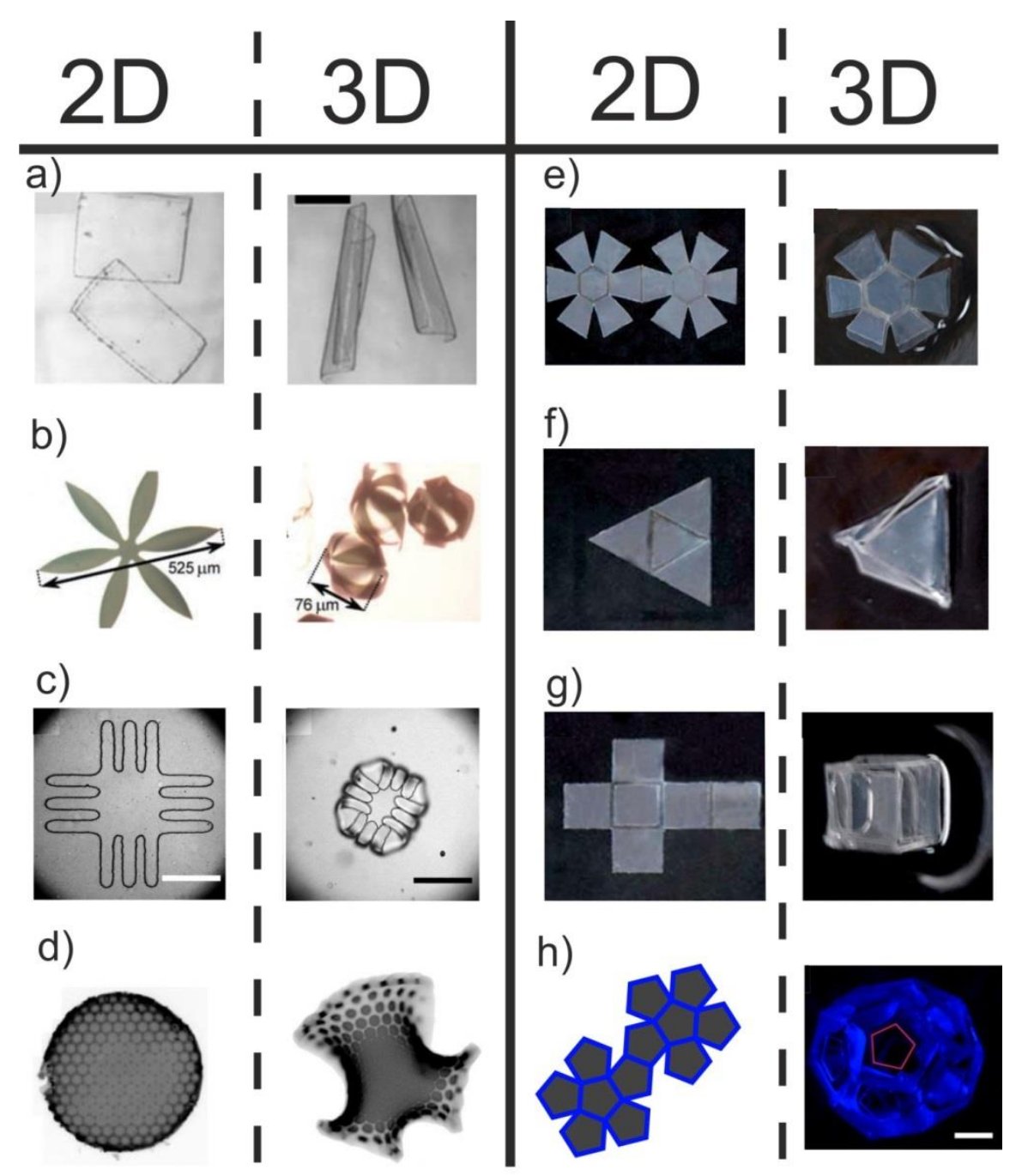

Figure T 22. Examples of self-folding polymer films with different shapes: tubes 105 (a), capsules 9,129 (b,c), saddle-like surface ${ }^{138}$ (d), flat ball 136 (e), pyramid ${ }^{136}(f)$, cube ${ }^{143}(f)$, dodecahedron ${ }^{103}$ (h).

For instance, Santangelo and Hayward reported on photopatterned hydrogel films that yield temperature-responsive gel sheets and can transform between a flat state and a prescribed three-dimensional shape. The hydrogel was patterned through a process, dubbed halftone gel lithography, by using only two photomasks, wherein highly cross-linked dots embedded in a lightly cross-linked matrix provided access to nearly continuous, and fully two-dimensional, patterns of swelling. This method is used to fabricate surfaces with constant Gaussian curvature (spherical caps, saddles, and cones) or zero mean curvature (Enneper's surfaces), as well as more complex and nearly closed shapes (Figure T22d). 
Importantly, in most of the cases folding runs in one step. Step-by-step folding of different elements of self-folding films can be achieved by local activation of selected areas by light ${ }^{155}$. Another possibility is to use two or more kinds of active material, which are sensitive to different stimulus. Gracias et al demonstrated two-step deformation of patterned films where the active elements are two kinds of biodegradable polymers. Each of these polymers is degraded by a specific enzyme. As a result, the films fold when the first enzyme is added and unfold when the second one is added. ${ }^{73}$ On the other hand, there are reports that folding in nature can have a very complex character, which strongly depends on the swelling path 156,157 and may result in a multi-step folding (development of curvature in different directions) 120.

Polyhedra represent an interesting group of self-folding microorigami. A common strategy for the design of these structures is the formation of a $2 \mathrm{D}$ layer, comprising of many connected together pieces that have the same geometrical shape and that form the sides of the polyhedron upon folding. There are, however, many different ways for connecting these structural elements and not all of them will give the desired object after assembly. For example, there exist many different ways to link the vertexes of 6 square pieces but only eleven of them can, at least theoretically, fold into a cube. If one is given a simple polygon (and its interior) in the plane, Alexandrov's theorem gives conditions under which this polygon can be folded by the identification of points of the polygon's boundary to a convex polyhedron or a double covering of a convex polygon. Despite its predictive powers, this theorem is not widely used for the design of self-folding polyhedra, because of its complexity, especially when external influences on the system have to be accounted for. Gracias et al took an experiment-based approach backed-up with some simplified calculations to solve the problem. In their approach, they considered polygons that have fold lines (also dubbed as internal hinges), which will form convex polyhedra (with the panels becoming faces of the completed polyhedron) when folded along the fold lines and the edges of the polygonal boundary are joined together ${ }^{17}$. 


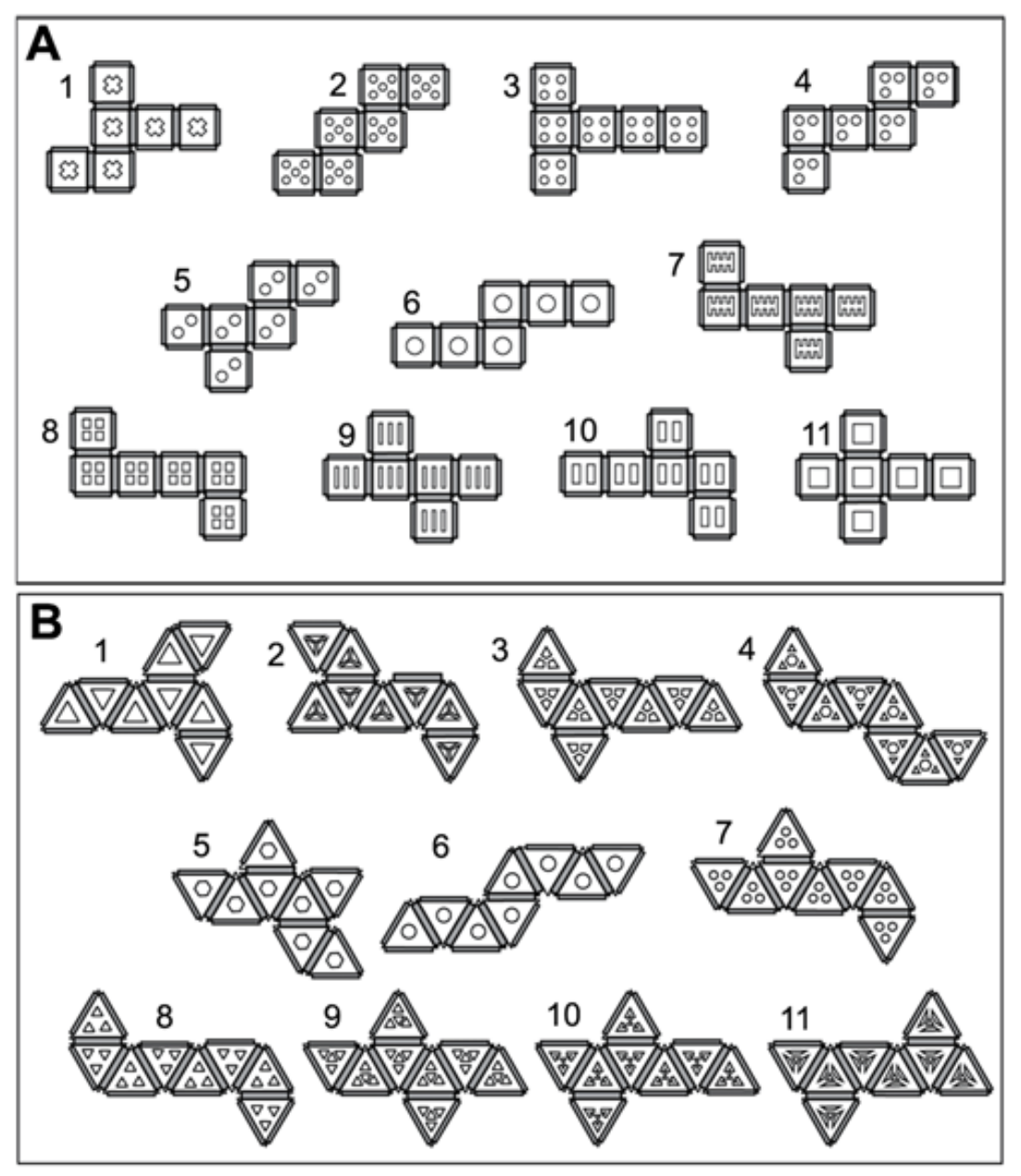

Figure T23. A) all (eleven) possible arrangements of 6 squares that can fold in a cube; $B)$ all (eleven) possible arrangements of 6 triangles that can fold in an octahedron $^{17}$

This situation is normally described by the term "net". The concept was proved on the example of self-folded cubes and octahedra. It involved Alexandrov's theorem-based model, predicting the possible arrangements that would lead to convex polyhedra after folding. The structures were then manufactured and their folding behavior was investigated in over 68 trials for each polyhedron. Special attention was paid to the number and type of defects observed in each trial. They found, that the radius of gyration had a major impact on the self-folding outcome, namely the smaller it was (i.e. the more compact the structure was), the greater the yield of properly assembled cubes and octahedra was. 


\section{Materials and Methods}

\section{Materials and}

\section{Methods}

\section{Results and}

\section{Discussion}

- Folding of rectangular films

- Folding of complex shapes. One-step folding

- Folding of complex shapes. Multi-step folding

- Multi-step folding of porous films

\section{Materials}

All substances used in this work are presented in the table below. The leftmost column gives the structural formula of the substance (where applicable), the second from left column includes the names of the chemicals; the third and the forth columns give the purity of the product when received and the deliverer, respectively. Some of the chemicals were purified prior to use; they are denoted with an * in the table. Information on the purification procedures is given in the text after the table. 
Table M1

List of materials used in the scope of this thesis

\begin{tabular}{|l|c|c|c|}
\hline & Name & Purity & Deliverer \\
\hline $\begin{array}{c}\text { Hydroxybenzop } \\
\text { henone }\end{array}$ & $98 \%$ & Aldrich \\
\hline $\begin{array}{c}\text { Acryloyl } \\
\text { chloride }\end{array}$ & p.a. & Sigma \\
\hline $\begin{array}{c}\text { N,N- } \\
\text { diisopropylethy } \\
\text { lamine }\end{array}$ & $99.5 \%$ & Sigma- \\
Aldrich
\end{tabular}


Table M1

List of materials used in the scope of this thesis

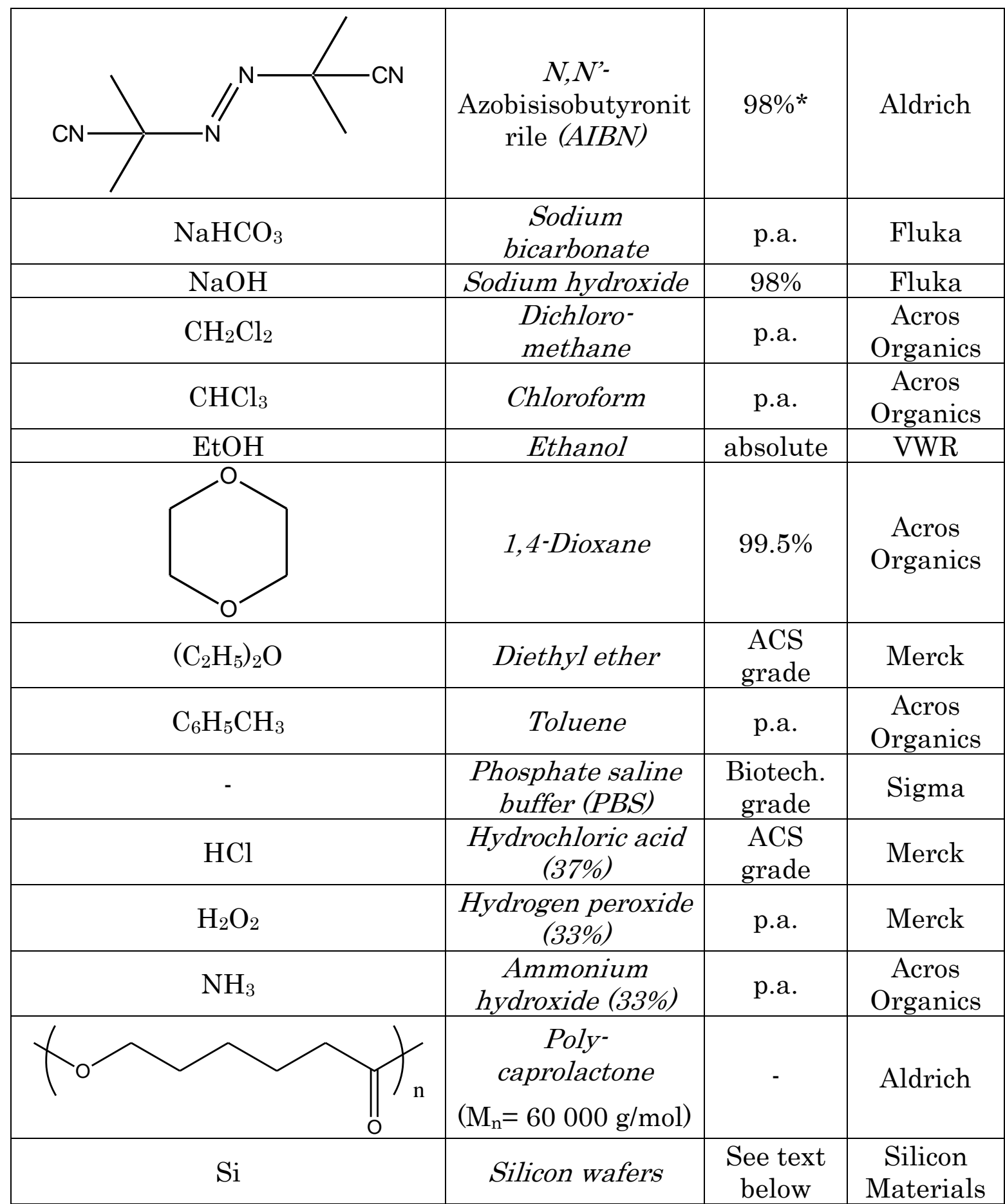

Millipore water: Obtained from TKA Smart2Pure; conductivity 0.055 $\mu \mathrm{S} / \mathrm{cm}$. 
Sodium hydroxide solution in water was used as a medium in some of the experiments. For this purpose, $20 \mathrm{mg}$ of sodium hydroxide were dissolved in $500 \mathrm{ml}$ Millipore water. The resultant solution had pH 11.

Cleaning of the silicon wafers: First, the silicon wafer was cut into pieces $4 \times 1.2 \mathrm{~cm}$. The pieces were then ultrasonicated in chlorophorm for 15 min. After this, they were immersed in a mixture of equal volumes of ammonium hydroxide, hydrogen peroxide and water and were stirred in it at $70^{\circ} \mathrm{C}$ for 1 hour. After cooling, they were rinsed twice with Millipore water and dried with compressed air. The cleanness of the surface was examined visually by the lack of stains and inhomogeneities of the reflecting layer of the wafer pieces. The thickness of the oxide layer on the surface of the wafers was measured by null-ellipsometry.

\section{Purification of the monomers}

Acrylic acid (AA): Acrylic acid was distilled under vacuum prior to use. In a typical procedure, $100 \mathrm{ml}$ of acrylic acid were poured into a $200 \mathrm{ml}$ bulb and connected to an oil-pump; the pressure was then reduced to $13 \mathrm{mbar}$. The acrylic acid was slowly heated to $40^{\circ} \mathrm{C}$. The first two milliliters of the distillate were thrown away. Distillation was stopped when approximately 10 $\mathrm{ml}$ of acrylic acid were left in the flask.

Methyl-mehtacrylate (MMA): Methyl-methacrylate was purified by passing it two times through a column with basic $\mathrm{Al}_{2} \mathrm{O}_{3}$ and one time through neutral $\mathrm{Al}_{2} \mathrm{O}_{3}$.

$N$-isopropylacrylamid $\quad(N I P A M): \quad \mathrm{N}$-isopropylacrylamide $\quad$ was recrystallized from hexane. In a typical experiment, a saturated solution of NIPAM in boiling hexane was prepared. The solution was then cooled down to room temperature and the NIPAM crystals were filtered out and dried under vacuum.

$N, N$ 'azobisisobutyronitrile (AIBN): AIBN was recrystallized from methanol. In a typical experiment, a saturated solution of AIBN in boiling methanol was prepared. The solution was then cooled down to room 
temperature and the AIBN crystals were filtered out and dried under vacuum.

Synthesis of 4-acryloylbenzophenone (BA): 4-Hydroxybenzophenone (20 g, $0.1009 \mathrm{~mol}), \mathrm{N}, \mathrm{N}$-diisopropylethylamine $(19.3 \mathrm{ml}, 0.1110 \mathrm{~mol})$ and $80 \mathrm{ml}$ of methylene chloride were added to a $200 \mathrm{ml}$ three-necked round-bottom flask fitted with an overhead stirrer, a thermometer, and an addition funnel with an acryloyl chloride $(9.02 \mathrm{ml}, 0.1110 \mathrm{~mol})$ solution in $20 \mathrm{ml}$ of methylene chloride. The acroloyl chloride solution was added dropwise into the flask under cooling $\left(0-5{ }^{\circ} \mathrm{C}\right)$ for ca $3 \mathrm{~h}$. The methylene chloride was removed by rotary evaporation. The residue was washed with $80 \mathrm{ml}$ of $20 \% \mathrm{HCl}, 80 \mathrm{ml}$ of saturated solution of sodium hydrocarbonate and dried over sodium sulfate. Next, the solution was passed through a silica gel column with chloroform as the eluent. Chloroform was removed by rotary evaporation. Finally, $24.44 \mathrm{~g}$ (95\%) of BA was obtained. 1H NMR (CDCl3, $500 \mathrm{MHz}): 6.05$ (dd, J1 1/4 10.40, J2 1/4 1.26, 1H), 6.34 (dd, J1 1/4 10.40, J3 1/4 17.34, 1H), 6.64 (dd, J3 1/4 17.34, J2 $1 / 41.26,1 \mathrm{H}), 7.27(\mathrm{~m}, 2 \mathrm{H}), 7.49(\mathrm{~m}, 2 \mathrm{H}), 7.59(\mathrm{~m}, 1 \mathrm{H}), 7.80(\mathrm{~m}, 2 \mathrm{H}), 7.86(\mathrm{~m}$, $2 \mathrm{H})$.

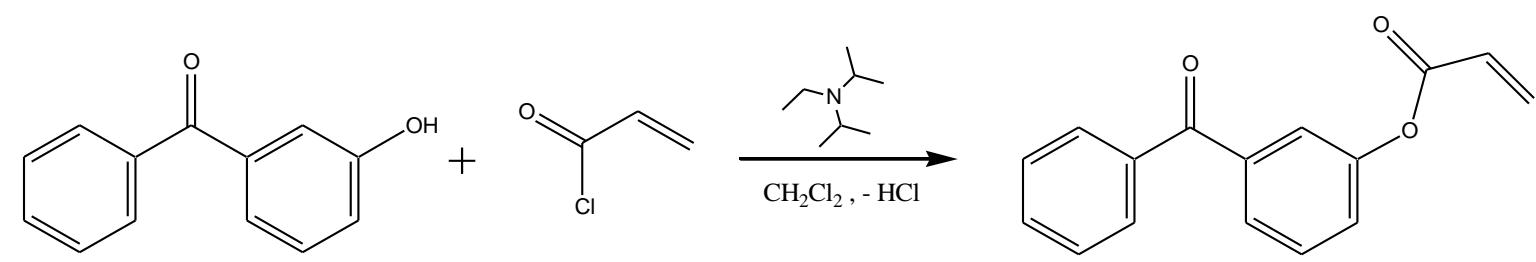

Figure E1. Synthesis of 4-benzophenone acrylate

\section{Preparation of polymer bilayers}

Two different techniques were used for the preparation of the polymer bilayers, namely dip-coating, either manual or with the use of a specially built for the purpose dip-coater, or by spin-coating. Spin-coating was used when films with a thickness of less than $100 \mathrm{~nm}$ had to be obtained. Manual dip-coating was used for the manufacturing of blends with thicknesses of 500 $\mathrm{nm}$ or more. In all other cases, the dip-coater was used. In all cases, precleaned silicon wafers were used as a substrate for the polymer bilayers. Any of the abovementioned techniques would be used to cast the first polymer on 
the smooth (mirror) side of the silicon wafer. The first layer always consisted of a PNIPAM-copolymer and was therefore coated from an ethanol solution. The second, passive, layer was either a PMMA-copolymer, or PCl with added 4-hydroxybenzophenone. A suitable solvent for the second polymer had to be found, as the first layer had to be kept intact during deposition. It was found by simple trial and error method that toluene was a good solvent for all the PMMA-copolymers, as well as for $\mathrm{PCl}$ and 4-hydroxybenzophenone, while being a non-solvent for PNIPAM and its copolymers, and was henceforth used as a medium for the solutions of the second polymer.

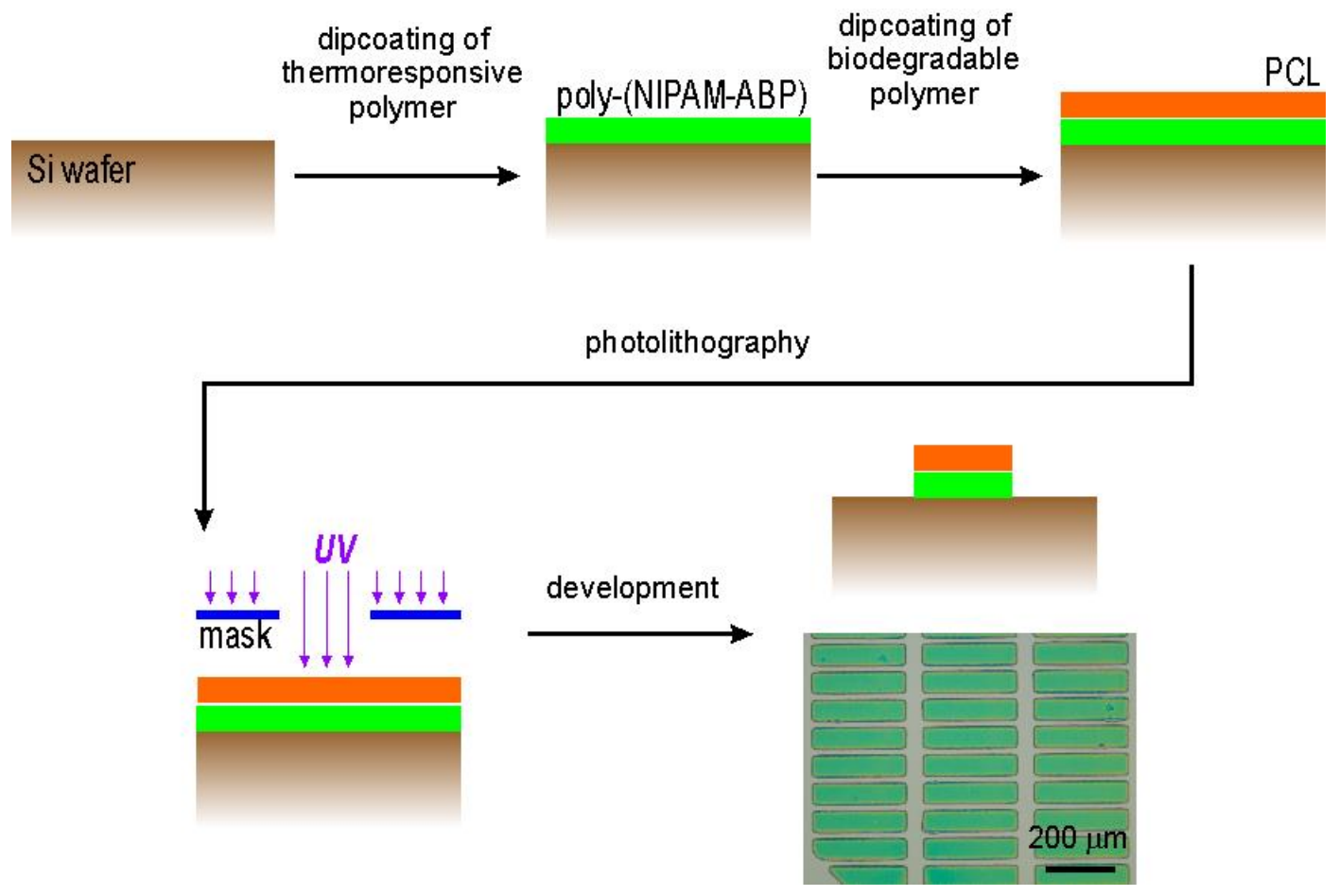

Figure E2. An illustration of the sample preparation. Two polymers are spinor dip-coated on the surface of a clean silicon wafer. The bilayer is then irradiated with UV light through a mask. The pattern is then developed in a common solvent.

All solutions were filtered through a $200 \mu \mathrm{m}$ PTFE filter prior to use. The bilayer film was then illuminated with $254 \mathrm{~nm}$ UV-light through specially designed photomasks for $90 \mathrm{~min}$ to cross-link the polymers. 4hydroxybenzophenone, as well as its acrylate, form radicals under UV-light: 
<smiles>[R]Oc1cccc(C(=O)c2cccc(C(=O)c3ccccc3)c2)c1</smiles>

Figure E3. Radical formation for benzophenone derivatives under irradiation with UV-light

Afterwards, the illuminated film was rinsed with dichloromethane in order to remove the polymers from the non-irradiated areas.

\section{Characterization Techniques}

\section{Null-ellipsometry}

Layer thickness of thin (less than $400 \mathrm{~nm}$ ) films was measured by nullellipsometry, which is a non-destructive technique for the characterization of thin polymer layers on solid substrates.

\section{Basics}

Incident polarized laser light is reflected off a sample, usually compensated by an optical compensator. The reflected beam forms an ellipse vertically with respect to the light propagation whose parameters (length of the ellipse axes and rotation angle of the ellipse with respect to vertical surface) can be detected by a rotating polarizer, the so called analyzer.

The position of polarizer and analyzer at minimum and maximum intensity give the phase parameter and the amplitude parameter that depend on the sample characteristics which are the layer thickness and the refractive index by measuring the change of phase between the s (perpendicular) and the $\mathrm{p}$ (parallel) components of light (Figure E4). All measurements were performed at $632.8 \mathrm{~nm}$ and $70^{\circ}$ angle of incident light with a null ellipsometer in a polarizer compensator-sample analyzer (Multiscope, Optrel Berlin) as described elsewhere ${ }^{158}$. 


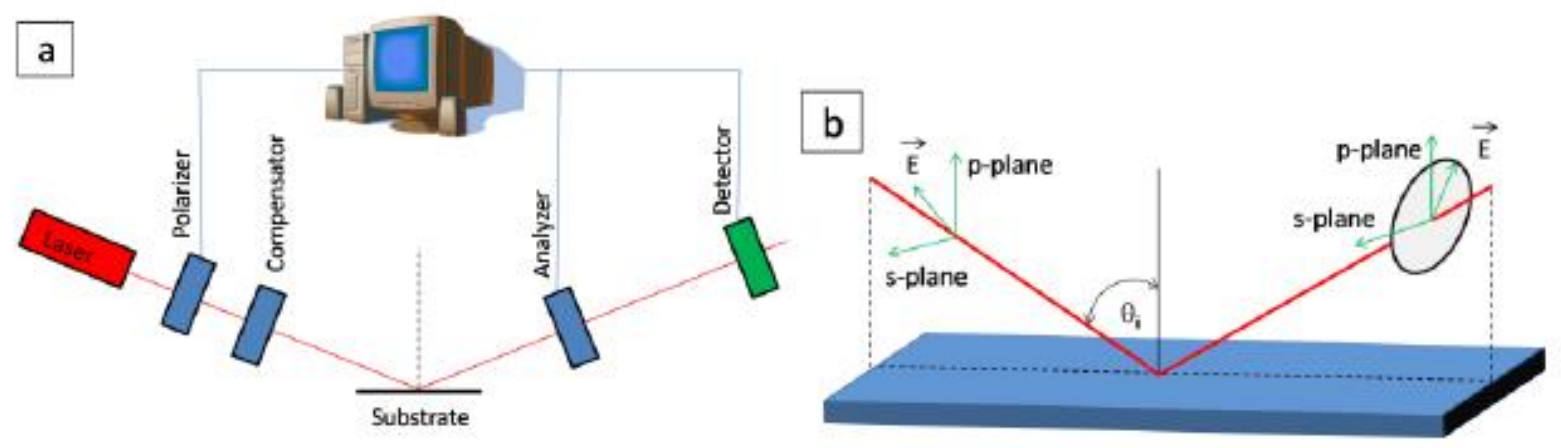

Figure E4: Principle of null-ellipsometry (simplified) (a), schematic of an ellipsometry experiments (b).

\section{Scanning electron microscopy}

\section{Basics}

The principle of a scanning electron microscope is shown schematically in Figure E4. The two major parts are the microscope column and the electronics console. The microscope column consists of the electron gun (with the components cathode, Wehnelt cylinder, anode), one or two condenser lenses, two pairs of beam deflection coils (scan coils for $X, Y$ deflection), the objective lens, and some apertures. In the specimen chamber at the lower end of the microscope column are located the specimen stage and the detectors for the different signals generated by the electron-specimen interaction. The pressure in the specimen chamber typically amounts to about $10^{-6} \mathrm{~Pa}$, allowing the beam electrons to travel from the cathode to the specimen with little interaction with the residual gas molecules. The electronics console consists of the electric power supplies for the acceleration voltage (usual range about $0.5-30 \mathrm{kV}$ ) as well as the condenser and objective lenses, the scan generator, and electronic amplifiers for the different signals acquired. 


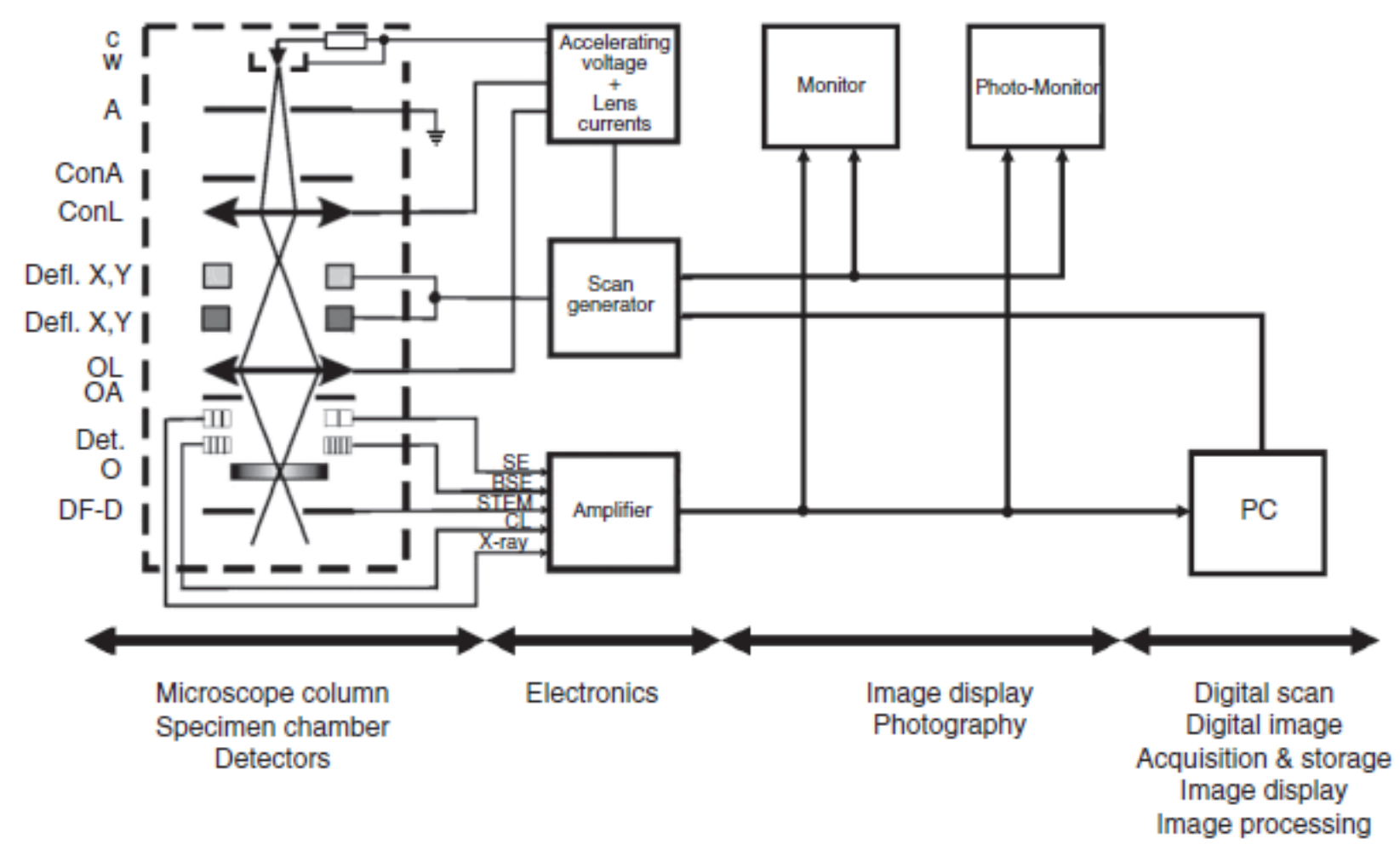

Figure E5. Diagram of a typical SEM ${ }^{159}$

Modern SEMs mostly use a PC to control the electron beam, to select the signals, and to record as well as to store the digital image(s). How does the SEM work? The beam electrons are emitted from the cathode and accelerated by a voltage of $0.5-30 \mathrm{kV}$ between the cathode and anode forming a smallest beam cross-section-the crossover-near the anode with a diameter of about $10-50 \mu \mathrm{m}$. This spot size is too large to produce a sharp image. Therefore the crossover is demagnified by the lens system consisting of one or two condenser lenses and one objective lens and focused on the specimen surface. Topography is the factor that contributes most amongst the others to the image contrast. The magnification of the image is given by the ratio of the length of the scan on the monitor and the corresponding length of the scan on the specimen. For example, an increase in magnification can simply be achieved by decreasing the current of the deflection coils in the microscope column (i.e., lowering the length of the scan on the specimen) and keeping the image size on the monitor constant. 
One of the greatest strengths of the SEM is the tremendous depth of focus, i.e., the range of heights of the specimen being simultaneously in focus. At magnifications that are comparable to those of light microscopy (e.g., 100x) the SEM has a depth of focus that is about 100 times greater than that of an optical microscope ${ }^{159}$.

Null-ellipsometry is not suitable for measuring the thickness of polymer layers which are thicker than $500 \mathrm{~nm}$ as several artifacts may occur. To overcome these problems, we decided to use SEM to directly measure the thickness of the polymer films. For this purpose, the substrate with the polymer bilayer was first cooled with liquid nitrogen and then quickly broken into pieces. Different parts were then picked up and viewed perpendicular to the film plane. Cooling with liquid nitrogen was essential for specimens, where the second layer was $\mathrm{PCl}$, because of the polymer's low glass-transition temperature. If broken at room temperature, the PCl layer stretched and often even detached from the lower layer.

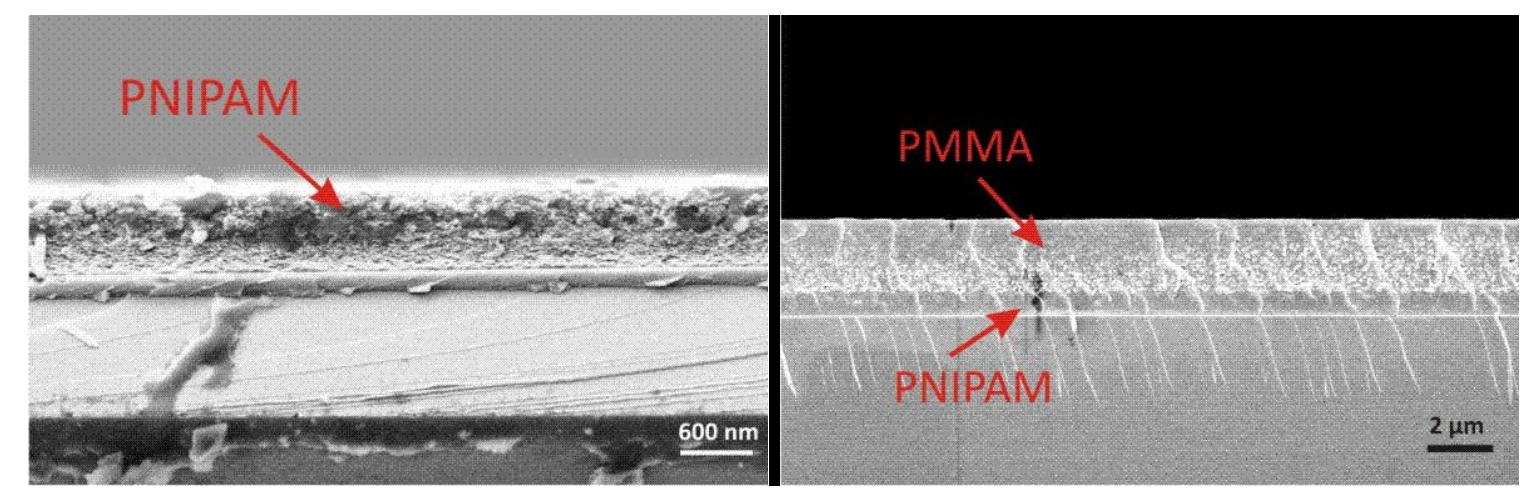

Figure E6. SEM images of $P(N I P A M-A A-B A)$ (left) and P(NIPAM-AA-BA)$P(M M A-B A)$ (right) on a silicon wafer

Figure E6 shows typical SEM images of a polymer film on a silicon wafer. The polymer layer (the topmost layer on the left image) can be clearly distinguished from the substrate. Moreover, it was possible to easily distinguish between the two polymers, as seen on the right image.

All scanning electron microscopy (SEM) micrographs were acquired on a Neon40 SEM (Zeiss), operating at $3 \mathrm{kV}$ in the secondary electron and InLens modes. All samples were covered with $3.5 \mathrm{~nm}$ of $\mathrm{Pt}$ prior to imaging. 


\section{Visible light microscopy}

\section{Basics}

Optical microscopes are instruments which uses visible light and a system of lenses to magnify images of small samples. The main factors that determine the quality of an image are the observed object itself, with its contrast in respect to its internal or external surroundings, as well as the magnification and the resolving power of the microscope. A typical set-up of a modern microscope includes a light source (usually with collector and condenser lenses, required for Köhler illumination), an objective lens, an eyepiece, a detector (either observer's eye or a camera) and a stage for the specimen.

\section{Light source}

There are plenty of light sources used in light microscopy like ambient light, halogen lamps, mercury lamps, to name a few. A common problem of all light sources is that an image of the filament is visible in the image of the sample. To overcome this problem, the so called Köhler illumination is used in modern microscopes. Köhler illumination requires several optical components to function:

1. Collector lens and/or field lens

2. Field diaphragm

3. Condenser diaphragm

4. Condenser lens

These components lie in this order between the light source and the specimen and control the illumination of the specimen. The collector/field lenses act to collect light from the light source and focus it at the plane of the condenser diaphragm. The condenser lens acts to project this light, without focusing it, through the sample. This illumination scheme creates two sets of conjugate image planes, one with the light source image and one with the specimen. The primary advantage of Köhler illumination is the extremely even illumination of the sample. This reduces image artifacts and provides high sample contrast. 


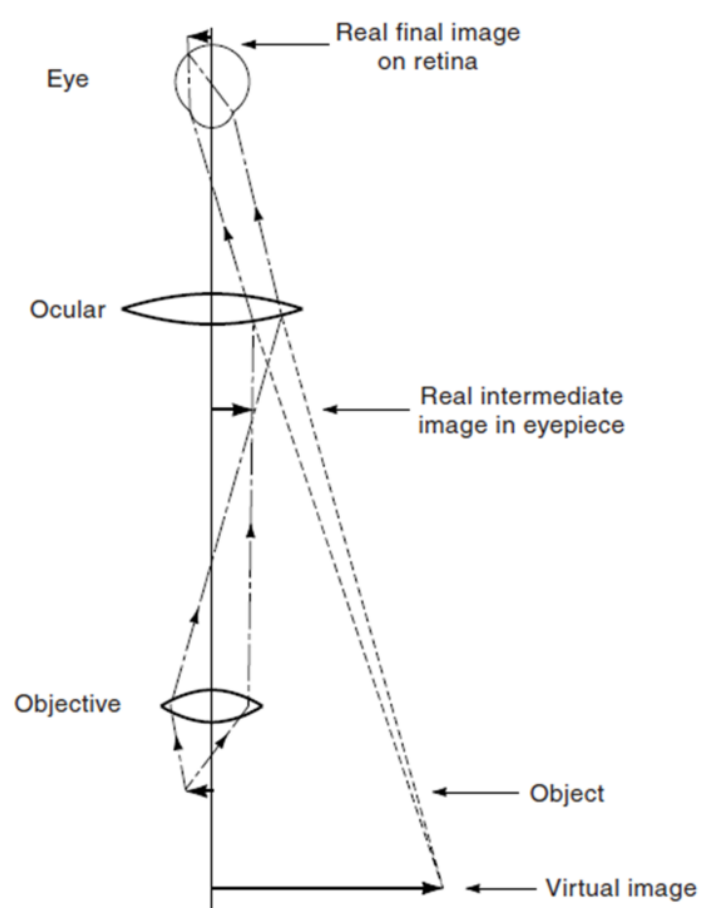

Figure E7. Basic elements of a typical visible light microscope
Eyepiece (ocular)

The eyepiece, or ocular, is a cylinder containing two or more lenses. Its function is to bring the image into focus for the eye. Eyepieces are usually interchangeable and many different eyepieces can be inserted with different degrees of magnification. Typical magnification values for eyepieces range between $2 \mathrm{x}$ and $10 \mathrm{x}$.

\section{Objective lens}

The objective, which is typically composed of several lens elements, collects light transmitted through or reflected

from the specimen and forms a magnified real image at the real intermediate image plane near the eyepieces. The intermediate image is then further magnified by the eyepiece to produce a secondary enlarged virtual image of the specimen, which is observed by the microscopist.

The objective lens forms a magnified image of the object (called the real intermediate image) in or near the eyepiece; the intermediate image is examined by the eyepiece and eye, which together form a real image on the retina. Because of the perspective, the retina and brain interpret the scene as a magnified virtual image about $25 \mathrm{~cm}$ in front of eyes.

\section{Magnification}

The actual magnification of a compound optical microscope is the product of the powers of the ocular and the objective lens. The maximum normal magnifications of the ocular and objective are 10x and 100x respectively, giving a final magnification of $1,000 x$. If a detector is used, the 
image is not magnified by the eyepiece, so a typical magnification in this case would be 100x.

\section{Resolution}

Resolution, or minimum resolving distance, is the minimum distance between two points in an image, at which they can still be seen as separate objects. At high magnifications, point objects are seen as fuzzy discs surrounded by diffraction rings, called Airy discs. The resolving power of a microscope is taken as the ability to distinguish between two closely spaced Airy disks. In other words, it is diffraction that limits the ability to resolve fine details. The extent and magnitude of the diffraction patterns are affected by both the wavelength of light $(\lambda)$ and the numerical aperture (NA) of the objective lens. There is therefore a finite limit beyond which it is impossible to resolve separate points in the objective field, known as the diffraction limit. Assuming that optical aberrations in the whole optical set-up are negligible, the resolution $\mathrm{d}$, can be stated as:

$$
d=\frac{\lambda}{2 N A}
$$

Usually a wavelength of $550 \mathrm{~nm}$ is assumed, which corresponds to green light. With air as the external medium, the highest practical NA is 0.95, and with oil, up to 1.5. In practice the lowest value of $\mathrm{d}$ obtainable with conventional lenses is about $200 \mathrm{~nm}$.

\section{Bright-field}

In bright-field microscopy, contrast results from absorbance of illuminating light in dense areas of the specimen. The background is bright, and absorbing structures within the specimen appear darker. Thus, brightfield microscopy is ideal for objects with high natural absorption. 


\section{Dark field}

To introduce contrast into transparent colorless samples dark-field imaging can be used. In dark-field microscopy the image is formed by collecting only the scattered light and excluding the transmitted. As a result, only highly diffracting or scattering structures can be observed forming a bright image on a dark background. Dark field illumination technique is especially good to reveal outlines, edges, and boundaries of objects.

For light microscopy imaging a Zeiss Axio microscope equipped with Zeiss 5x, 10x, 20x, 50x and 100x air objectives was used.

To perform microscopic observation at different temperatures samples were placed on a home-made thermo-stage based on a Peltier element, which allows a very fast change of the temperature within several seconds.

LM images were taken with an Olympus UC-30 3.2 megapixel digital color camera with CCD chip. AnalySIS software (Olympus Soft Imaging Solutions, V5.1) and ImageJ freeware were used for data processing. 


\section{Results and Discussion}

\section{Folding of Rectangular Bilayers}

\section{Motivation}

Generally, the rolling of a rectangular bilayer may occur according to three different scenarios: long-side, short- side, and diagonal rolling (see Figure R1). The effects of film shape on the character of folding were experimentally investigated on the examples of purely inorganic and composite polyaniline -inorganic bilayers. Smela et al. ${ }^{68}$ showed that shortside rolling was preferred in the case of free homogeneous actuation and that this preference increased with the aspect ratio (ratio of length to width of a rectangular pattern). On the other hand, $\mathrm{Li}$ et al. experimentally demonstrated the opposite scenario 69 in the case where bilayers are progressively etched from a substrate, namely, a preference for long-side rolling. They observed that when the tube circumference was much larger than the width, or the aspect ratio of the rectangle was high, rolling always occurred from the long side. However, when the tube circumference was much smaller than the width and the aspect ratio of the rectangular bilayer was not very high, the rolling resulted in a mixed yield both of long and short-side rolling, as well as a "dead-locked turnover" shape. Elseways, short-side rolling occurred at small aspect ratios when the deformed circumference was close to the width. In these self-rolling systems, the active component undergoes relatively small volume changes or actuation strains, which are nearly homogeneous over the whole sample. 


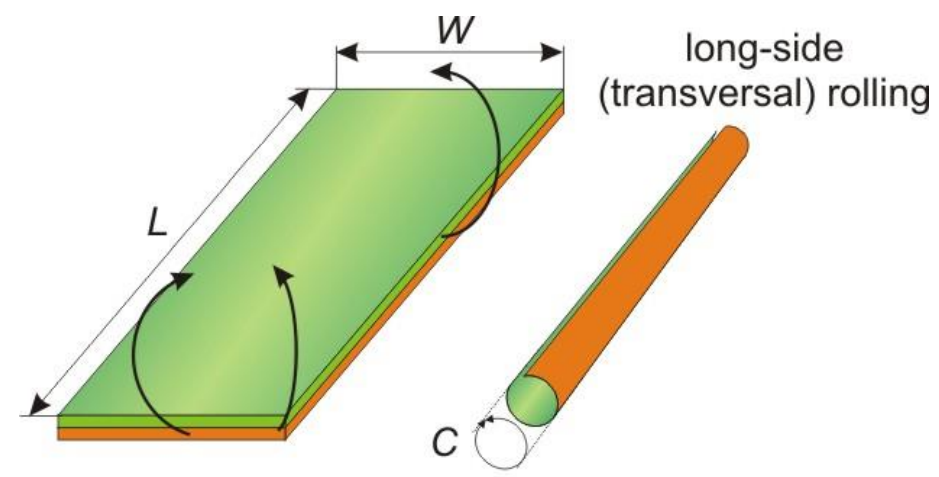

short-side diagonal rolling (longitudinal) rolling
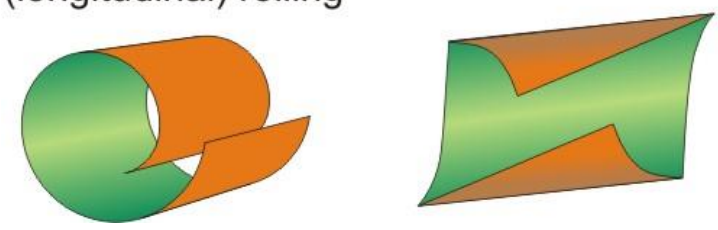

Figure R1. Different scenarios for folding of polymer bilayers

Hydrogel films, which are also able to fold, demonstrate considerably different properties ${ }^{132,139,160}$. First, hydrogels undergo large volume changes (up to 10 times) upon swelling and contraction. Second, the swelling of a hydrogel is often kinetically limited: due to slow diffusion of water through a hydrogel, the parts that are closer to the edges swell first, while the parts that are closer to the center of the film swell later. Thus, the actuation profile inside the active layer is heterogeneous. A conclusion may be drawn that, before designing a complex self-folding system based on a polymer bilayer, the phenomena that underlie and determine the process should be thoroughly investigated and understood. To attack the problem, one of the simplest geometrical forms was chosen - a rectangle.

Experimental part

Synthesis of P(MMA-BA): $6.2803 \mathrm{~g}$ of MMA (62.72 mmol), $0.2405 \mathrm{~g}$ of BA $(0.96 \mathrm{mmol})$, and $0.052 \mathrm{~g}$ of AIBN $(0.31 \mathrm{mmol})$ were dissolved in $30 \mathrm{~mL}$ of toluene. The mixture was purged with nitrogen for $30 \mathrm{~min}$. The polymerization was carried out at $70^{\circ} \mathrm{C}$ under a nitrogen atmosphere with mechanical stirring overnight. After cooling, the mixture was poured in 750 
$\mathrm{mL}$ of diethyl ether, and the precipitate was filtered and dried under vacuum at $40^{\circ} \mathrm{C}$.

Synthesis of P(NIPAM-AA-BA) : BA (0.28 g, 1,12 mmol); NIPAM (6 g, $51.57 \mathrm{mmol})$, AA (0.2556 g, $3.36 \mathrm{mmol})$, AIBN (0.01632 g, $0.38 \mathrm{mmmol})$ were added in a $50 \mathrm{~mL}$ flask. The components were dissolved in $30 \mathrm{~mL}$ ethanol. The mixture was purged with nitrogen for $30 \mathrm{~min}$. The polymerization was carried out at $70^{\circ} \mathrm{C}$ under nitrogen atmosphere with mechanical stirring overnight. After cooling, the mixture was poured in $750 \mathrm{~mL}$ diethyl ether, the precipitate was filtered and dried in vacuum at $40^{\circ} \mathrm{C}$.

\section{Results and discussion}

As no data on the dependence of the folding diameter on the film thicknesses was available prior to the experiment, series of solutions of $\mathrm{P}$ (NIPAM-BA) in ethanol and $\mathrm{P}(\mathrm{MMA}-\mathrm{BA})$ in toluene were prepared. Table E1 gives a summary of the concentrations of the prepared polymer solutions and the resulting thicknesses of the polymer films, formed after dip-coating in the corresponding solution.

Table RT1

Dependence of the thickness of the polymer films on the concentration of the stock solution

\begin{tabular}{|c|c|c|c|c|c|}
\hline & Conc., \% & 2 & 4 & 6 & 8 \\
\hline $\begin{array}{c}\text { P(NIPAM- } \\
\text { AA-BA })\end{array}$ & Thickness, nm & $215 \pm 15$ & $600 \pm 50$ & $1200 \pm 200$ & $2500 \pm 200$ \\
\hline P(MMA-BA) & Thickness, nm & $200 \pm 20$ & $450 \pm 30$ & $680 \pm 40$ & $1230 \pm 80$ \\
\hline
\end{tabular}

As manual dip-coating results in a thickness-gradient of the polymer film, different values were measured in the top, middle and bottom parts of the silicon piece. The values presented in the table are the thicknesses of the polymer film in the middle of the sample and the " \pm " sign represents the increase or decrease in the thickness towards the bottom and top part of the wafer, respectively. 
Combining these solutions, thirteen different polymer bilayers were prepared to test their folding ability in PBS buffer ( $\mathrm{pH}$ 7.4) and to determine the diameter of folding. Table RT2 gives a summary of the folding behavior and diameters (in $\mu \mathrm{m}$ ) for the cases where rolling was observed:

Table RT2

Radii of folding of different polymer bilayer

\begin{tabular}{|c|c|c|c|c|}
\hline $\begin{array}{c}\mathrm{w}(\text { PNIPAM), } \\
w(\text { PMMA), } \%\end{array}$ & $2 \%$ & $4 \%$ & $6 \%$ & $8 \%$ \\
\hline $2 \%$ & No folding & $25-40 \mu \mathrm{m}$ & $7-10 \mu \mathrm{m}$ & $<5 \mu \mathrm{m}$ \\
\hline $4 \%$ & No folding & $35-50 \mu \mathrm{m}$ & $18-22 \mu \mathrm{m}$ & $5-10 \mu \mathrm{m}$ \\
\hline $6 \%$ & $-*$ & No folding & $40-80 \mu \mathrm{m}$ & $x^{* *}$ \\
\hline $8 \%$ & - & - & $70-90 \mu \mathrm{m}$ & $x$ \\
\hline
\end{tabular}

*Dashes denote that no experiments with that combination of concentrations were conducted **An " $x$ " connotes that the structures detached during development

We chose two of the systems, namely bilayers $6-4$ and $6-8$, because they gave the lowest deviation in terms of radius of folding. Accordingly, we prepared two sets of patterned bilayers of P(NIPAM-AA-BA)/(P(MMA-BA), which differ in thickness (h) of the $\mathrm{P}(\mathrm{MMA}-\mathrm{BA})$ layer, which results in different rolling curvature. One set formed narrow tubes with a diameter $\mathrm{D}=$ $20 \mu \mathrm{m}\left(\mathrm{h}_{\mathrm{P}(\mathrm{MMA}-\mathrm{BA})}=450 \mathrm{~nm} ; \mathrm{h}_{\mathrm{P}(\mathrm{NIPAM}-\mathrm{AA}-\mathrm{BA})}=1200 \mathrm{~nm}\right)$, while the second set formed wider tubes with diameters in the range $d=70-90 \mu \mathrm{m}\left(\mathrm{h}_{\mathrm{P}}(\mathrm{MMA}-\mathrm{BA})=\right.$ $1200 \mathrm{~nm} ; \mathrm{hP}($ NIPAM-AA-BA $)=1200 \mathrm{~nm})$. Additionally, thermoresponsive hydrogels swell and shrink at reduced and elevated temperature, respectively. The passive hydrophobic $\mathrm{P}(\mathrm{MMA}-\mathrm{BA})$ layer restricts swelling of the active hydrogel. As a result, the bilayer made of these polymers does not uniformly expand/shrink but folds and unfolds due to the swelling and collapse of the hydrogel layer. P(NIPAM-AA-BA)/(P(MMA-BA) bilayers were prepared using photolithography, as described in the experimental part. Rectangular bilayers of different lengths $(\mathrm{L}=100-1000 \mu \mathrm{m})$ and aspect ratios (ratio of length (L) to width $(\mathrm{W}), \mathrm{A}=\mathrm{L} / \mathrm{W}=1-8$ ) were fabricated using 
specially designed photomasks. After removal of the non-crosslinked polymer, the patterned bilayers were exposed to a PBS solution $(\mathrm{pH}=7.4)$ at room temperature. As a result, photo-crosslinked P(NIPAM-AA-BA) swelled, leading to rolling of the bilayer and formation of tubes. The folded films formed by each set of bilayers were then mapped by optical microscopy in order to assess the rolling radius as well as the deformation pattern (see Figures R2 and R3).

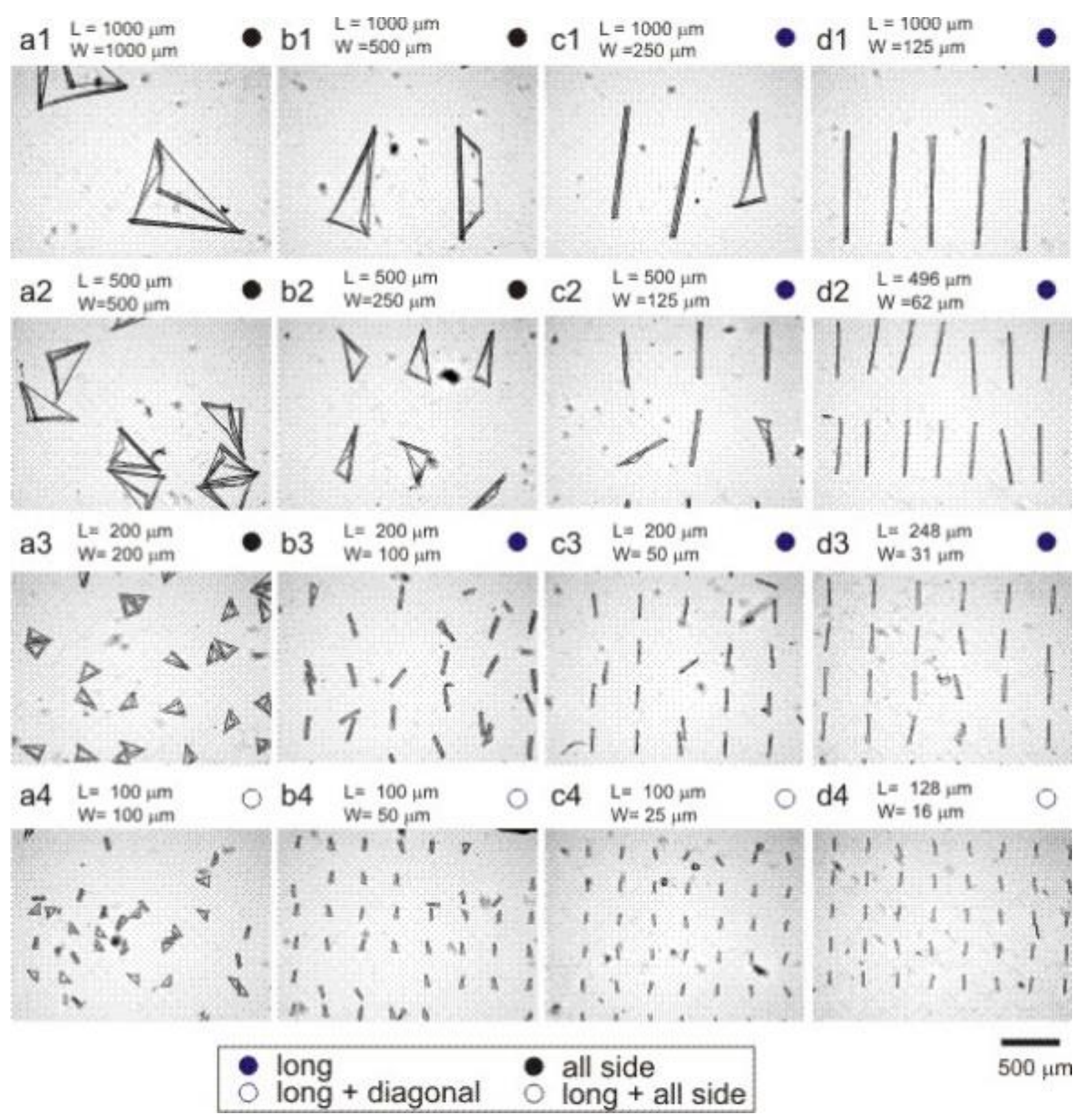

Figure R2. Folding behavior of rectangular bilayers with radius of folding 18$22 \mu \mathrm{m}$. 


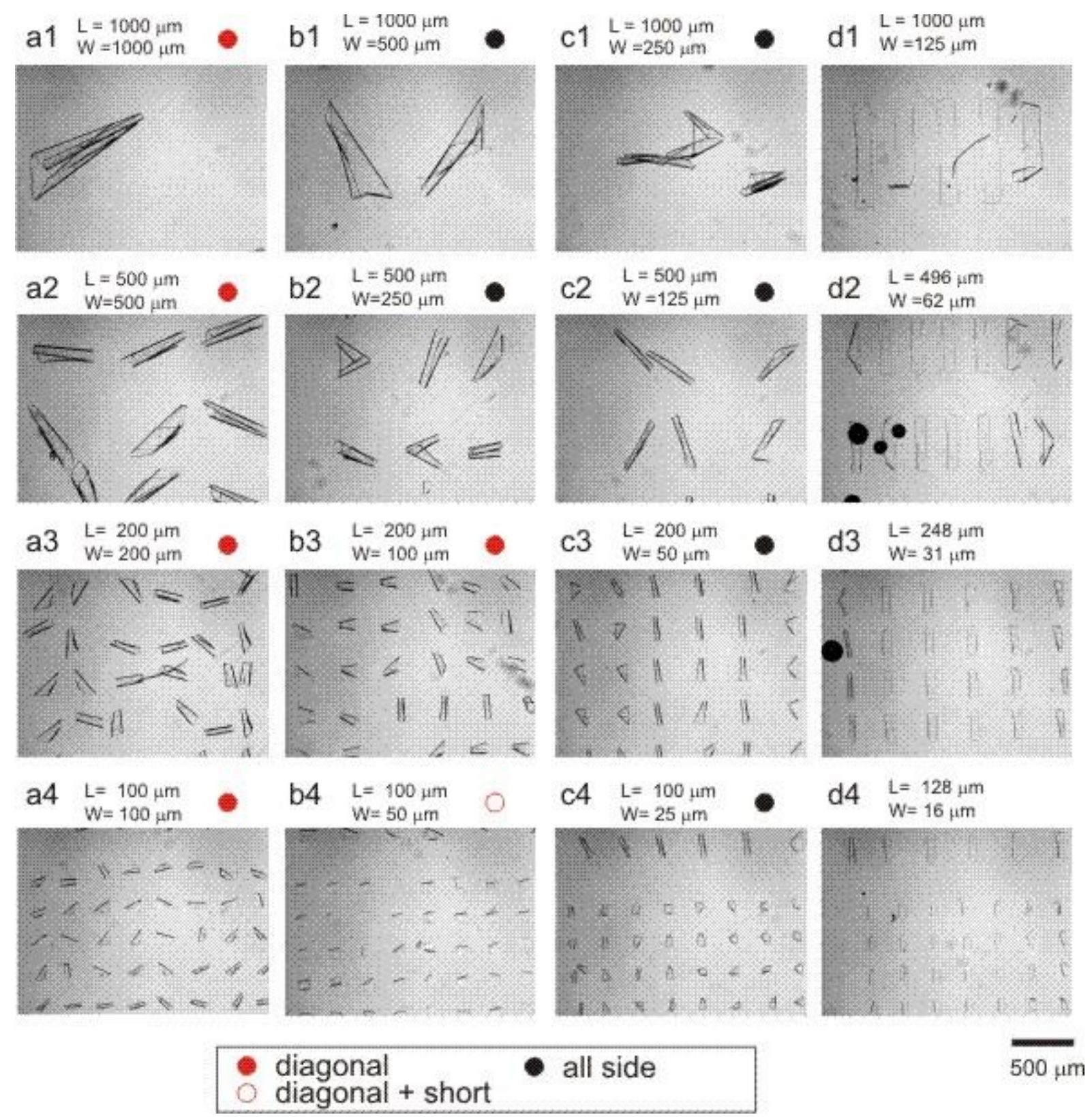

Figure R3. Folding behavior of rectangular bilayers with radius of folding 70$90 \mu \mathrm{m}$.

It was found that the final diameter of the tube is independent from the size of the bilayer (L, W) and, when everything else being equal (Young modulus of active and passive layer as well as activation strain), it is solely controlled by the relative thickness of the active and passive layers and, thus, is (almost) constant for each set of experiments. On the other hand, the direction of rolling strongly depends on the size and shape of the films as well as on the thicknesses of the active and passive layer (sees Figure R4). 


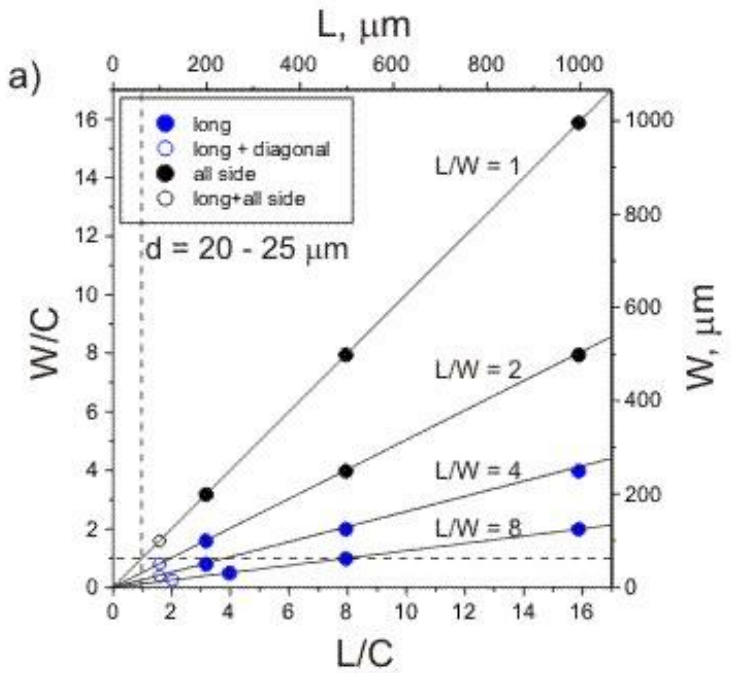

b)
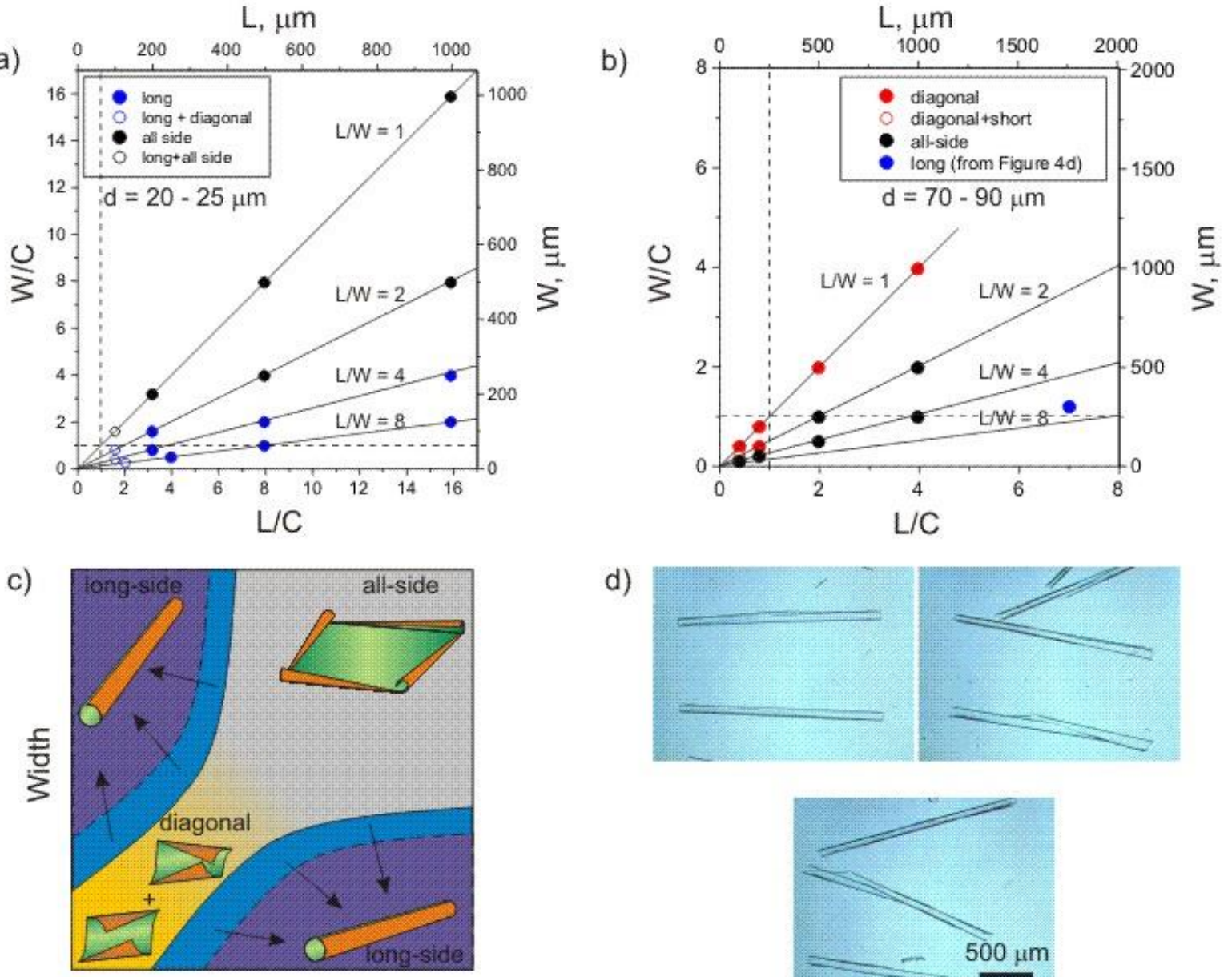

d)

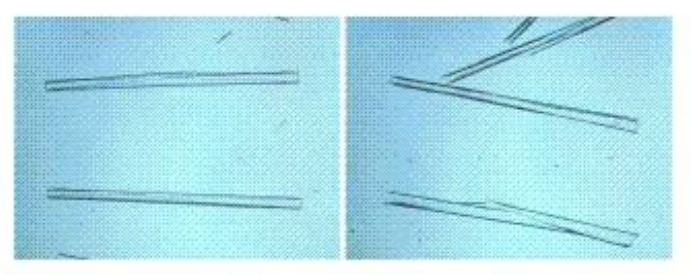

Length

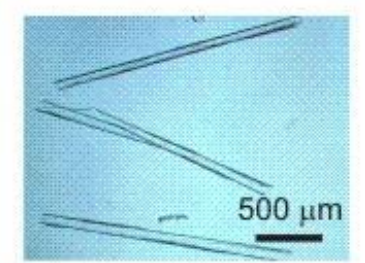

Figure R4. Phase diagrams of the folding behavior of rectangular polymer bilayers with different circumferences. a) diameter of folding 20-25 $\mu \mathrm{m}$; b) diameter of folding $70-90 \mu \mathrm{m}$; c) summarized phase diagram; d) an example of rolling of rectangular polymer bilayers with aspect ratio 1/8.

Summarizing, we distinguished four general types of rolling: long-side rolling, diagonal rolling, short-side rolling, and mixed all-side rolling, which is a combination of the first three types. The character of preferential rolling is plotted as a function of the absolute values of width, length and aspect ratio, as well as normalized values, which are obtained by dividing the length or width by the typical circumference of the rolled tube $(C=\Pi D$, Figure $\mathrm{R} 1)$. Three types of rolling were observed when narrow tubes $(\mathrm{D}=20 \mu \mathrm{m})$ are formed: long-side, diagonal, and all-side rolling (see Figure R4a). It must be noted that no short-side rolling was observed. The all-side rolling (see Figure R2, a1-3, b1-2) occurs when the width of the films considerably exceeds the circumference of rolling for aspect ratios of 1 or 2 . A decrease in the width for 
an aspect ratio of 2 or more results in preferential long-side rolling (see Figure R2, b3, c1-3, d1-3), when the normalized length is more than 2 . Depending on the ratio of width (W) to circumference $(\mathrm{C})$, incompletely rolled tubes $(\mathrm{W} / \mathrm{C}<1)$, completely rolled tubes $(\mathrm{W} / \mathrm{C} \approx 1)$, or doubled tubes $(\mathrm{W} / \mathrm{C}=2)$ are formed. A further decrease in the length leads to a mixture of long-side and diagonal or all-side rolling (see Figure 2, a4, b4, c4, and d4). Thus, the most promising parametric window for potential applications, such as microfluidics and cell encapsulation, is a bilayer with an aspect ratio of 4 or more. Different rolling behavior is observed when wide tubes $(\mathrm{D}=70-90 \mu \mathrm{m})$ are formed (see Figure R4b). First, films with the highest aspect ratio slightly bend and almost do not roll because of the large circumference (see Figure R2 (b), d1-4). Second, other bilayers roll either according to diagonal or all-side rolling scenarios. Diagonal rolling is observed in the cases of square films $(\mathrm{L} / \mathrm{W}=1)$ when two opposite corners bend toward each other (see Figure R2 (b), a1-4). Another interesting rolling mechanism was the double diagonal rolling, where all four vertexes of the rectangle bend towards the center of the figure.

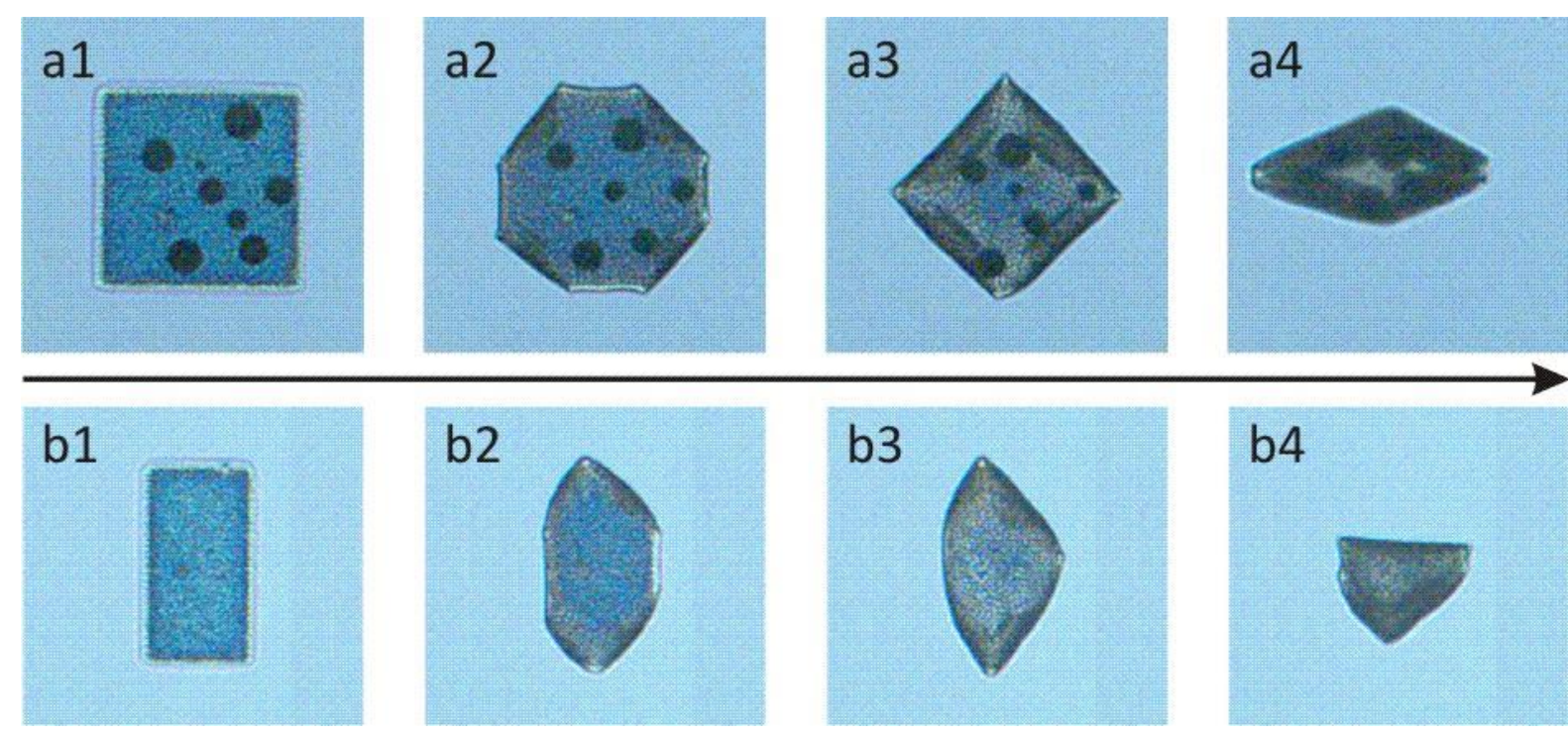

Figure R5. Examples of double diagonal rolling for rectangular polymer bilayers with aspect ratios 1/1 (a1-a4) and 1/2 (b1-b4)

Overall, such type of rolling also occurs at low aspect ratios (1 or 2) when the radius of folding is comparable to the length of the structures. Here, 
folding starts from the corners and proceeds until the rolling of tubes. As a result, a square or a rectangle is formed, just like in the case of all-side rolling. The difference between the two scenarios is that in all-side rolling, the sides of the rectangles are formed by rolling of the sides of the initial structure, while in the double diagonal folding they are formed from the corners.

"Tick-or-check-mark"-like structures (see for example Figure R2b, c1, the film in the middle) in combination with diagonal rolling are observed in almost all cases at $\mathrm{L} / \mathrm{W}>1$ when either adjacent or opposite corners bend toward each other. Bending from short sides was observed in combination with diagonal rolling only in one case (see Figure R2b, b4). The results obtained for narrow (Figure R2a and R4a) and wide (Figure R2b and R4b) tubes plotted as a function of normalized length and width are not fully identical. Figure R4b is shifted to larger values of $\mathrm{L} / \mathrm{C}$. The reason for this effect is not completely clear and could be due to effects related to heterogeneities in the swelling behavior, which are hard to fully consider. On the other hand, there is a clear correlation between the results given in Figure R4a and b, which is qualitatively summed up in Figure R4c. For example, all-side rolling is observed when both length and width considerably exceed the deformed circumference. Diagonal rolling is observed when $\mathrm{L}=\mathrm{W}$, and both are comparable to the circumference. Mixtures of diagonal rolling and the formation of "tick-or-check-mark"-like structures (tube in the middle of Figure 3, c1) are observed when $\mathrm{L}>\mathrm{W}$ and both $\mathrm{L}$ and $\mathrm{W}$ are comparable to the circumference.

In order to explain the fact that rolling starts from the corners we experimentally investigated the swelling process. This was performed in a qualitative manner by observing changes in the interference pattern of white light with the bilayer during swelling. In order to avoid bending and folding of the bilayer during swelling, a very thin P(NIPAM-AA-BA) layer $(\mathrm{h}=35$ $\mathrm{nm})$ under a thick P(MMA-BA) layer $(\mathrm{h}=400 \mathrm{~nm})$ was used. As one can see from Table RT2, a combination of these thicknesses leads to a non-folding polymer bilayer. Due to the effect of interference of light, which is mirrored 
from the top and bottom surfaces of the bilayer, the changes in colors (see Figure R6) reflect the changes in the film thickness. It was observed that the color of the films started to change at the corners first, which confirmed the assumption that edge-activation of the active layer due to slow water diffusion into the hydrogels is at the origin of the experimentally observed fact that rolling starts at the corners.

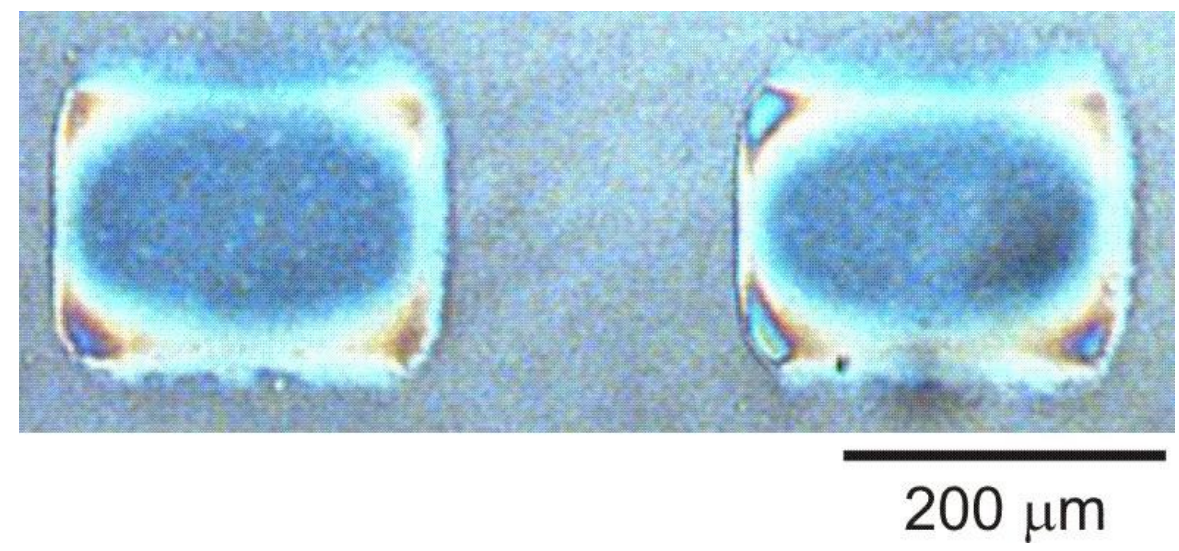

Figure R6. Swelling of a rectangular polymer bilayer. At the beginning, a change in colour is observed in the corners.

Thus, on the basis of the observations of the rolling and swelling mechanisms, we can argue that diffusion determines folding in the first moments of folding, while adhesion seems to play a decisive role at later stages of folding.

Diffusion-Driven Actuation: For some specific shape parameters the observed long-side folding of rectangular bilayers contradicts with the reported in literature bending of bilayer actuators, which occurs along the short side. However, the latter is true under the conditions that the active layer is homogeneously activated and that there is no interaction with a substrate. 
a) homogenous swelling of freely floating bilayer

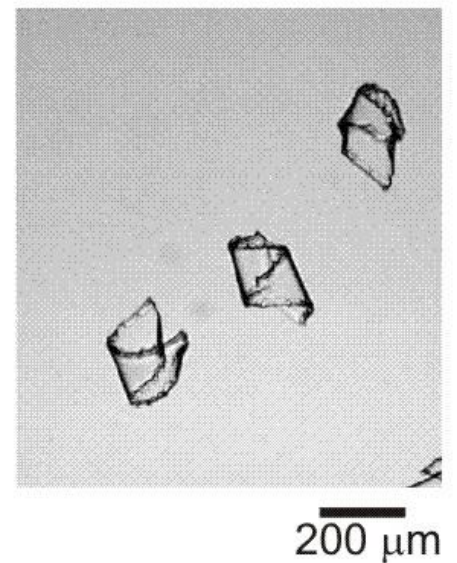

b) inhomogenous swelling of bilayer on the substrate

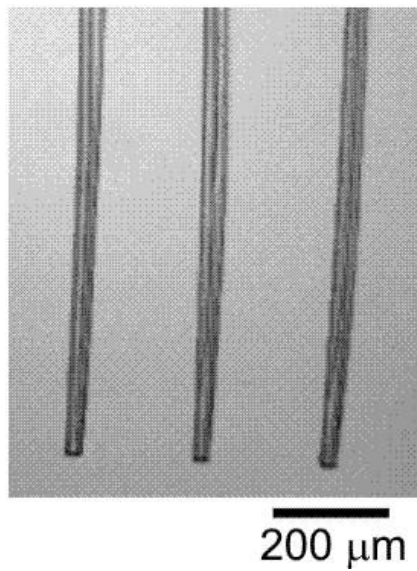

Figure R7. Folding of a freely floating polymer bilayer (a) and of a polymer bilayer placed on a substrate (b)

In other words, this is the case of a freely floating bilayer, where diffusion of water inside the hydrogel layer is not restricted by any substrate. Furthermore, it was confirmed that such freely floating bilayers undergo short-side rolling that is similar to the behavior of standard actuators (Figure R7a). As the studied bilayers are placed on a substrate, it is reasonable to assume that diffusion of water inside the active monolayer upon activation ( $\mathrm{T}$ $<$ LCST) occurs primarily through its lateral sides. Additionally, not only does the substrate hinder diffusion, it also exerts adhesion forces to the bottom surface of the bilayer that impede actuation until a certain threshold of detachment forces is reached. This means that bending, which requires detachment of the substrate, occurs only at a sufficient activation strain. In particular, non-swollen areas do not bend.

A more complicated scenario is observed when the width of the films is smaller and the length is considerably larger than the deformed circumference. The rolling itself starts first at the corners, as before, but longside rolling starts later (Figure R8b and Figure R5) and is energetically favored. Rolling along the short side is unfavorable because it implies more stored stretching energy along the long side. Further long-side rolling makes 
diagonally rolled corners unfavorable and leads to the switching of bent corners to a "long-side rolling" scenario.
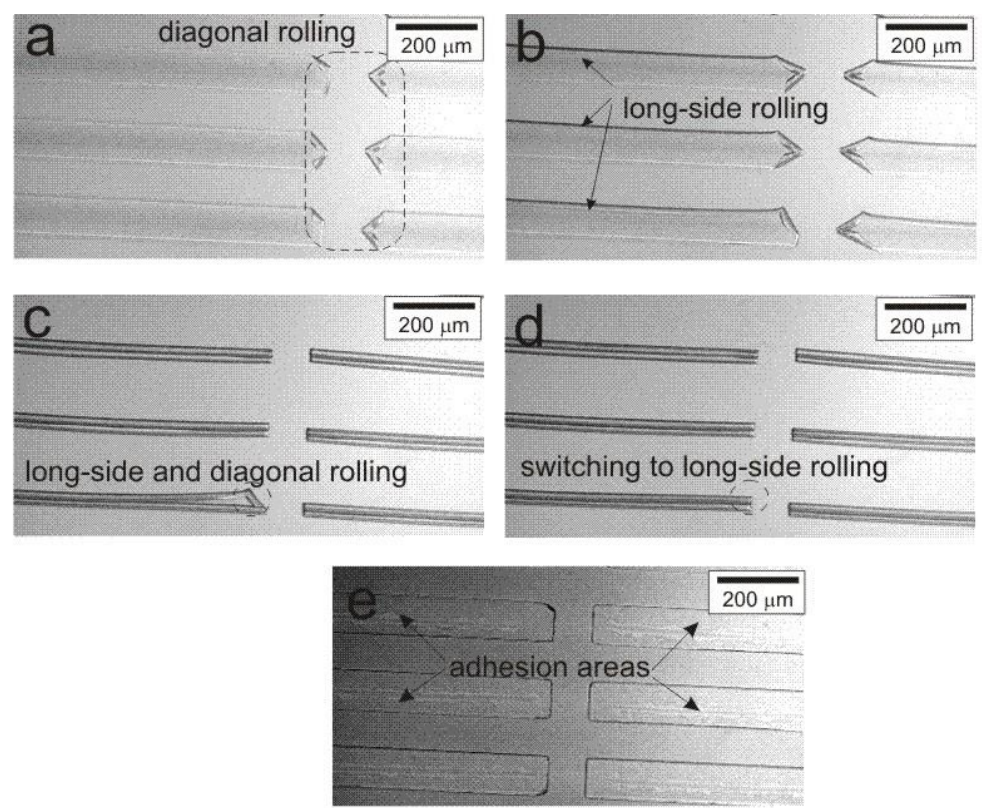

Figure R8. Folding of a rectangular polymer bilayer with aspect ratio 1/8. a) folding starts at the corners; b) tubes are formed along the long sides of the structures; c) and d) switching to long-side rolling; e) unfolded rectangles; the middle line denotes the adhesion area.

Depending on the width of the film compared to the deformed circumference, either an incompletely rolled tube is formed or the two longside rolling fronts collide into a completely rolled or doubled tube. If the deformed circumference is considerably smaller than the width and length of the films (which implies a high activation strain), then rolling starts first from corners and then continues along all sides (Figure 8c). The rolling fronts do not collide until several revolutions are made, which were shown to be almost irreversible. As a result, already rolled fronts are unable to unroll and irreversible all-side rolling is observed.

We investigated in detail folding of rectangular stimuli-responsive hydrogel-based polymer bilayers with different lengths, widths, and thicknesses located on a substrate. It was found that long-side rolling dominates at high aspect ratios (ratio of length to width) when the width is comparable to the circumference of the formed tubes. Moreover, rolling from all sides occurs when the width and length considerably exceed this 
circumference. Diagonal or all-side rolling is observed when the width and length are comparable to the circumference. On the other hand, short-side rolling was observed very rarely and in combination with diagonal rolling. ${ }^{161}$

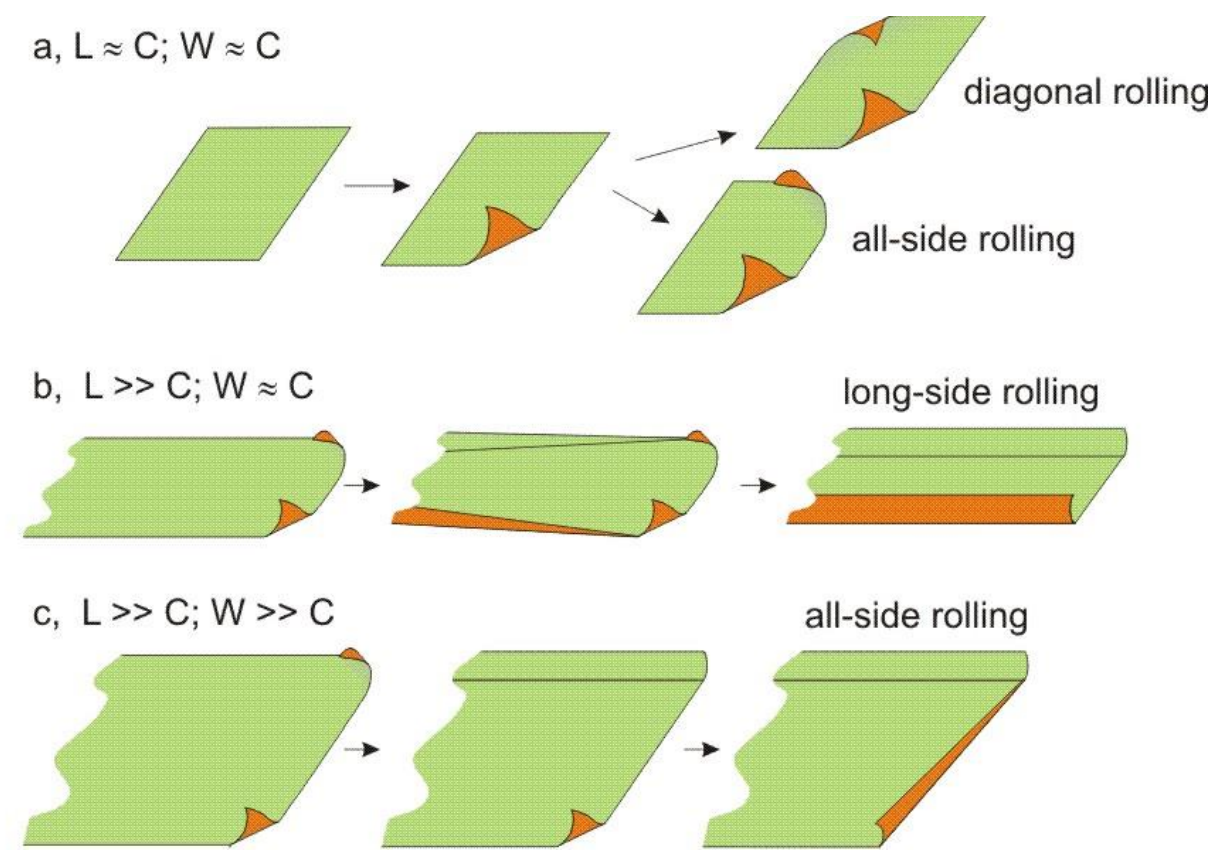

Figure R9. Summary of the folding behavior of polymer bilayers, placed on a substrate

\section{Summary}

On the basis of the experimental observations it was argued that bilayers placed on a substrate start to roll from corners due to quicker diffusion of water. Rolling from the long-side starts later but dominates at high aspect ratio due to energetic considerations. We have shown experimentally and confirmed by theoretical considerations that the main reasons causing a variety of rolling scenarios are (i) non-homogenous swelling due to slow diffusion of water in hydrogels and (ii) adhesion of the polymer to a substrate until a certain threshold. Moreover, non-homogenous swelling determines folding in the first moments, while adhesion plays a decisive role at the later stages of folding. The films that we investigated are fabricated on the microscale. On the other hand, the knowledge obtained in this work is applicable to thinner films to direct their folding in order to form tubes with diameters in the nano-range. 


\section{Folding of Complex Shapes. One-Step Folding}

\section{Motivation}

It was shown in the previous chapter that one can control the diameter of folding by adjusting the thicknesses of the polymer layers. Different folding scenarios arising in rectangles with altering width-to-length ratios were also demonstrated. Thus, a rectangle could provide us with the possibility to design and produce only one regular 3D structure - a tube. Our goal, however, was to explore the possibilities of designing and creating more sophisticated structures. First, we decided to make self-folding capsules. Selffolding capsules have promising potential applications as transportation vessels in microfluidics and drug delivery. For this purpose, four- and six-arm stars were chosen as template structures.

By adjusting the radius of folding, one can have the rays of the stars bending toward the center of the form and closing there, thus forming a sealed capsule. The general idea was to use a polymer bilayer, where one of the components was a biocompatible hydrophobic polymer and the other was a thermoresponsive hydrogel. Biocompatibility was an important point, as the encapsulation of cells in the produced structures was planned. PMMA, which was used to investigate the folding behavior of rectangular polymer bilayers, was considered unsuitable for the upper layer due to one major drawback: it is stiff at room temperature. This property of PMMA (stiffness at ambient conditions) means that once folded, the structures are only capable of slow and partial unfolding. The goal was, however, to demonstrate quick and reversible encapsulation of cells. Therefore, polycaprolactone ( $\mathrm{PCl}$ ) was chosen for the passive layer, as it is commercially available, cheap, biocompatible and biodegradable; it easily forms homogeneous layers when deposited from solution; it has a $\mathrm{T}_{\mathrm{g}}$ of around $60^{\circ} \mathrm{C}$ and is thus quite soft at room temperature, potentially allowing for multiple folding-unfolding cycles to be performed. A thermoresponsive polymer was essential, as it makes it easy to pack cells in a structure: the cells can be first deposited on the surface of the polymer blend that, after applying an external stimulus, would form a 
capsule. Therefore, PNIPAM was chosen for the lower layer. PNIPAM is a well-known and widely used polymer with an LCST of $33^{\circ} \mathrm{C}$, i.e. it is soluble at temperatures lower than $33^{\circ} \mathrm{C}$ and loses its solubility above this temperature.

Photolithography was used for the preparation of the stars, so the polymers had to be able to cross-link under UV light. For this reason, a random co-polymer of PNIPAM with benzophenone acrylate was synthesized. PCl was purchased from a commercial source and could not crosslink. To overcome this problem, 4-hydroxybenzophenone was added to the $\mathrm{PCl}$ solution prior to depositing, as it forms radicals under the irradiation with UV light and can thus crosslink the polymer.

\section{Experimental part}

Synthesis of poly-(NIPAM-BA): BA (0.02253 g, $0.089 \mathrm{mmol})$, NIPAM (1 g, $8.85 \mathrm{mmol})$, AIBN (0.01453 g, $0.089 \mathrm{mmol})$ were added in a $10 \mathrm{ml}$ test tube. The components were dissolved in $6 \mathrm{ml} \mathrm{1,4-dioxane} \mathrm{and} \mathrm{degassed} \mathrm{with}$ nitrogen for $30 \mathrm{~min}$. The test tube was tightly sealed and placed into a shaker $\left(70{ }^{\circ} \mathrm{C}, 90 \mathrm{rpm}\right)$ for $24 \mathrm{~h}$. Then the mixture was cooled down to room temperature and poured slowly into diethyl ether under vigorous stirring. The precipitate was filtered and dried under vacuum. The poly(NIPAM-BA) had a slightly lower cloud point $\left(\mathrm{T}=28^{\circ} \mathrm{C}\right)$ than the PNIPAM homopolymer $\left(\mathrm{T}=33^{\circ} \mathrm{C}\right)$.

Preparation of the solutions: An 8\% solution of P(NIPAM-BA) in ethanol was prepared. The solution was filtered through a $0.2 \mu \mathrm{m}$ PTFE filter prior to use. Three solutions of $\mathrm{PCl}$ in toluene with different concentrations were prepared: $3 \%, 5 \%$ and $8 \%$. 4-hydroxybenzophenone was added to each of them, its concentration being 6 mole\% relative to the concentration of $\mathrm{PCl}$. Due to their high viscosity the solutions were not filtered and were used "as is". 


\section{Results and discussion}

As the introduction of a hydrophobic monomer in a co-polymer decreases its LCST, we first had to determine the cloud point of the $\mathrm{P}$ (NIPAM-BA). For that purpose, a $1 \mathrm{mg} / \mathrm{ml}$ solution of $\mathrm{P}($ NIPAM-BA) in water was prepared. The solution was then brought to $15^{\circ} \mathrm{C}$ and heated up to $35^{\circ} \mathrm{C}$ at a rate of approximately 0.2 degrees/sec. The LCST of the polymer was then settled visually by the temperature at which the polymer loses its solubility and forms a precipitate. It was determined that the P(NIPAM-BA) had an LCST of $28^{\circ} \mathrm{C}$.

Next, the thicknesses of the films and the radius of folding of the polymer bilayers prepared from the $\mathrm{P}(\mathrm{NIPAM}-\mathrm{BA})$ and different $\mathrm{PCl}$ solutions were examined. Homogeneous films with an average thickness of $4 \mu \mathrm{m}$ were obtained by manual dip-coating in P(NIPAM-BA). For the $\mathrm{PCl}$ solutions, manual dip-coating resulted in films with $2 \mu \mathrm{m}, 4 \mu \mathrm{m}$ and $8 \mu \mathrm{m}$ thicknesses, respectively. It was found that the $8 \mu \mathrm{m}$ blend detached from the surface due to swelling during the development process, making it impossible to create any structures. Due to that reason, the other two solutions were used. When combined with the $4 \mu \mathrm{m}$ P(NIPAM-BA) layer, they resulted in a folding diameter of $100 \mu \mathrm{m}$ and $50 \mu \mathrm{m}$ for the $5 \%$ and the $3 \%$ solution, respectively. These radii of folding suited us well, as the stars had arms approximately 100 $\mu \mathrm{m}$ in length. 


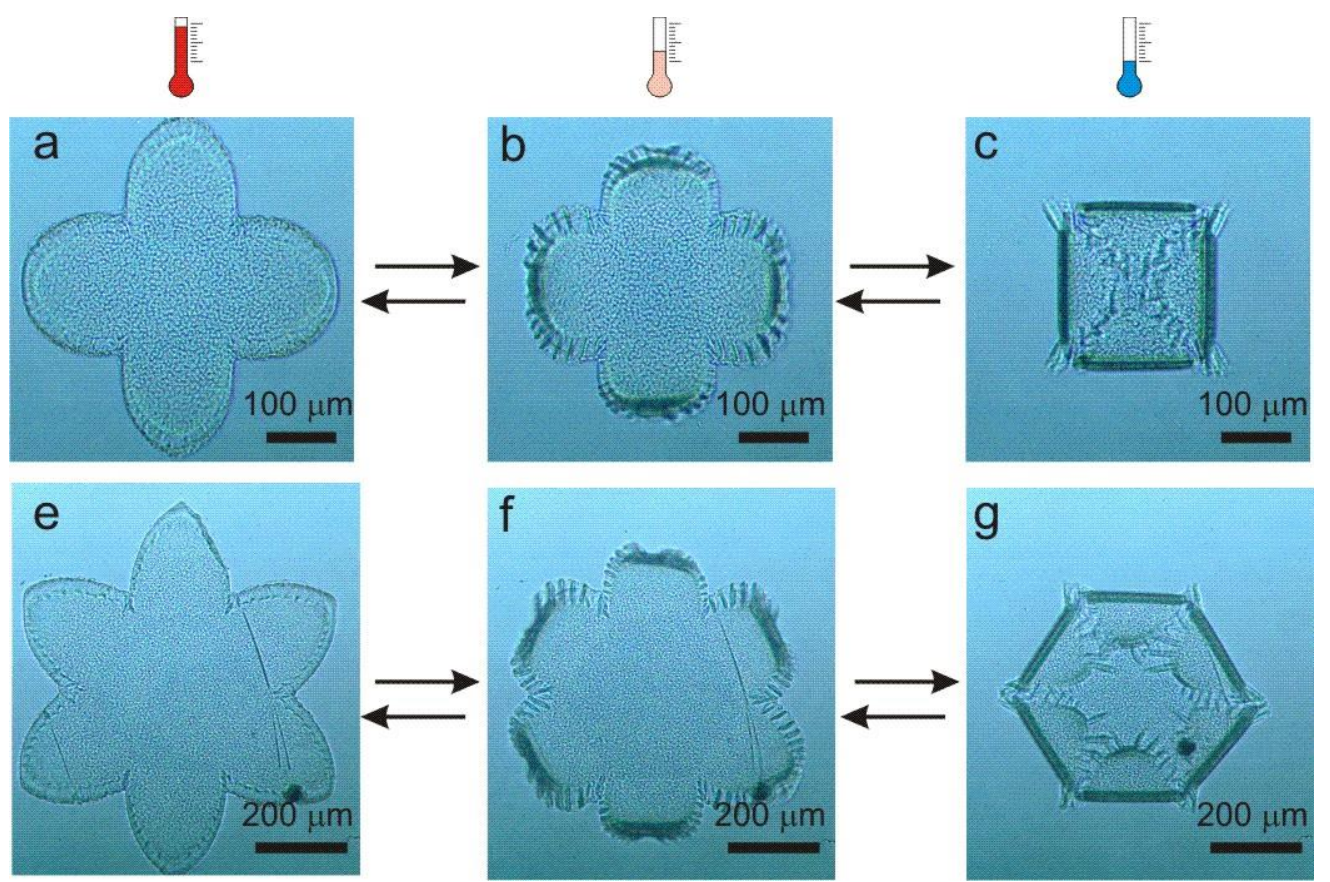

Figure R10. Bright field optical microscopy images of self-folding of star-like patterned polymer bilayers. The polymer bilayer is undeformed at elevated temperature, when the poly-(NIPAM-BA) layer is shrunk. Cooling results in swelling of the poly(NIPAM-BA) layer and folding of the bilayer.

Next, folding of the patterned bilayers in aqueous environment was investigated. It was found that star-like bilayer films remain undeformed at $\mathrm{T}$ $>28^{\circ} \mathrm{C}$ (Fig. R10 a,e). The arms of the bilayer started to bend when the temperature decreased below the cloud point of poly(NIPAM-BA) (Fig. R10 b,f). The bending was typically completed after 5-10 s. Experimentally it was found that $4 \mu \mathrm{m}$ thick poly(NIPAM-BA) and PCl layers allowed complete and partial folding of the four-arm and six-arm stars, respectively. (Fig. R10 c,g). Using of a thinner $\mathrm{PCl}$ layer led to a smaller curvature radius, multiple rolling of the arms and incomplete folding of the capsules. A similar effect was observed with a thicker PCl layer when the temperature was reduced to $10^{\circ} \mathrm{C}$ or less (see fig R11). Lowering of the temperature led to stronger swelling of the active layer, thus decreasing the radius of folding. 


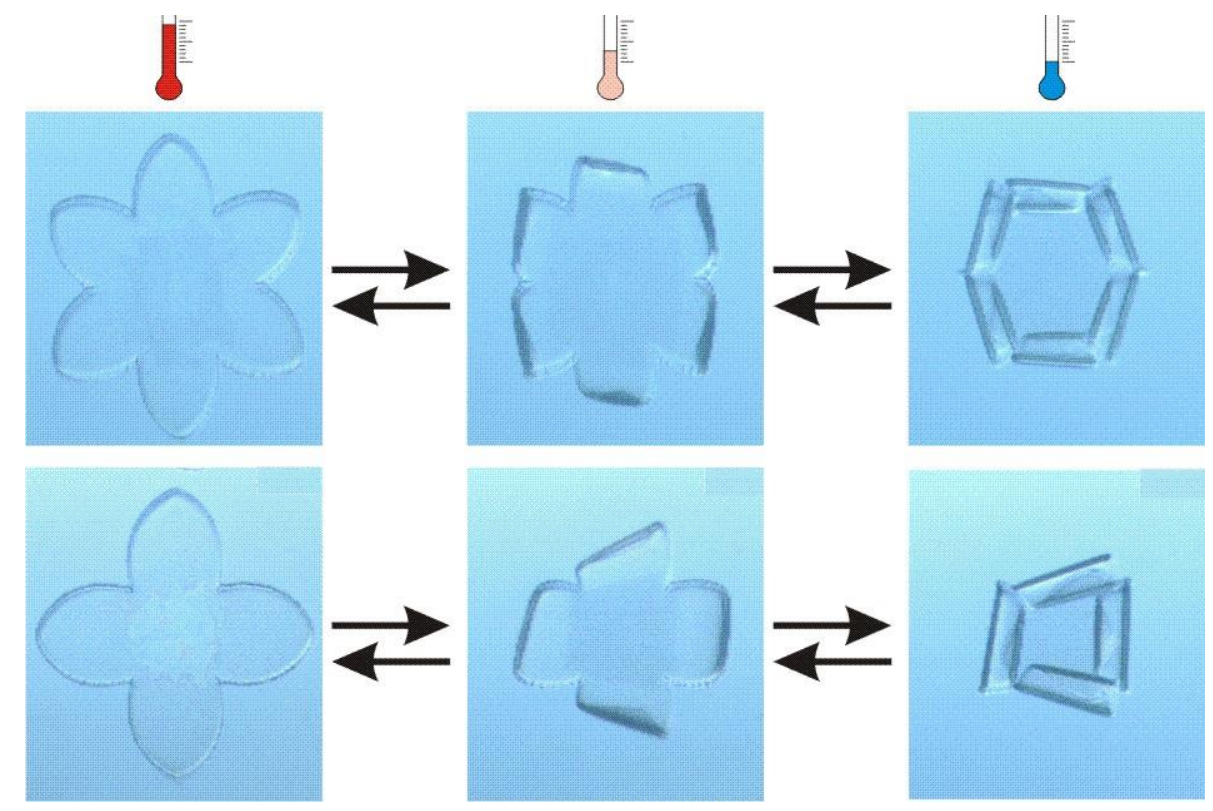

Figure R11. Bright field optical microscopy images of self-folding of star-like patterned polymer bilayers. The temperature range varies from $30^{\circ} \mathrm{C}$ (left) to $10^{\circ} \mathrm{C}$ (right).

The folded polymer layer stayed bound to the substrate because the centers of both four- and six-arm stars remained almost undeformed at moderately low temperature $\left(\mathrm{T}=20^{\circ} \mathrm{C}\right)$ for a long time. The capsules were released from the substrate after further cooling $\left(\mathrm{T}<10^{\circ} \mathrm{C}\right)$ due to strong swelling of the poly(NIPAM-BA) layer that resulted in a considerable deformation of the central part. Increasing the temperature led to a shrinking of the poly(NIPAM-BA) layer and unfolding of the capsules. Therefore, the unfolding was completely reversible and no residual deformation of the polymer bilayer was observed even after several folding-unfolding cycles.

As it was mentioned above, both 4- and 6-arm stars remained bound to the substrate even after several cycles of folding-unfolding because their centers remained almost undeformed at a moderately low temperature $\left(\mathrm{T}=20^{\circ} \mathrm{C}\right)$ for a long time. This is due to the fact that water diffuses much quicker through the ends of the star's rays than through the points of junction (see also "Folding of complex shapes. Multi-step folding"). Consequently, a "star" with only two rays, which will effectively have the shape of an ellipse, will detach much easier from the surface of the silicon wafer. Indeed, it was observed (fig R12). 


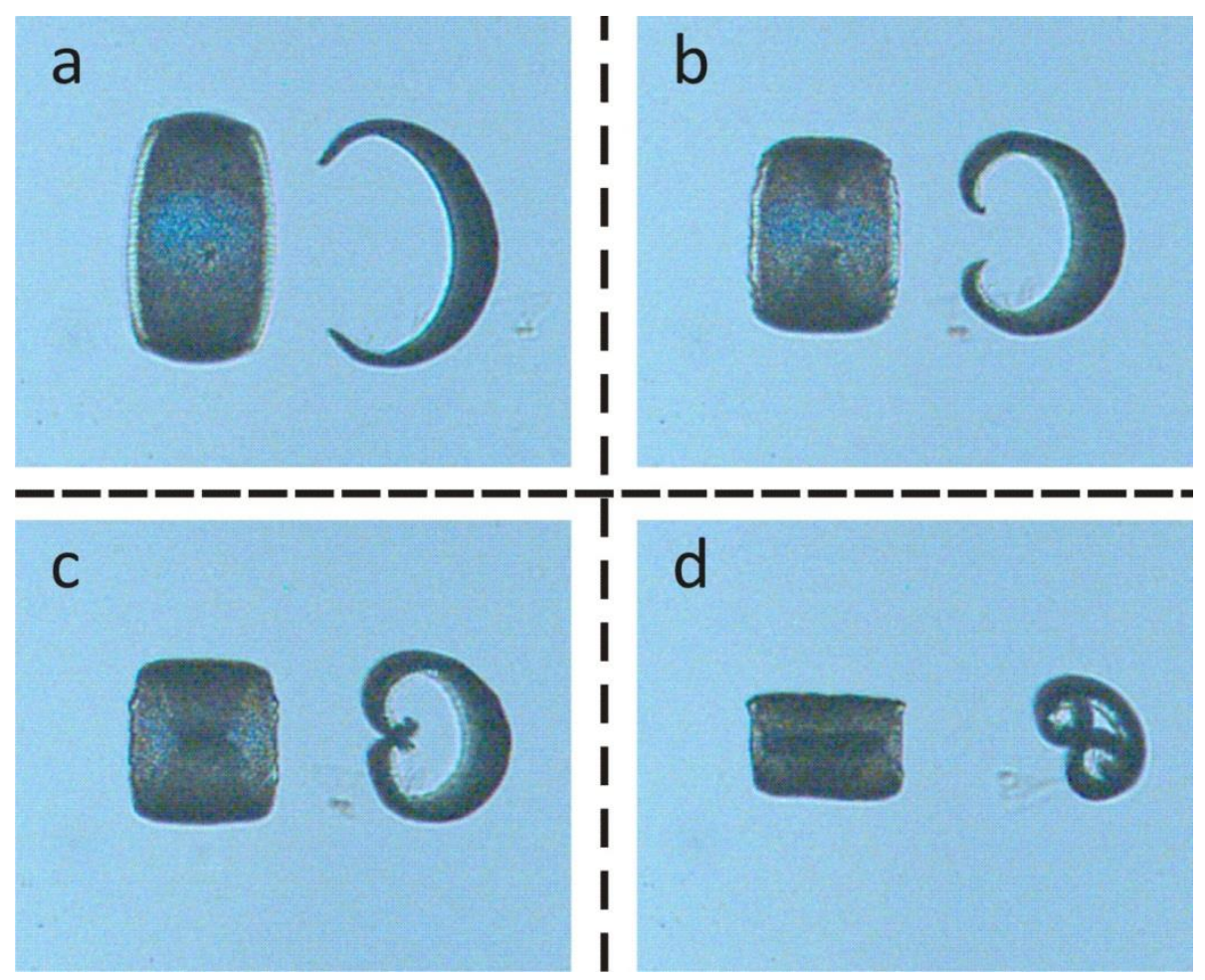

Figure R12. Folding of an elliptical ("two-arm star") polymer bilayer

As can be seen on the images, the attached and detached polymer bilayers exhibited the same folding behavior in terms of following the same bending path in an equal, time-resolved manner. This was in contrast to the case of rectangular blends, where the folding scenario strongly depended on whether a substrate was present or not (see the previous section). It can be easily explained if one considers the geometry of bilayers and the ratio between the diameter of folding and the size of the bilayers. Here, the circumference of folding is larger than the width of the ellipse. Hence, folding along the long axis is unfavorable even for a freely-floating polymer film. Therefore, the only possibility left is folding from the tips and towards the center. The same folding behavior, however, is to be expected for a bilayer on a substrate, as swelling and thus folding starts from the tips of the rays. Thereupon, in this particular case folding is independent of the presence of a substrate.

Finally, the possibility to encapsulate and release yeast cells using selffolding star-like polymer bilayers was demonstrated. 

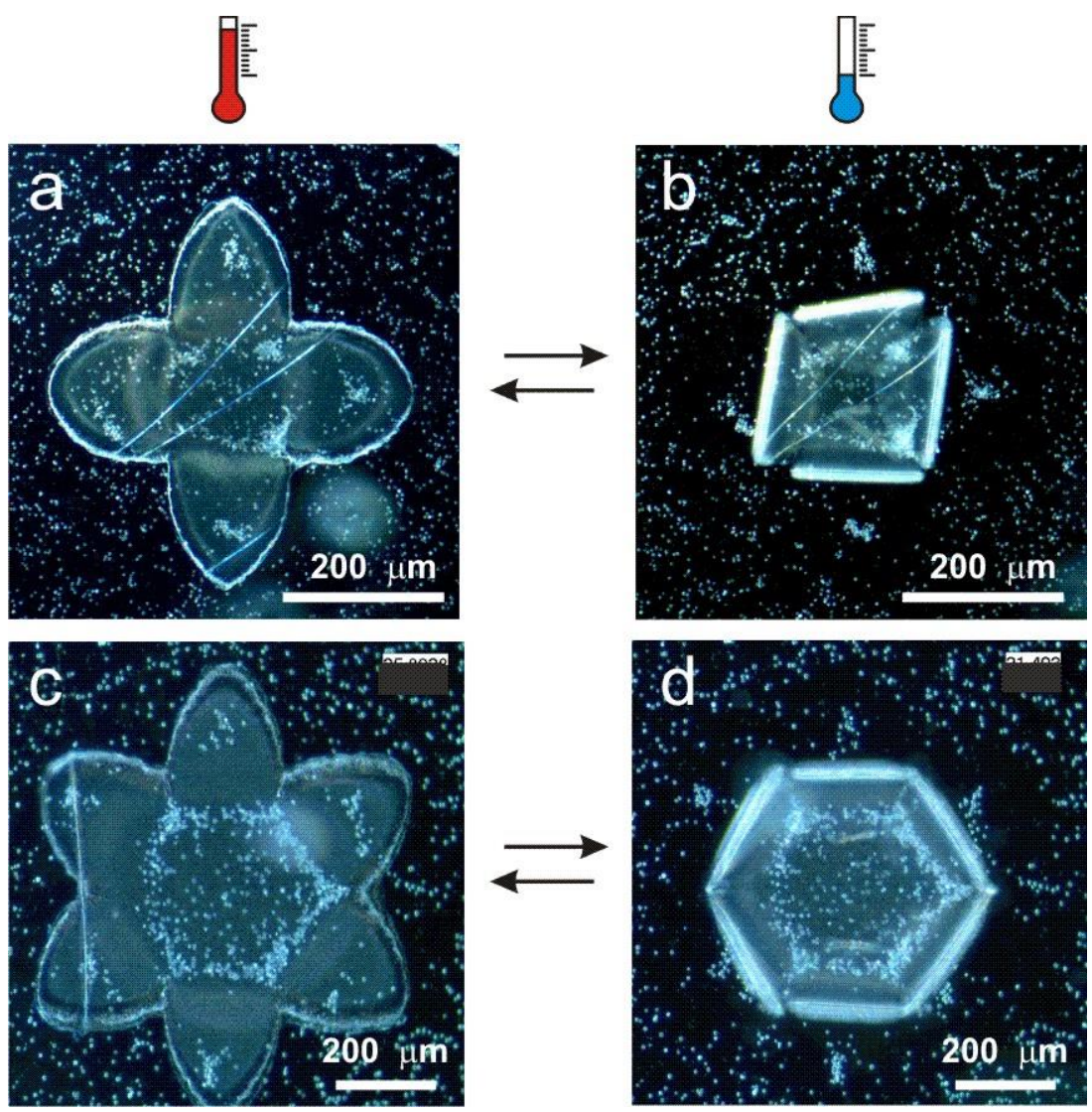

Figure R13. Encapsulation of yeast cells inside the thermoresponsive selffolding capsules (dark field optical microscopy). Yeast cells are adsorbed on the polymer bilayer at an elevated temperature. Cooling leads to swelling of the thermoresponsive polymer and folding of the capsules.

For this purpose, backing yeast cells were deposited on the patterned polymer bilayer from a buffer dispersion at an elevated temperature when poly(NIPAM-BA) is shrunk (Fig. R13 a,c). The surface density of the cells was in the range of 2000-10000 cells $/ \mathrm{mm}^{2}$ depending on the sample. A decrease in the temperature resulted in swelling of the poly(NIPAM-BA) and led to folding of the polymer capsules and encapsulation of the cells (Fig. R13 b,d). The yeast cells became accessible to the environment and could be removed from the polymer surface by liquid stream upon unfolding at an elevated temperature when the poly(NIPAM-BA) layer was shrunk. It was also observed that cells were concentrated in the center of the capsules after several folding cycles, which was caused by their sliding from the arms during folding. Consequently, these experiments demonstrated the possibility for encapsulation and release of cells using self-folding capsules. 
All the shapes presented in this section so far were designed by dividing a sphere by regularly spaced cuts going from one of its poles without reaching the other one and subsequent flattening of the opened sphere. The main idea behind this design was to obtain spherical, or sphere-like, closed particles after folding of the polymer bilayer. As can be seen from Figure R14, this is exactly the outcome in the case of a 4 -arm star. A 6 -arm star forms, however, a doughnut-like capsule, which is due to the fact that the rays of the star are too short in order for their tips to meet at the center of the figure. Therefore, an alternative design of a six-arm star, which had longer rays and a smaller middle part, was suggested (see Figure R14a).

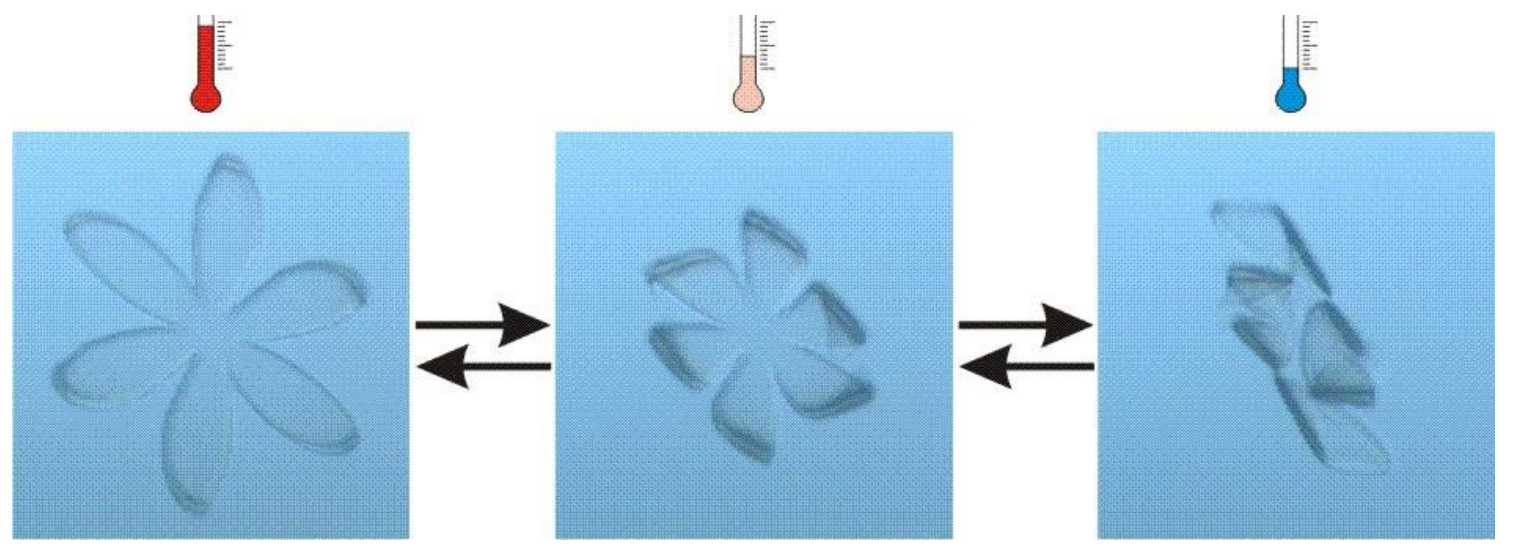

Figure R14. Folding of a 6-arm star with decreased ray width

However, this form failed to form a closed capsule. As can be seen from Figure R14b, the rays of the star did not fold precisely towards the center of the bilayer. Moreover, some of the arms folded slightly slower than others, which added further distortions in its final shape. Figure R14c represents the typical overall outcome of the process. 162

\section{Summary}

In conclusion, we demonstrated the design of partially biodegradable thermoresponsive self-folding capsules capable of controlled capture and release of cells. The proof of principle was demonstrated on the example of star-like patterned polycaprolactone-poly(N-isopropylacrylamide) bilayers, which reversibly encapsulate/release yeast cells in response to a temperature signal. 


\section{Folding of Complex Shapes. Multi-Step Folding}

\section{Motivation}

The folding behavior of rectangular and star-shaped polymer bilayers was investigated so far. Different folding scenarios arising in rectangles with altering width-to-length ratios were also demonstrated. A rectangle, though, could only provide us with the possibility to design and produce only one regular 3D structure - a tube. After adjusting the radius of folding, the starlike patterns yielded capsules, which were able to reversibly encapsulate cells and, potentially, other micro- and nano-objects. Noteworthy, these capsules were produced by means of the same process we used for the design of the tubes - when compared to the folding of rectangles, it was the shape of the initial pattern and the folding radius that were changed; the mechanism was the same - simple one-step folding towards the center of the bilayer. It is clear that the number of structures that can be generated by this method is fairly limited. However, other systems are able to produce much more sophisticated shapes, like cubes, pyramids, dodecahedrons. One reason for this is that all the other systems include complex and expensive methods like, for example, multi-step photolithography. This grants the introduction of hinges or other inhomogeneities in the structure, thus providing additional means of control by allowing folding to occur in a certain, pre-defined direction. On the other hand, polymer bilayers produced by our method are homogeneous. The parameters we control are the circumference of folding (by adjusting the thicknesses of the layers) and the shape and size of the structures (by applying different photomasks). Due to the isotropy of the mechanical properties of the bilayer, the formed structures are hinge-free and have rounded shapes. Nevertheless, folding in nature can have a very complex character, which strongly depends on the geometry and swelling path that may result in multistep folding (development of curvature in different directions). Gaining inspiration from biological systems, we demonstrate that the shape of isotropic polymer bilayers is able to direct folding in a sophisticated manner leading to even more complex hierarchical 
folding than in nature. In particular, films can undergo sequential folding steps by forming various 3D shapes with sharp hinges. By analyzing the folding patterns we elucidated empirical rules, which allow the folding to be directed, leading to the design of specific 3D shapes.

\section{Experimental part}

Synthesis of P(NIPAM-AA-BA): BA (0.28 g, 1,12 mmol), NIPAM (6 g, $51.57 \mathrm{mmol})$, AA (0.2556 g, $3.36 \mathrm{mmol})$, AIBN (0.01632 g, 0. $38 \mathrm{mmmol})$ were added in a $50 \mathrm{ml}$ flask. The components were dissolved in $30 \mathrm{ml}$ ethanol and degassed with nitrogen for $30 \mathrm{~min}$. The polymerization was carried out overnight at $70{ }^{\circ} \mathrm{C}$ under continuous stirring. After cooling, the mixture was poured in $750 \mathrm{ml}$ diethyl ether; the precipitate was filtered and dried under vacuum at $40^{\circ} \mathrm{C}$.

Synthesis of P(MMA-BA): 6.3 g MMA (62.7 mmol), 0.24 g BA $(0.96$ $\mathrm{mmol})$ and $0.05 \mathrm{~g}$ AIBN $(0.31 \mathrm{mmol})$ were dissolved in $30 \mathrm{ml}$ of toluene. The mixture was purged with nitrogen for $30 \mathrm{~min}$. The polymerization was carried out overnight at $70{ }^{\circ} \mathrm{C}$ under nitrogen atmosphere with mechanical stirring. After cooling, the mixture was poured in $750 \mathrm{ml}$ diethyl ether; the precipitate was filtered and dried under vacuum at $40^{\circ} \mathrm{C}$.

\section{Results and discussion}

For the experiments we used polymer films consisting of two layers of photo-crosslinked polymers: the active layer being a random thermoresponsive copolymer P(NIPAM-AA) and the passive layer being PMMA. The bilayer is located on a silica wafer in such a way that the active and passive polymers are the bottom and top layers, respectively. The bilayer is undeformed in PBS $0.1 \mathrm{M} \mathrm{pH}=7.4$ environment at $\mathrm{T}>70{ }^{\circ} \mathrm{C}$ and folding occurs after cooling below $70{ }^{\circ} \mathrm{C}$. Due to the relatively slow diffusion rate of water inside the $\mathrm{P}$ (NIPAM-AA) layer, actuation is driven by the progression of the diffusion front, along which the hydrogel starts to swell. This induces a path-dependency in the folding pattern, as the bilayer is not homogeneously activated, but progressively swells as water diffuses from the lateral sides. 
Investigations of the swelling were performed in a qualitative manner by observing the color change of the films, which reflects the change in optical path length (OPL) due to light interference (Figure R15). The OPL varies as a function of the film thickness and refractive index, which in turn depends on the swelling degree.

circle/ellipse

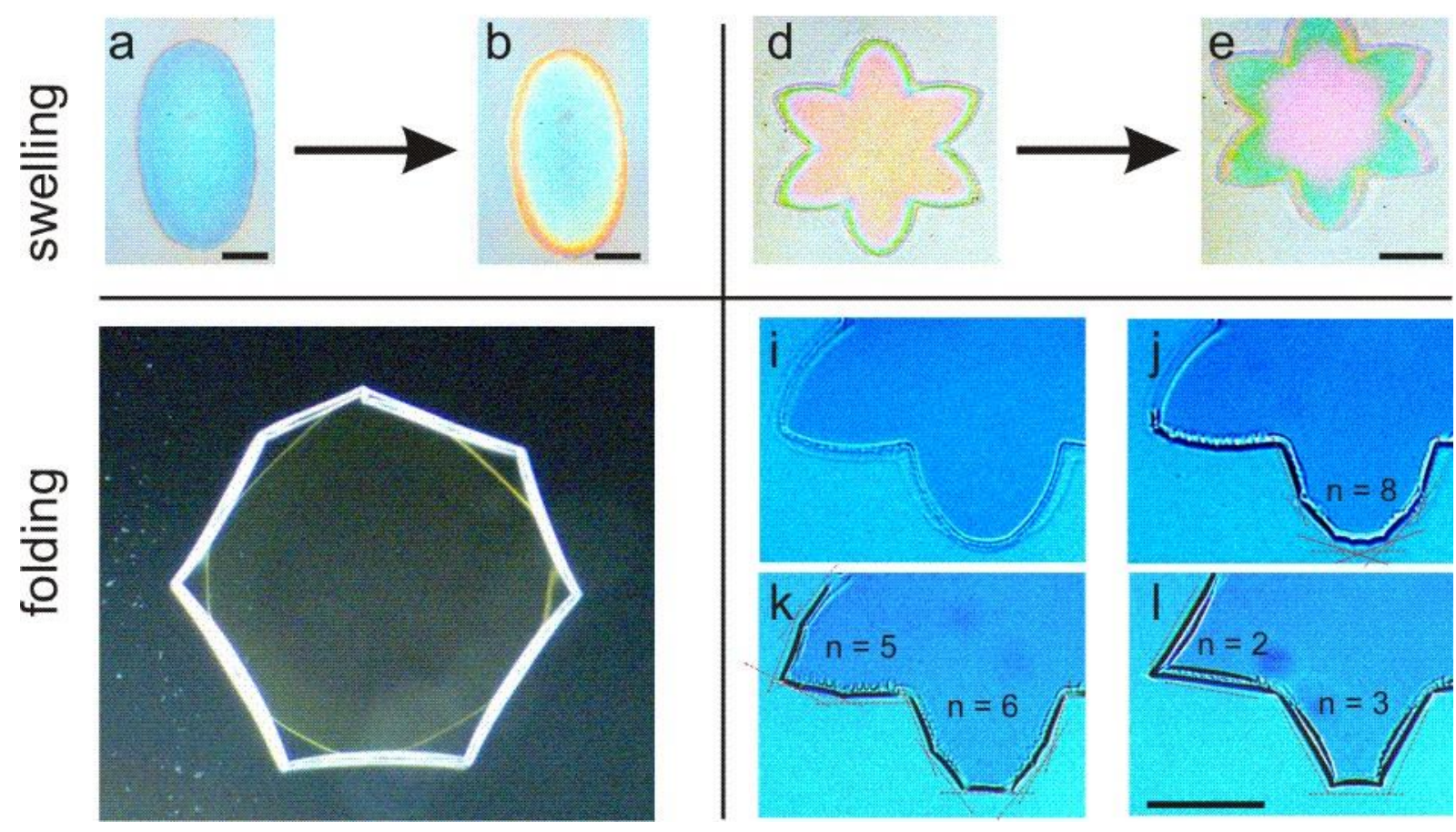

Figure R15. Swelling (upper panels) and first step of folding (lower panels) of circularlelliptical (left panels) and star-like (right panels) bilayer polymer films

The non-swollen elliptical and star-like films have a homogenous blue (Figure R15a) and reddish (Figure R15d) color, respectively. The difference in the color of both films is caused by their different starting thicknesses (Figure R15). The color of the films starts to change immediately after immersion in water at $25{ }^{\circ} \mathrm{C}$, with the elliptical film becoming redder while the star-like film becomes green (Figure R15b,e). The changes of color in both cases start from the outer periphery of the bilayer film. As the active layer is confined between a water-impermeable silicon wafer and hydrophobic PMMA, this suggests that water can only penetrate inside the layer from the lateral sides. The depth of water penetration along the perimeter of the film (activation 
depth) is uniform in both cases in the first moments of swelling. The differences in the swelling behavior between the two shapes appear after several seconds of incubation in water. The activation pattern depends on the external shape of the bilayers, with the position of the diffusion front (the activation depth) depending on the distance to the tissue border. This can be seen clearly in the differences of the activation patterns in the convex shapes like ellipses (Figure R15b), and concave ones like stars (Figure R15d). For star-like bilayers, the tips of the triangular-like arms swell faster than their base and their polygonal central part. This can be explained by the fact that after a certain time the diffusion fronts on either side of the arms intersect resulting in faster swelling. The experimental results show that the swelling starts from the periphery of the films and that the activation profile strongly depends on the shape of the film.

Next, we experimentally investigated the folding of circular/elliptical and star-like films. Modeling predicts that multiple wrinkles are formed along the perimeter of the folding bilayer when it is edge-activated. The spatial wavelength of the wrinkles is proportional to the activation depth (d) as observed in the wrinkles of leafs due to excessive radial edge growth ${ }^{163}$ and solved analytically in the context of geometrically nonlinear elasticity ${ }^{164}$. As the activation depth increases, the number of wrinkles decreases as $P / d$, where $P$ is the perimeter of the shape. The fact that there is both a gradient in the radial (edge-activation) and the transversal direction (bilayer) results in a combination of wrinkling and bending, respectively. In full agreement with the modeling predictions, experimental results show that the number of wrinkles decreases during folding (Figure R15i-l). Due to the transversal bending effect, the wrinkles actually evolve into local partial tubes as the activation depth increases. The process of merging of wrinkles can clearly be seen on a straight edge (Figure R16). 


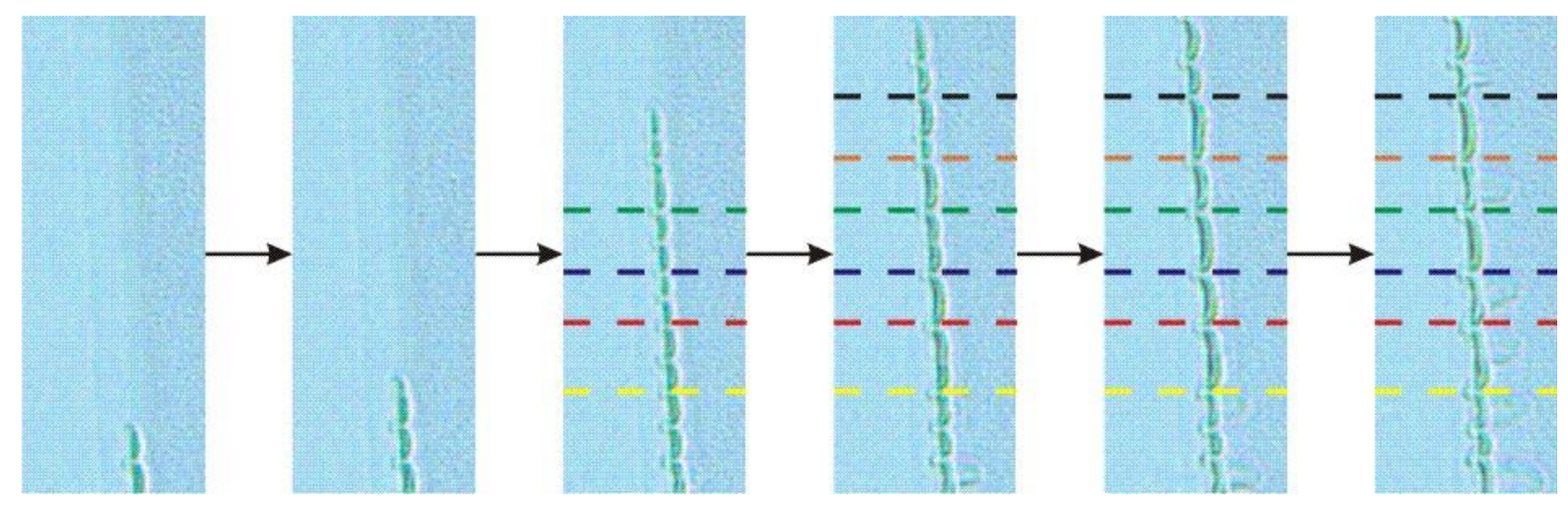

Figure R16. Evolution of wrinkling at the edge of a polymer bilayer during swelling. Wrinkles tend to merge after formation. Lines separate wrinkles that merged at some point during the process.

Here, wrinkling was induced by gradually immersing a rectangleshaped polymer bilayer in water. Swelling first started at one side of the rectangle and wrinkles were formed (Figure R16a,b). As the process continued, more wrinkles were formed (Figure R16c) and the already formed ones began to coalesce (Figure R16d,e,f).

For an indirect edge we observed that, at some point, the wrinkles stop to merge and their number remains constant. The probability of merging of two tubes depends on the angle ( 6 , Figure R15h) between them. Experimentally, we found that the critical value of $B$ below which merging of folded tubes was not observed is ca. $120-150^{\circ}$, which corresponds to $6-8$ wrinkles when starting with a circular shape (inset in Figure R15h). Based on these experimental observations we derived the first folding rule: "Bilayer polymer films placed on a substrate start to fold from their periphery and the number of formed wrinkles/tubes decreases until the angle between adjacent wrinkles/tubes approaches $130^{\circ}$. After the number of wrinkles/tubes along the perimeter of the bilayer film stopped to change the bilayers are locked for some time until the subsequent folding step occurs. For example, the wrinkled semi-ellipse bends towards its base (Figure R17a). 
a

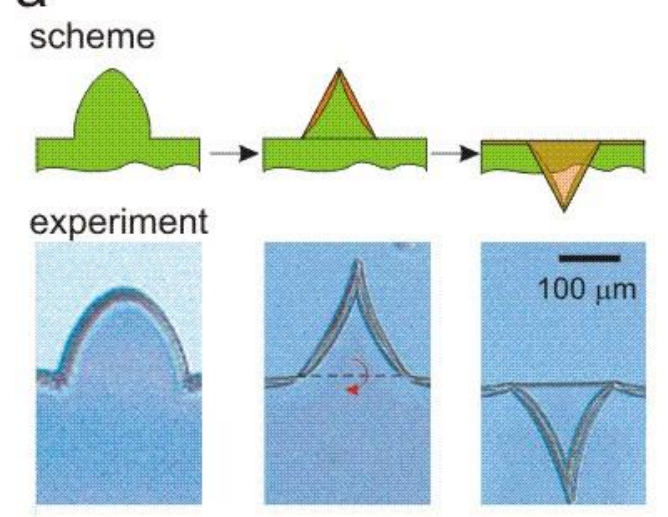

b

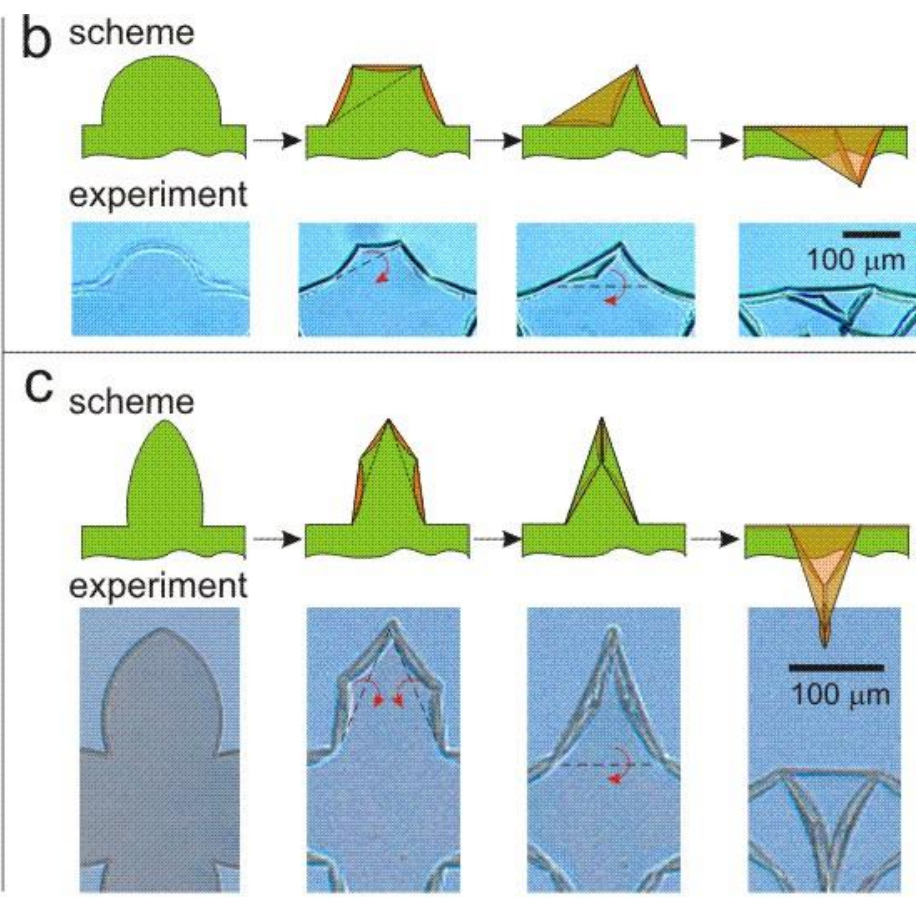

Figure R17. Schematic illustration, experimental observation, and modeling of the second step of folding of the elliptical arms depending on their shape

To explain the origin of the second step of folding we considered the geometry of the film after the first folding step. As mentioned, wrinkling of a bilayer leads to the formation of tubes along the perimeter of the film. Considering the fact that the rigidity of the tubes is higher than that of the undeformed films, the polygonal shapes are stiffened by this tube formation, and therefore possess a number of weak points located at the intersection of the tubes, i.e., at the vertices. These points act like hinges and folding is only observed along the lines connecting them (dashed line in Figure R17a). The formation of hinges during folding of isotropic bilayers, which to our knowledge has not been reported in the literature, is induced by the progressive activation from the lateral sides and the folded shapes are controlled by the initial shapes of the bilayers. This leads to the second rule of the folding: "After the wrinkles along the perimeter of the film form tubes, further folding proceeds along the lines connecting the vertexes of the folded film".

In case there are more than two hinges in the film, a question arises: upon which connecting line will the folding occur? The number of hinges is 
largely determined by the shape of the semi-ellipses. The regular semi-ellipse, which has a triangular shape after the first step of folding, simply bends toward the base along the line connecting the two bottom vertexes (dashed line in Figure R17a).
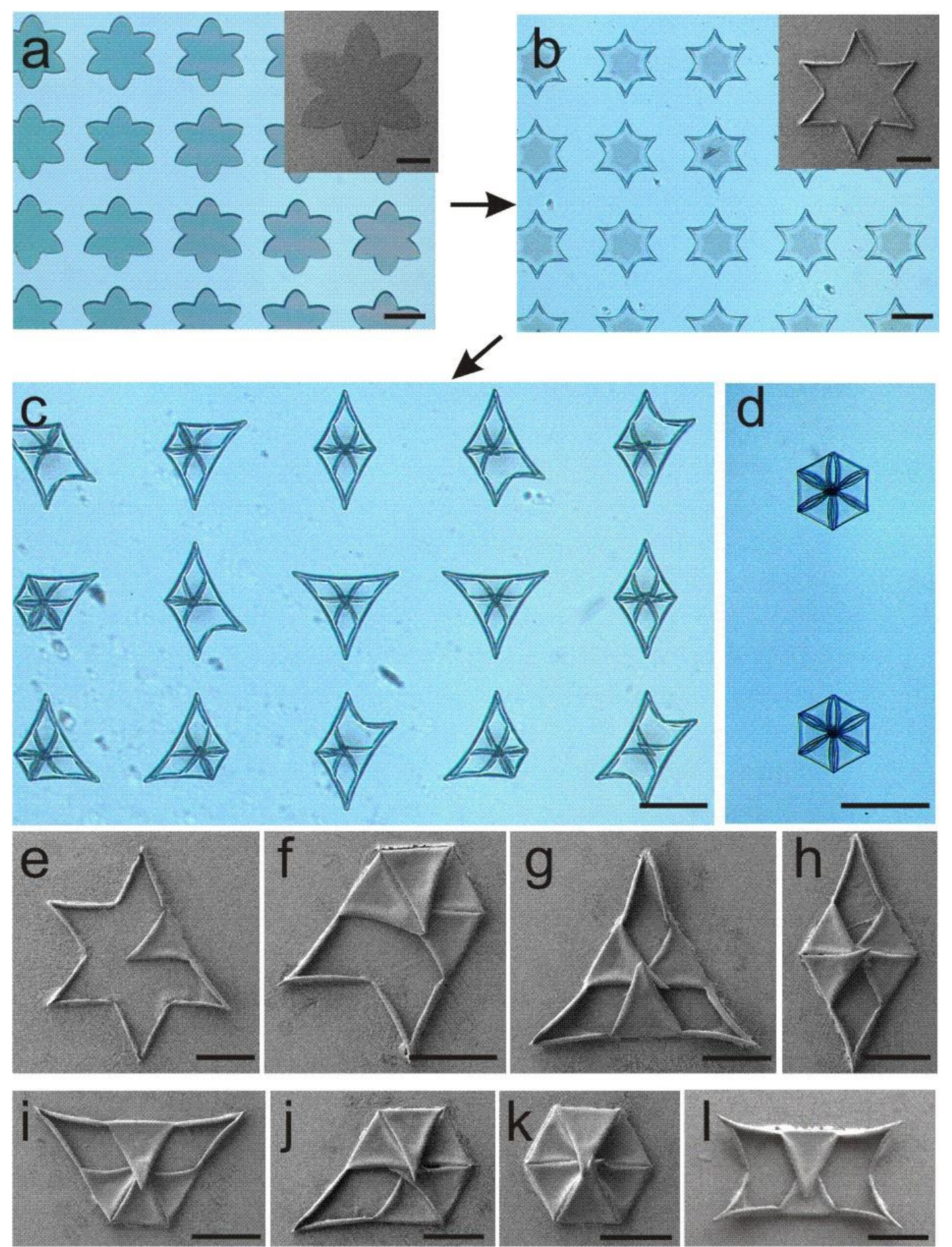

Fig R18. Examples of structures obtained by progressive edge-activation of six-ray star-like bilayers. a) Patterned bilayers; b) First step of actuation: wrinkles collapse into tubes; $c-1)$ Second step of actuation: rays fold leading to several configurations depending on the order of folding. Scale bars - $200 \mu \mathrm{m}$. 
If the semi-ellipse is more rounded, it forms a trapezoid after the firststep of folding (Figure R17b). In the second step of folding, the trapezoid bends along one of the lines connecting the opposite top and bottom vertexes (dashed line in the second image from the left in Figure R17b). Next, the formed triangle bends towards its base along the line connecting the two bottom vertexes. The elongated semi-ellipse forms four folds after the first step of folding (Figure R17c). Interestingly, the semi-ellipse folds further along the lines connecting the vertexes at the base and the top vertex and no folding along the lines connecting neither the vertexes of the middle nor the ones at the base is observed. Looking at the evolution of the activation pattern through time (diffusion profile see Figure R15), we observe that the lines connecting the hinges can only be used if they are within the activated pattern. Thus, the third rule of folding states: "the folding goes along the lines which are closer to the periphery of the films". Six-ray stars demonstrate the formation of very complex structures (Figure R18).

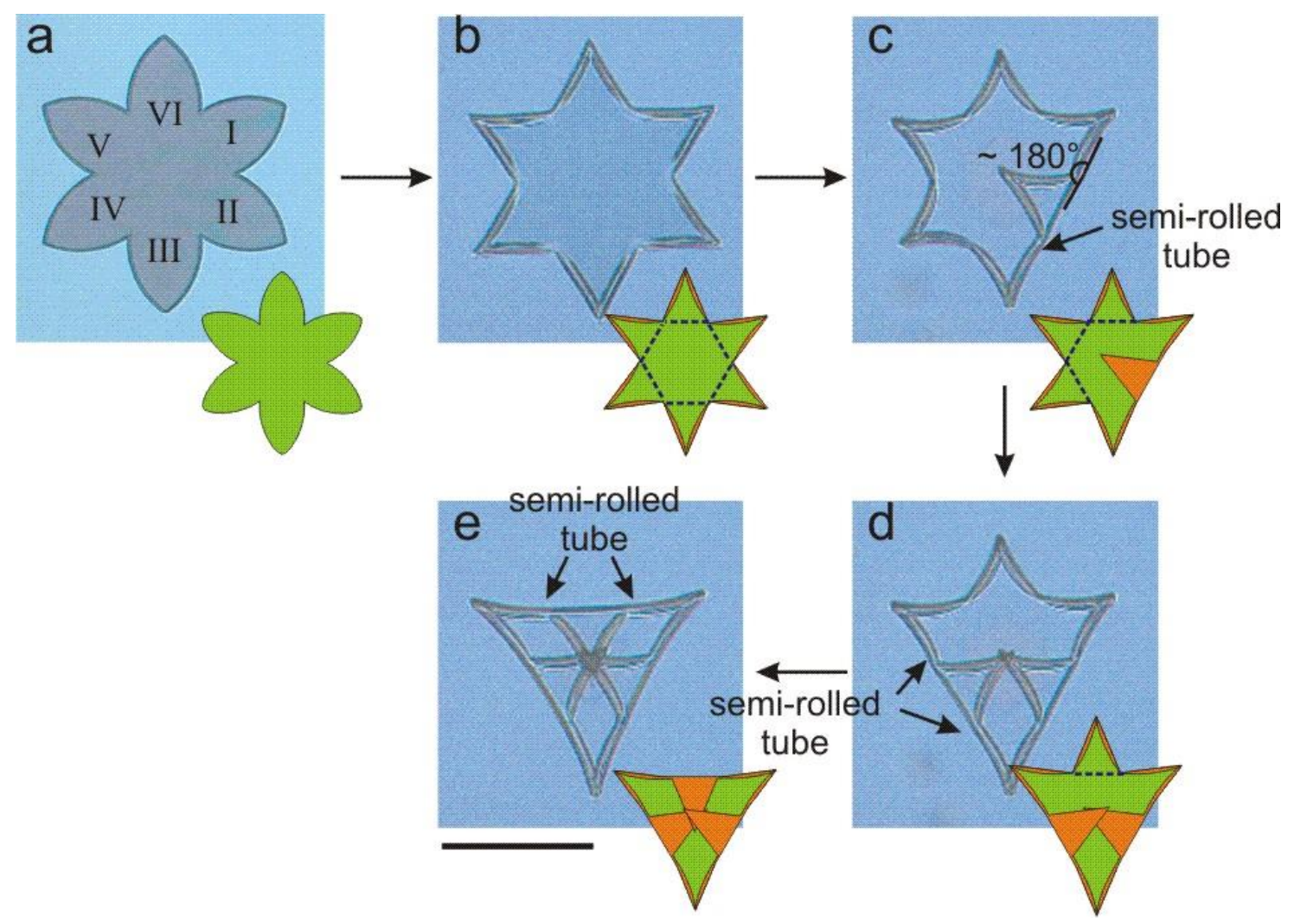

Figure R19. Microscopy snap-shots illustrating the mechanism of formation of triangles during actuation of a six-ray star. Scale bar is $200 \mu \mathrm{m}$. 
Notably, simultaneous folding of all rays is observed very rarely and in most cases triangles (Figure R18g) were formed. We investigated the folding in a time-resolved manner in order to explain the formation of the triangles (Figure R19).

Similar to the experiment demonstrated in Figure R15, wrinkles get longer and bend transversally into tubes (Figure R19b) thus increasing the rigidity of the ray. Next, one of the rays folds towards the center of the star (II in Figure R19c). Folding of this ray leads to the formation of a rigid semirolled tube, which is formed by the folded ray and the tubular shoulders of the adjacent rays (Figure R19c). The angle between the base of the folded ray and the shoulders of the neighboring arms is close to $180^{\circ}$ (Figure R19c). In this configuration, the weak points located at the intersection between I-II and II-III have disappeared and rays I and III (Figure R19c) cannot bend anymore. As a result, only three remaining rays (IV, V, VI) can bend. If ray $\mathrm{V}$ folds, no additional rays can bend (Figure R18l). If ray IV folds (Figure R19d) ray $\mathrm{V}$ is blocked. Finally, ray VI can fold leading to the formation of a triangle (Figure R19e). The discussed principle can be easily applied to understand the formation of the other observed figures (Figure R18c-1). In fact, several factors can be held responsible for the observed symmetry breaking (rays do not fold at the same time) such as inhomogeneities in the films and shape imperfections resulting in small deviations from the symmetric diffusion profile. Fig. R20 gives a summary of all possible structures that can be formed from a six-arm star.

Based on these experimental observations, one can derive the fourth folding rule: „Folding of the rays may result in blocking of the neighboring rays if the angle between the base of the folded ray and the shoulders of the neighboring rays is close to $180^{\circ}$. 

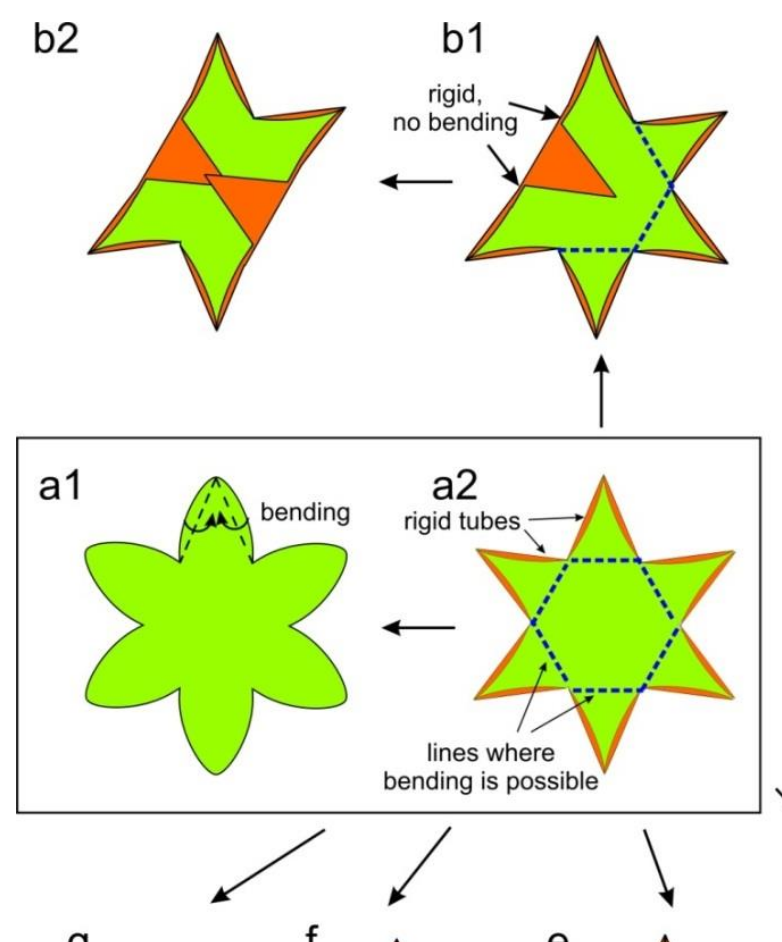

g

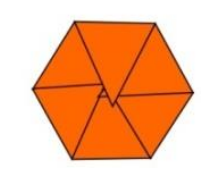

$\mathrm{f}$

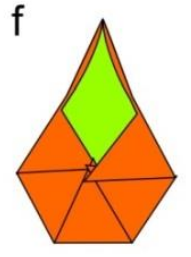

b1

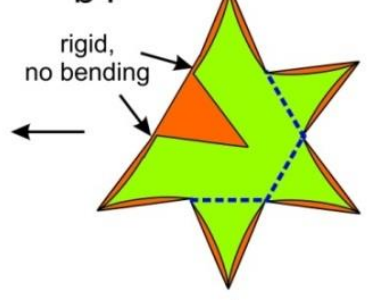

e

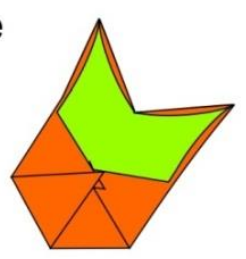

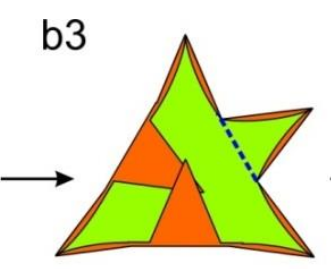

b4

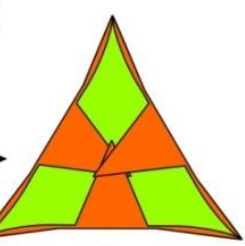

$\mathrm{c} 2$
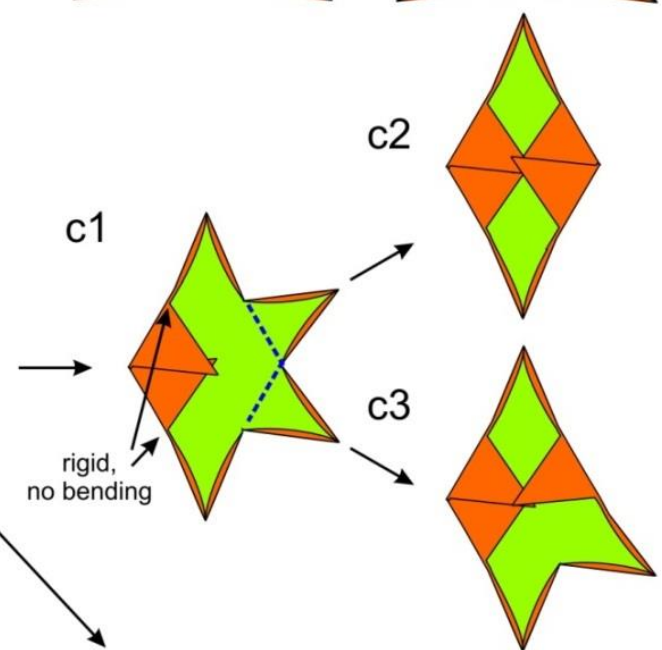

d1
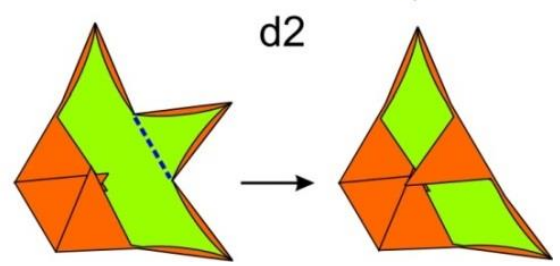

Figure R20. Summary of the outcomes of hierarchical multi-step folding of a 6 -arm star

Finally, we applied the derived rules for the design of truly $3 \mathrm{D}$ structures-pyramids. In fact, the reason why six-ray star formed flattened folded structures is their short arms and the hindering of folding of rays. Therefore, in order to fabricate pyramids we increased the relative length of the rays and changed the angle between them by decreasing their number (Figure R21a, b). The rays of the fabricated four-ray stars first wrinkle along their perimeter (Figure R21c, d). Four tubes are formed along the perimeter of each ray (first rule, Figure R21c), which then collapse two by two and form triangles (second rule, Figure R21d). Since the angle between the folds located on the shoulders of each ray is considerably smaller than $180^{\circ}$, the folding of rays is not self-interfering (forth rule) and all rays fold in the direction of the center of the star (third rule) thus forming a hollow pyramid (Figure R21e, f, g). 

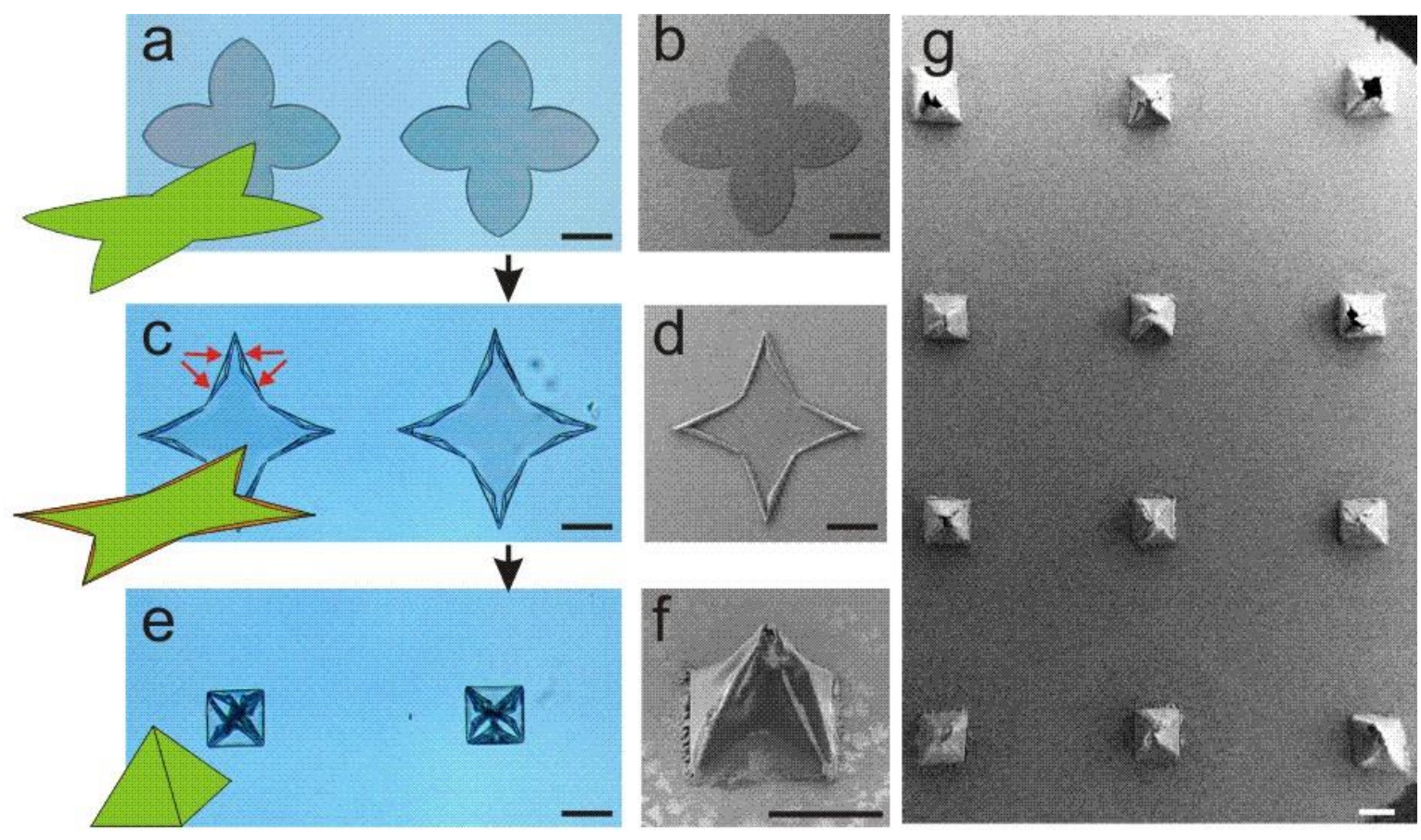

Figure R21. Sequential actuation of four-ray stars leads to the formation of pyramids. $a, b)$ unactivated film; $c, d)$ after wrinkling of the ray periphery into tubes; arrows indicate four wrinkles formed on each arm during first step of folding; e-g) after folding of rays leading to the formation of pyramids. Scale bar is $200 \mu \mathrm{m}$

As it was shown earlier, six-arm stars failed to form hexagonal pyramids because of two factors: first, the rays of the stars were too short and second, due to inhomogeneities in the polymer film, some arms started to fold later than other. The second problem, however, did not apply for four-arm stars because, in this case, if any two of the rays started folding at approximately the same time, they would eventually meat at the center of the structure and prevent each other from further folding. Hence, an almost $100 \%$ outcome of pyramids was obtained. A question arises: what outcome would a five-arm star have? To answer the question, we designed a five-arm star, the hierarchical folding of which should, at least theoretically, lead to the formation of a pentagonal pyramid. We used the same system as in the case of the four- and six-arm stars. We observed that only in few cases a pentagonal pyramid was formed, namely when four or all five rays started to bend simultaneously towards the center of the figure (Figure R22c). In all 
other cases, distorted pyramids, like those shown on Figure R22 a) and b), were obtained:
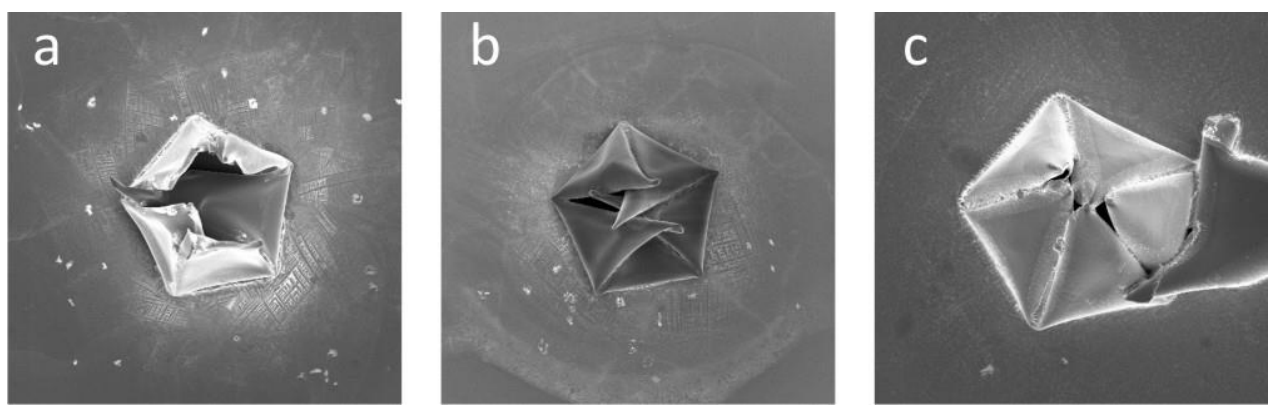

Figure R22. Examples of pentagonal pyramids, obtained by multi-step hierarchical folding; a) and b) distorted pyramids, suffering different types of folding errors; c) properly folded pentagonal pyramid.

The derived rules of folding are, in fact, applicable to other shapes, such as rectangles. As an example we included two-step folding of rectangles (Figure R23).
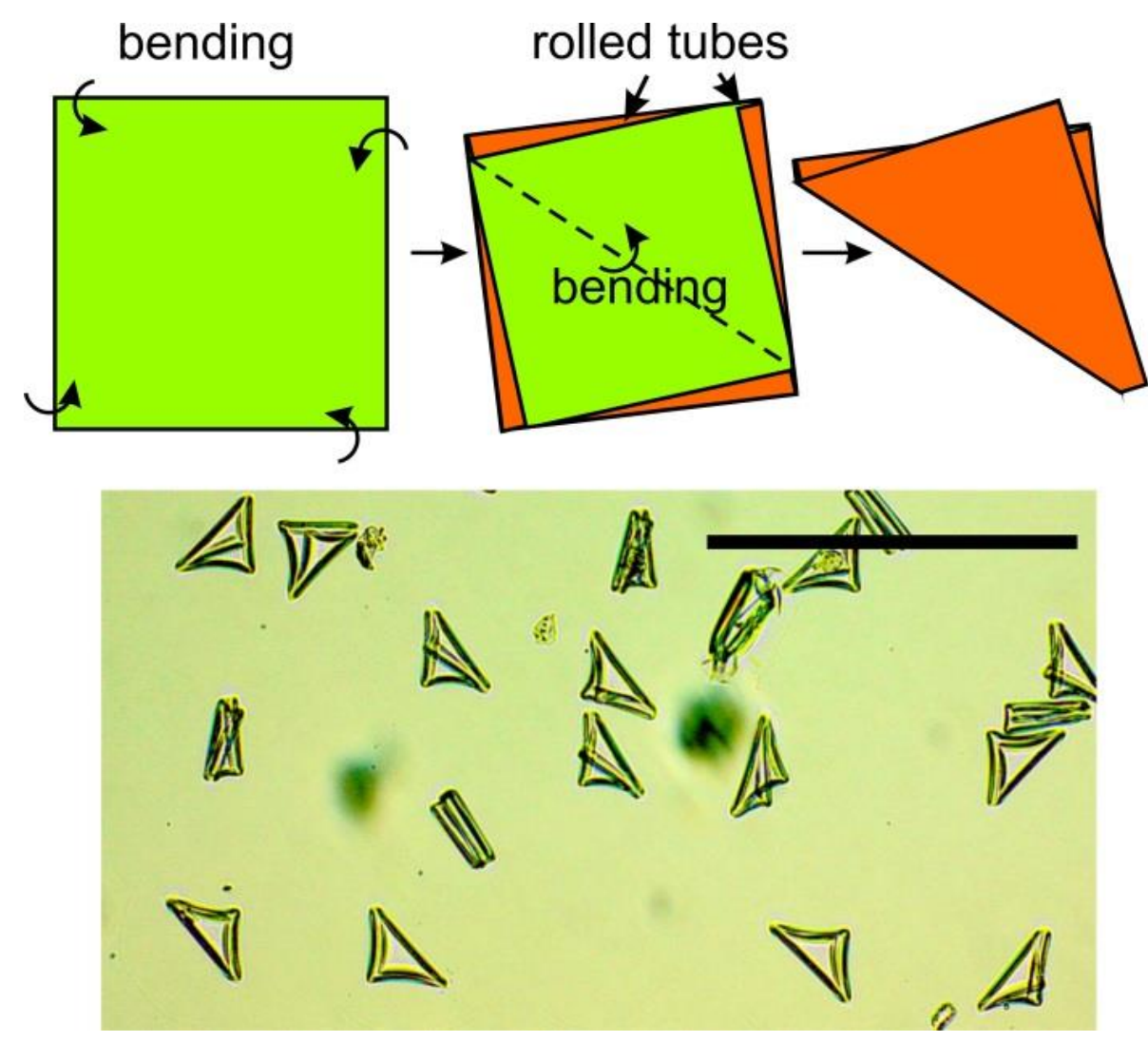

Figure R23. Two-step folding of rectangles. Scale bar is $500 \mu \mathrm{m}$.

We observed that, in general, the folding rules are applicable to all thicknesses. To argue that we performed many experiments with different 
thicknesses. The difference between thin and thick films was minor. For example, we observed that when star-like thin film fold, then all six arms (Figure R18d,k) can fold inside because the rigidity of the film is not sufficiently high. In the case of thick films, we typically observed folding of 34 arms (Figure R18g,l).165

\section{Summary}

In conclusion, we investigated the actuation of patterned bilayers placed on a substrate. Due to the edge-activation of the bilayers, the observed deformed shapes differ from the classical ones obtained by homogeneous activation. We found that films can demonstrate several kinds of actuation behavior such as wrinkling, bending and folding that result in a variety of shapes. Moreover, it was demonstrated that one can introduce hinges into the folded structure by proper design of the bilayer's external shape through diffusion without having to use site-selective deposition of active polymers. Experimental observations led us to derive four empirical rules. We then demonstrated how these rules can be used to direct the folding of edgeactivated polymer bilayers through a concrete example - the design of a 3D pyramid. 


\section{Multi-Step Folding of Porous Films}

\section{Motivation}

In the previous section, the formation of a pyramid was demonstrated. A pyramid, however, has triangular sides. A question arises: is it possible to design a polymer bilayer film which, after folding, will form a figure with nontriangular sides, e.g. a cube? Cross-like structures have already been successfully used to create a cube ${ }^{17}$. Here, welding due to capillary forces is used to drive the process. Moreover, the building blocks, or the sides, of the structure are made of metal and are thus unable to bend. It was shown that this design gives the best results in terms of reproducibility, so we decided to adopt it for our system. However, in our case the sides would be made of a soft polymer which sports significantly lower mechanical stability and is thus susceptible to bending and folding. As it was already shown, swelling starts from the edges of the polymer bilayer, as this is the only location accessible for the solvent. If the radius of folding is significantly smaller than the size of the structure itself, tubes are formed from all sides. In this case, a square would stay a square but with its sides being tubes, i.e. rigid structures. As it was shown in the first section, subsequent folding of such squares leads to the formation of triangles. From the first sight, it seems pointless to use this multi-step approach for the design of cubes. Nevertheless, a cross-like structure made of squares differs from a single square. When multiple squares are joined together, some of their sides are no more the edges of the structure and are thus inaccessible for the solvent. As swelling starts from the edges of the polymer bilayer, a figure made by joined together squares should exhibit a different folding behavior than a single square. With all these considerations in mind, we found it worth investigating how such a structure would fold. Figure R24 represents the design (a) and the folding behavior ( $b-d)$ of a cross-like polymer bilayer. The structure was designed by joining six squares of the same size (marked with numbers on Figure R24a). As we needed the structure to have square-shaped sides after folding, we used squares with rounded sides in order to avoid distortions in the square 
form after formation of the tubes. If "normal" squares would have been used, the formation of tubes would have led to concave sides.

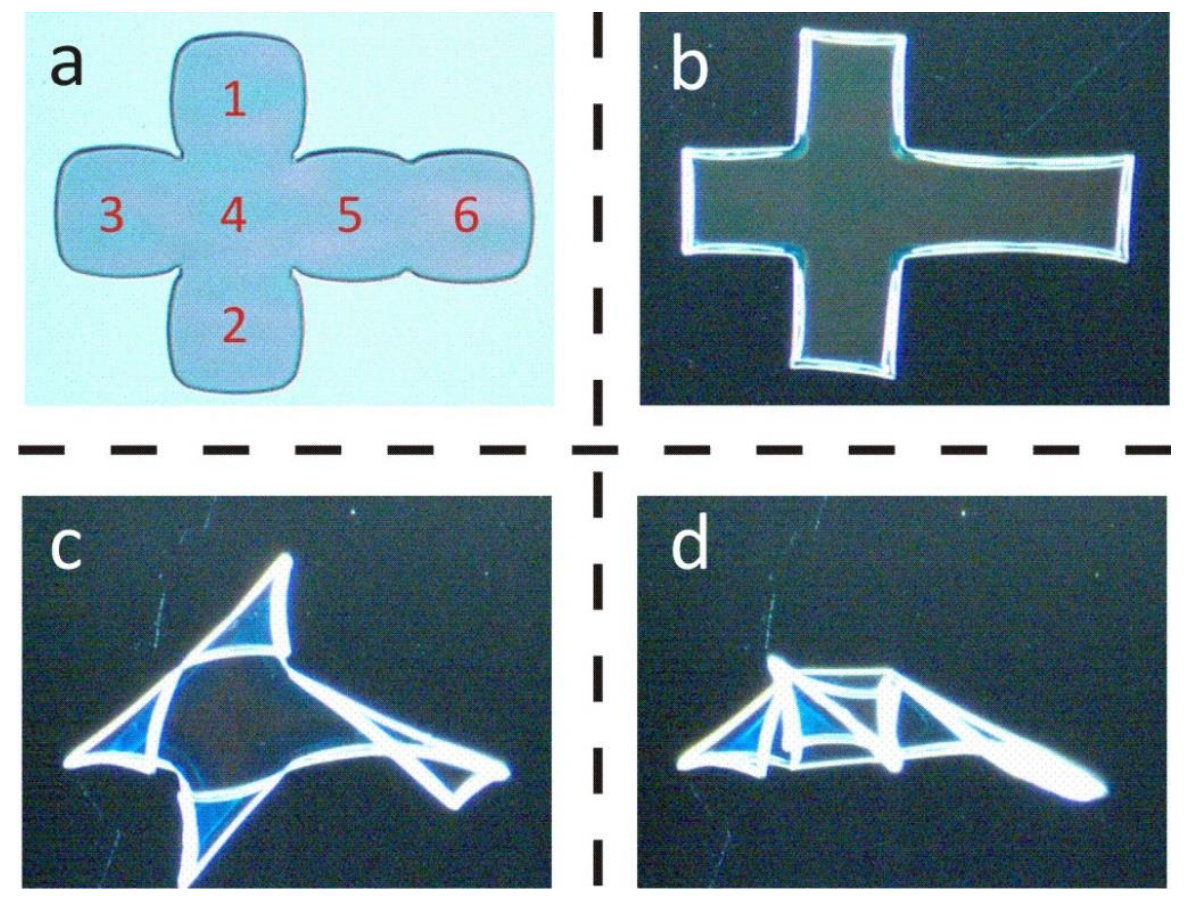

Figure R24. Folding of a cross-like polymer bilayer

The first step of the process went according to our expectations, namely, the edges of the structure formed tubes upon swelling. Parts 1,2 and 3 formed squares. Part 4 didn't swell, as it was inaccessible for the solvent. The sides of parts 5 and 6 , however, coalesced and formed a rectangle instead of two separate squares (Figure R24b). As the depth of the swelling front increased further, squares 1-3 folded along one of their diagonals into triangles. The rectangle formed from parts 5 and 6 also folded along one of its diagonals. As a result, the structure shown on Figure R24c was obtained. The last step of folding resulted in the structure, shown on Figure R24d. This structure, despite looking far from being a cube, allowed us to draw an important conclusion, namely, that hierarchical multi-step folding of square polymer bilayers would always tend to result (where possible) in the formation of triangles, whether one side of the square is connected to another structure or not. In order to create a cube or any other 3D structure with nontriangular sides, an effective way to stop folding along the lines connecting neighboring vertexes should be thought of. A possible solution would be the 
insertion of a rigid element inside the bilayer perpendicular to the direction of folding. How can this be done without the employment of expensive and timeconsuming methods like multi-step photolithography? The answer to this question is, albeit counter-intuitive, quite simple - one should design structures with pores. A pore would normally decrease the rigidity of a structure. A pore in our case is basically an edge inside the structure and can thus form tubes which, as we've already shown, exhibit much higher rigidity than a film. On the other hand, a pore exposes different parts of the active layer to the solvent and will strongly influence its swelling and, as a consequence, the folding behavior. Hence, we needed to investigate the influence of pores on the swelling and the folding behavior of polymer bilayers. We chose squares because of the simplicity of the form and the predictability of its hierarchical folding: a square is symmetrical and has only two diagonals. As a result, multi-step folding of a square leads to the formation of a triangle. Moreover, due to the symmetry of the figure, it doesn't matter along which diagonal folding will occur, as the outcome will always be the same. It is expected that the introduction of holes in the polymer film should strongly influence the swelling scenario, its folding behavior and thus shall change the shape of the resulting structure. Here, we investigate the influence of holes of different size and shapes on the folding behavior of polymer bilayers. We chose squares as a template form (further template squares) for our study, as there are only two possible ways for a hierarchical folding to occur, namely along either of the diagonals.

\section{Experimental part}

\section{Preparation and properties of the films.}

We prepared polymer films consisting of an active (lower) and passive (upper) layers as described earlier. For the active layer we used a random copolymer of $\mathrm{N}$-isopropylacrylamide (NIPAM, 92\% mol), acrylic acid (AA, 6\% mol) and 4-benzophenone acrylate (BA, 2\% mol) - P(NIPAM-AA). P(NIPAM AA) has a lower critical solution temperature (LCST) of around $28^{\circ} \mathrm{C}$ in water and more than $35^{\circ} \mathrm{C}$ in phosphate buffer with $\mathrm{pH}$ 7.4. In our study, however, 
we were not interested in the reversibility of the folding, so we used $1 \mathrm{mM}$ solution of sodium hydroxide $(\mathrm{NaOH})$ instead of water or buffer that increase the LCST to around $70^{\circ} \mathrm{C}$. Additionally, full folding of the bilayer occurred slowly (up to 1 hour) in buffer and even slower in water, while in $1 \mathrm{mM} \mathrm{NaOH}$ it took several minutes. The passive layer was a random copolymer of methylmethacrylate (MMA, 98\% mol) and 4-benzophenone acrylate (BPA, 2\% mol) - PMMA. The thicknesses of the resulting layers were $1200 \mathrm{~nm}$ for the $\mathrm{P}$ (NIPAM-AA) and $400 \mathrm{~nm}$ for the PMMA. These thicknesses led to the formation of tubes with a relatively small diameter (5-10 microns) compared to the size of the pores (20-200 microns) and the investigated patterned forms (500x500 microns. The patterned bilayer is underformed at $70^{\circ} \mathrm{C}$ and bends when the temperature decreases below $70^{\circ} \mathrm{C}$ due to swelling of the photocrosslinked P(NIPAM-AA) layer (Figure R25a).

\section{Results and discussion.}

\section{Folding of squares without holes}

First, we demonstrated a scenario for swelling and folding of square rectangles without holes (Figure R25b-d). Similar to our previous observations, swelling starts from the periphery of the films and the swelling front slowly moves towards the center of the film. Folding starts in the areas where the film is swollen, i.e. at the periphery, which leads to the formation of wrinkles/folds along each side of the square (Figure R25c). These wrinkles/tubes increase the rigidity/bending modulus of each side that allows further folding along the line connecting the vertexes where the bending modulus of the film is unchanged. As a result, triangles are formed (Figure R25d). There is no preferential bending direction during the second step, i.e. the film can bend along any of the diagonals. We also observed that the formed triangle "kniks" and starts to fold along the line connecting the center on the long side and the opposing vertex (Figure R25d). 


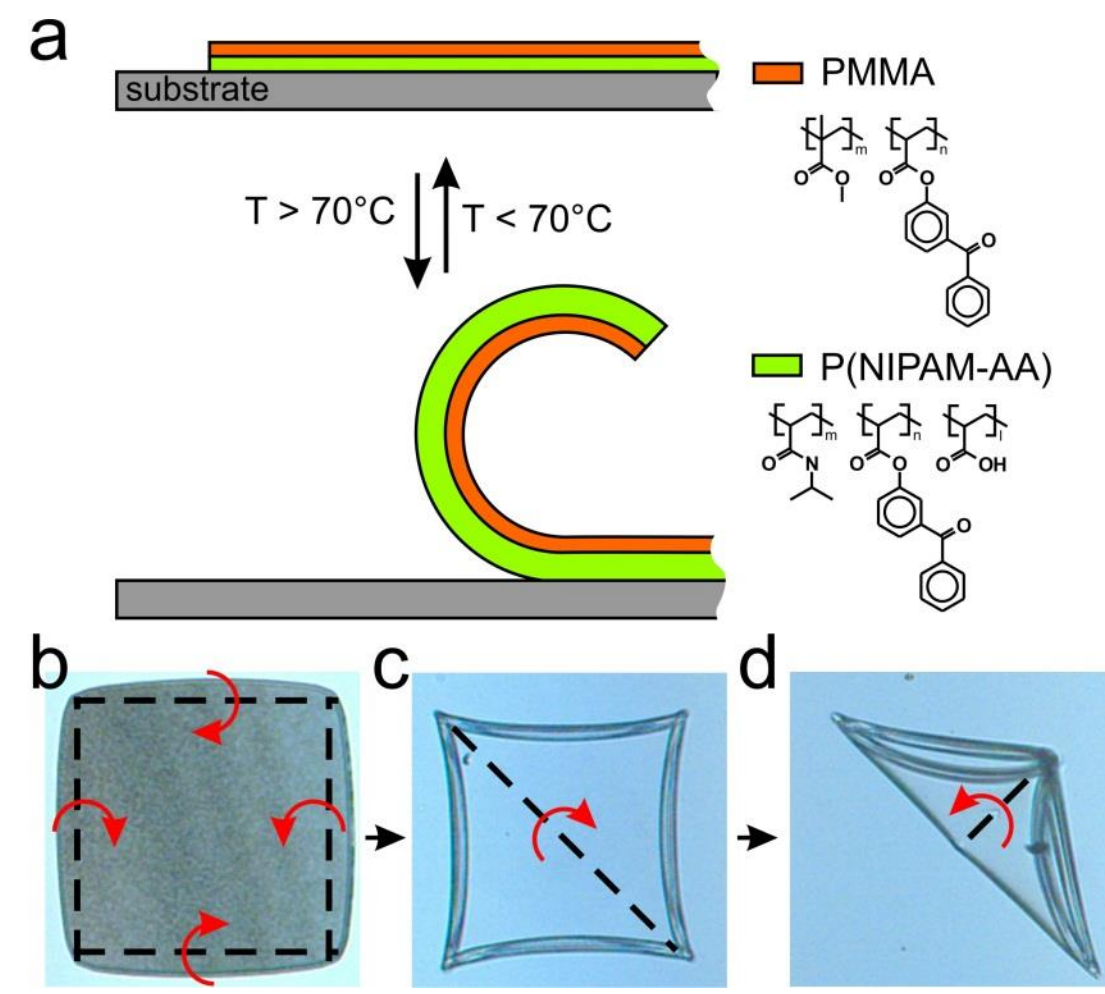

Figure R25. Folding of polymer bilayers. (a) - bending of a polymer bilayer consisting of hydrophobic PMMA and thermoresponsive P(NIPAM-AA); (b-d) microscopy images of the folding of a square film, (b) film before folding; (c) film after the first step of folding; (d) film after the second step of folding.

\section{Swelling of structures with holes}

It was previously shown that the substrate plays a crucial role in determining the folding behavior of polymer bilayers by limiting water diffusion to the lateral sides of the blend. However, a hole is essentially introducing additional edges inside the structure and will thus strongly influence the swelling pattern of the polymer films. In the light of this, the swelling behavior of porous structures had to be investigated in the first place. 

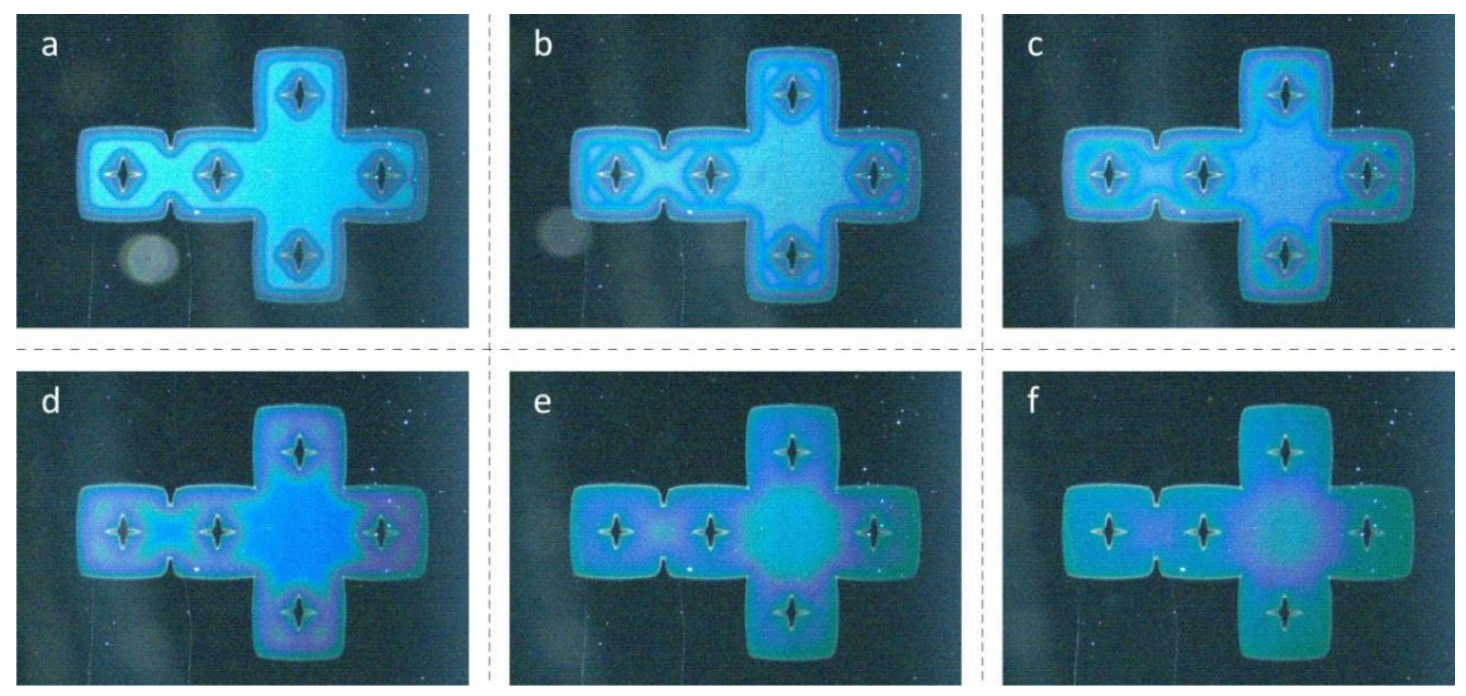

Figure R26. Swelling of porous structures. The swelling front is revealed through the changing colour of the polymer bilayer. Swelling starts at the outer edges of the structure, as well as at the pores.

An example of swelling of a complex porous structure is presented on Figure R26 (more information about the design of the structure is given later in the text). The investigation of swelling was performed in a qualitative manner by observing the color change of the films which reflects the change in the optical path length due to light interference (OPL). The OPL varies as a function of the film thickness and refractive index, which in turn depends on the swelling degree.

\section{Effect of a small hole position}

Second, we investigated the folding of squares with a different number of small holes located either in the center or near the corners of the squares. Swelling starts from both outer (square edges) and inner edges (holes) of the film. Folding follows the swelling scenario and starts from the outer and inner edges of the film. As a result, tubes along the square sides and small toroids inside the square are formed. The second step of folding is more complex than the folding of a simple square. First, similar to a simple square, the tubes along the square sides have higher rigidity, which leads to folding along the diagonals. What is different is that the toroids formed by the holes inside the square also sport increased rigidity. If the toroid is located on a possible folding line (diagonal), it hinders the folding. In the case of a single 
circular pore located in the center of the template square, bending along one of the diagonals still occurs with the bending line going round the toroid, as this is the direction of less resistance. Since both diagonals are equal, there is no preferential direction of folding.

Films with four circles/ellipsoids located close to the corners also fold and form a triangle (Figure R27a1). In this case, however, the folding line does not round the holes but goes through them, i.e. the toroids bend. Since an equal number of holes is positioned on each of the diagonals, there is no preferential direction of folding (Figure R27a2,a3). There is also no preferential folding direction if two holes are located on the same side of the square since the number of holes on the diagonals is the same (Figure R27b1b3). Template squares with two holes at the opposite corners (Figure R27c1) or three holes (Figure 2d1) break this symmetry. In this case, the diagonals are not equal (one hole on one of the diagonals and two holes on the other) and the film folds along the direction where the number of holes/toroids is smaller because the rigidity in this direction is smaller (Figure R27c2,d2).

symmetrical folding

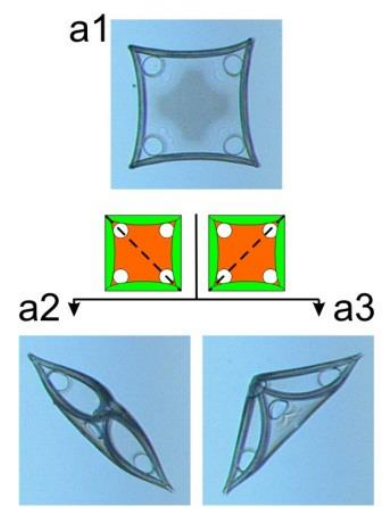

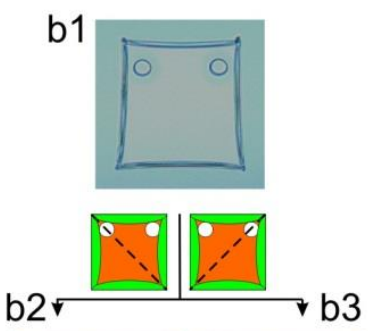

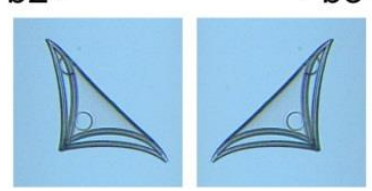

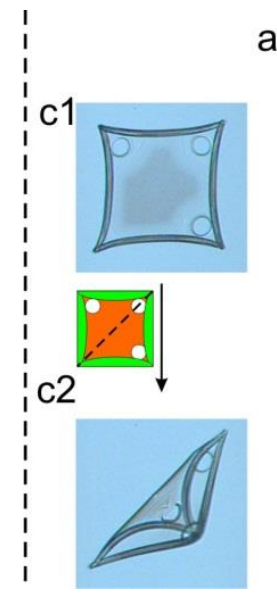

asymmetrical folding
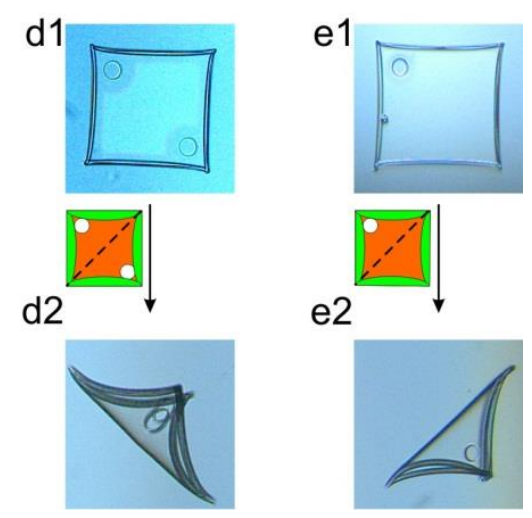

Figure R27. Different scenarios of folding of square bilayer with small circular holes: (a1-a3) holes are in all the corners - folding occurs along one of the diagonals; (b1-b3) holes are in three of the corners - folding always occurs along the diagonal with one pore only; (c1-c2) small two circular pores on different diagonals; (d1-d2) two circular pores on one diagonal - folding always occurs along the pore-free diagonal; (e1-e2) one circular pore in the corner - folding occurs along the diagonal without hole.

Similar behavior was observed for template squares with one small hole placed on one of the diagonals near the edge (Figure R27e1) - folding 
occurred only along the "pore-free" direction (Figure R27e2). Based on these observations one can conclude that (i) contrary to the apparent expectation that a hole shall make the film softer, it makes the film locally stiffer and (ii) there is a possibility to control the folding of the films by making and positioning of small holes in the film.

\section{Effect of shape and size}

Next, we investigated the effect of the size and shape of a hole located in the center of a square on the character of folding of a polymer film. Similar to the previous observations, swelling starts from the outer and inner edges of the film. Folding follows the swelling scenario and wrinkles/tubes along the inner and outer edges are formed in the first step of folding. The shape of the wrinkles/tubes inside depends on the shape of the hole. For example, square and cross-like holes form squares. Circles and ellipses form circular and elliptical toroids, respectively.

As it was already mentioned, a small circular pore in the middle of the template square is unable to completely hinder the diagonal folding of the square bilayer and the second step of folding goes round the toroid. As a result, a triangle is formed (Figure R28f1-f3,i1-i3). Similar effect should then be expected for all the cases where a small hole is positioned in the center of the template square in such a way, that after swelling it would form toroid/tube on the folding direction. Indeed, square and cross-like holes positioned in that manner change the line of bending so that it rounds the central pore. This is the case with square holes, where the square pore is turned to $45^{\circ}$ to the template square (Figure R28g1-g3,i1-i3). Surprisingly, the same behavior was observed if the pore was oriented the same way as the template square (Figure R28d1-f3). A closer look at the pore, however, reveals that the pore has an almost circular form, providing an explanation of the observed phenomenon: a small square pore forms a circle; subsequent folding then occurs as in the case of a circular pore. As expected, similar results were obtained with cross-like pores, as we specifically designed them to form squares after swelling of the active layer. 
We observed that in many cases the film underwent a third step of folding - further deformation of the triangles. The third step results in folding along the line connecting the center on the long side and the opposing vertex that is similar to Figure R26b-d or formation of more complex structures (Figure R28a3-i3). Increasing the size of the pores significantly changes the folding behavior of the polymer bilayers. Here, in contrast to the case of small pores, different pore shapes led to different folding tendencies. In general, all structures formed can be classified into three groups. The first group comprises simple diagonal folding (Figure R28f3,i3), which was discussed above. The second group comprises deformed diagonal-folded squares somewhat resembling a folded napkin (Figure R28a3,c3,d3,e3). The third group is represented by complexly folded structures as in Figure R28b3,h3.

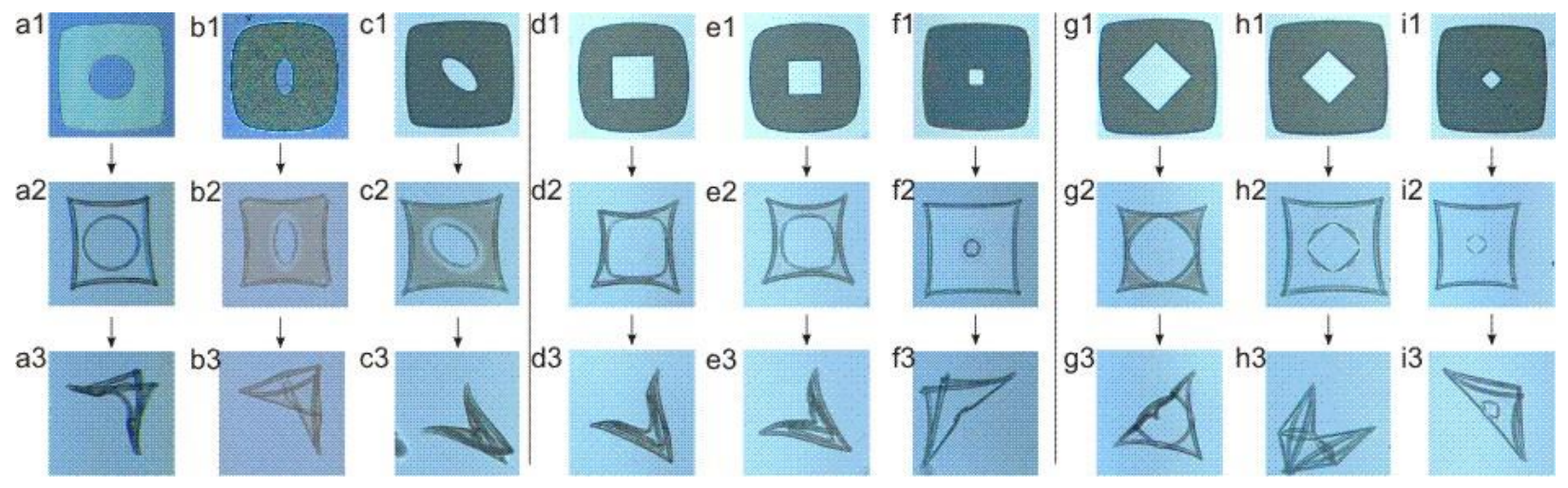

Figure R28. Folding of square bilayers with large holes of different shapes located in the center

\section{Mechanisms of folding}

Further, we investigated the process of formation of the second and third types of structures in detail (Figure R29). First, we considered folding of a square with a square hole which is not turned (the sides of the square and the hole are parallel, Figure R29a1-a3). Tubes/wrinkles are formed along the perimeter of the square and the hole after the first step of folding (Figure R29a1). Since the diagonals of the square coincide it folds along one of the diagonals and a triangle with a hole is formed (Figure R29a2). Next, the opposite sides move in the direction of each other and the film folds in half (Figure R29a3). Noteworthy, the whole process is very fast - it takes tenths of 
a second for the template square to fold, opposed to seconds in the first group. Release of stress, built up during the folding process, could be one of the possible explanations of the phenomenon. The folding sides of the square hole are rolling away from each other, thus pulling and stretching the polymer film in the areas of the pore-corners. This builds up stress in the material, which is released during the folding process, greatly increasing its speed. Another peculiar thing about the process is that one of the sides of the final structure is folded in the wrong direction, i.e. the active layer forms the inner curvature of the bend, while the passive forms the outer. This is not only contrary to everything that has previously been observed, but also seems illogical at first glance, as it is the active layer that increases in volume and not the passive. Based on that we argue the structures are not fully symmetrical and one side dominates over the other. This also provides an explanation of the "knicks", observed in the case of pore-free template squares (see Figure R24). Here, one side dominates but the difference is too small compared to the overall area of the polymer film. In the case of square pores, however, the asymmetry is larger due to the smaller area of the polymer film, which makes it possible for the third step of folding to proceed further. Still, the third folding step doesn't succeed fully to the end - the structures are half-opened, in contrast to the second step, where almost fully closed triangles are formed. This can be due to the fact that in the third folding the "wrongly" bent side prevents the structure from folding to the full extent.

Next, we investigated the folding of a square with one large, circle-like hole (Figure R28a1-a3). Tubes along each side of the square and a toroid in the hole are formed after the first step of folding (Figure R29b1). The toroid has higher rigidity than the bilayer film and hinders the bending along the diagonals of the square. Therefore, the square bends along the diagonal with relatively large curvature radius, which exceeds the radius of curvature in the first step of folding. Similar to previous examples, further swelling leads to folding in half (Figure R29b3-b4).

Further, we investigated the folding of a square with a square hole turned by $45^{\circ}$ (Figure R29c1,d1). 


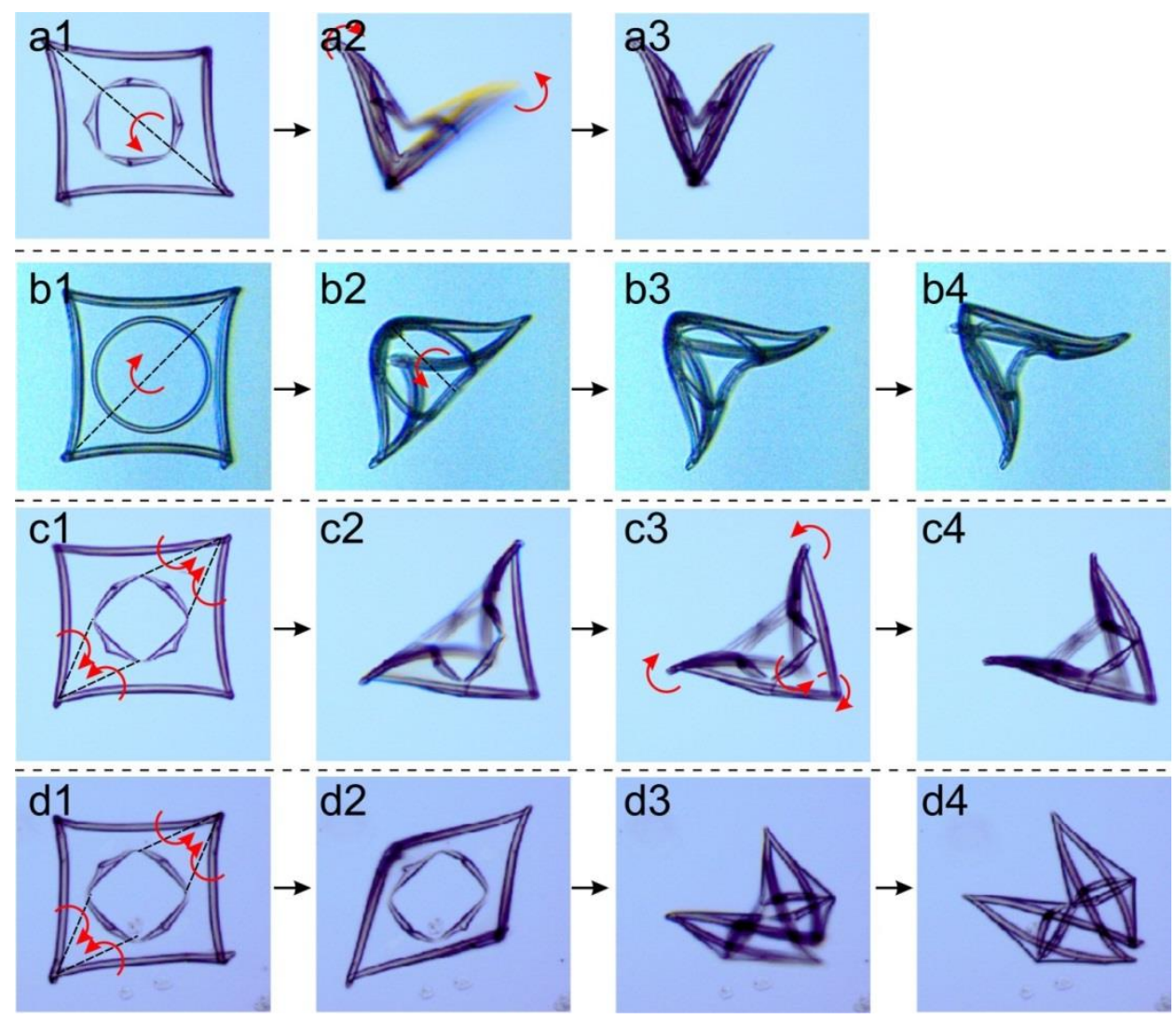

Figure R29. Microscopy snapshots on different stages of folding of square bilayer with holes of different shape

The first step of folding proceeds similarly to previous experiments and leads to the formation of tubes along the perimeter of the square and the hole. Here, folding along the diagonals is not favorable as it would require the tubes to be bent. Instead, the second step of folding occurs along the lines, connecting the vertexes of the template square with the vertexes of the square pore as shown on (Figure R29c1-c3,d1-d2, Figure R30b1-b3). Further folding proceeds in a manner that two opposing vertexes bend towards each other (Figure R29c3). At the end of the process, the square pore may preserve its form (Figure R29c4, Figure R30b1,c1). Another possibility is that the square hole collapses (Figure R29d4, Figure R30b2,c2,d1,e1). Very rarely, we observed that two side of the holes bend, leading to a structure presented on Figure R30b3,c3,d2,e2. We only observed this type of behavior in less than 1\% of the folding events. 


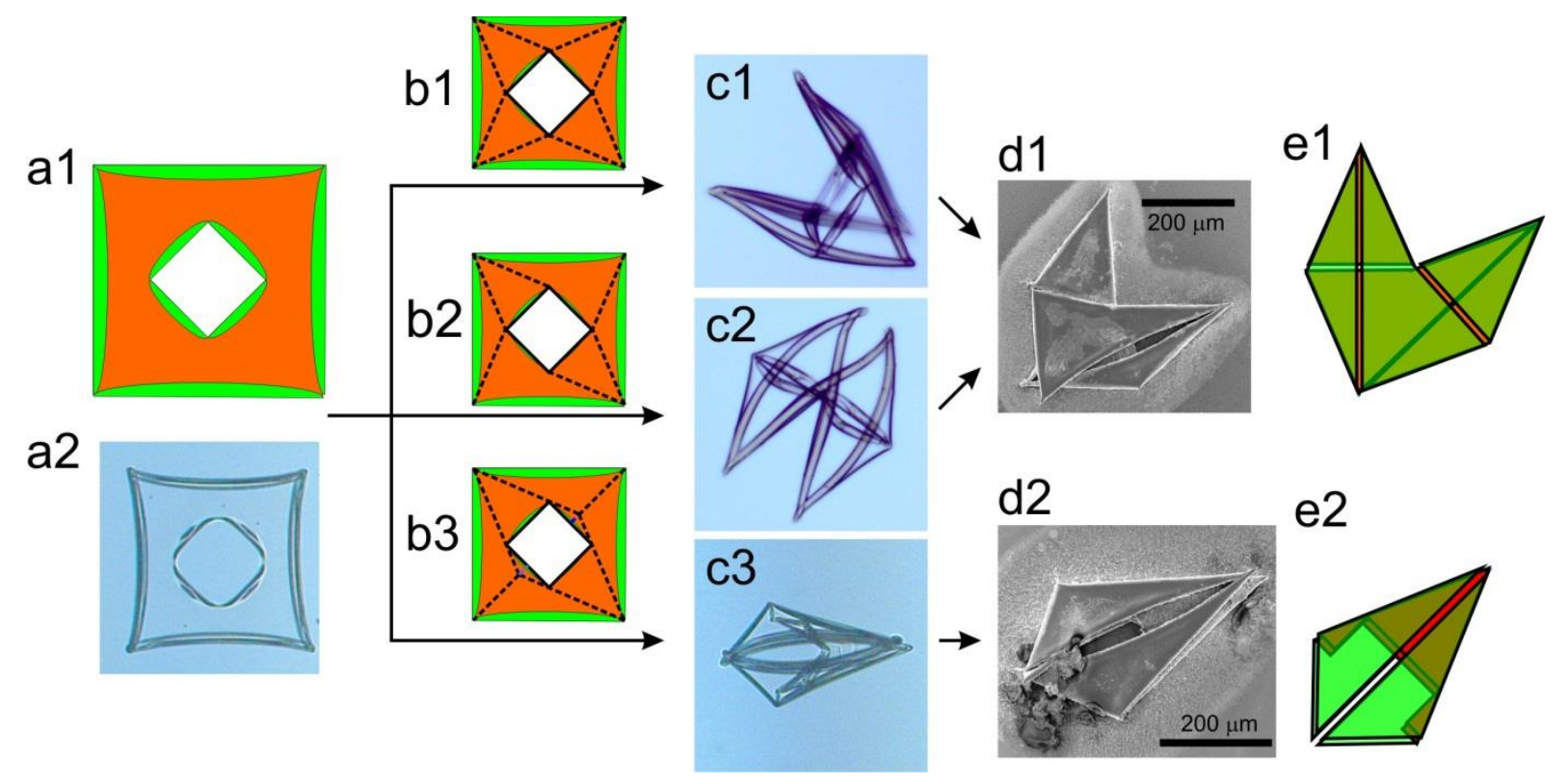

Figure R30. Folding scenarios of a square with a cross-like hole turned by $45^{\circ}$

Going back to the motivation of this part of the work, one thing we wanted to achieve was to obtain a cube by hierarchical multi-step folding of a cross-like polymer bilayer. As it was already shown, a pore-free template failed to fulfill the task as folding of the to-be-sides lead to the formation of triangles, i.e. two-step folding occurred. So far, all of the suggested designs failed to hinder the two- and three-step folding of the template squares, which proceeded either along the diagonals or in a more sophisticated manner, as in the case with square pores. Based on these results, one could argue that introducing pores to the template cross-like structure would also fail to direct the folding in the desired direction, i.e. to the formation of a cubical. Nevertheless, we considered it worth investigating. For this purpose, structures shown on Figure R31 a1 and b1 were designed.

Similarly to the previous pattern (see Figure R24), these were obtained by joining squares with pores together. One of the squares was left pore-free, because it was already observed that it doesn't fold. The architecture also exhibited larger distance between squares 5 and 6 . This was done in order to avoid the forming tubes from coalescing, as was in the case with the previous design. Typical folding behavior of the polymer bilayer is shown on Figure R31. 

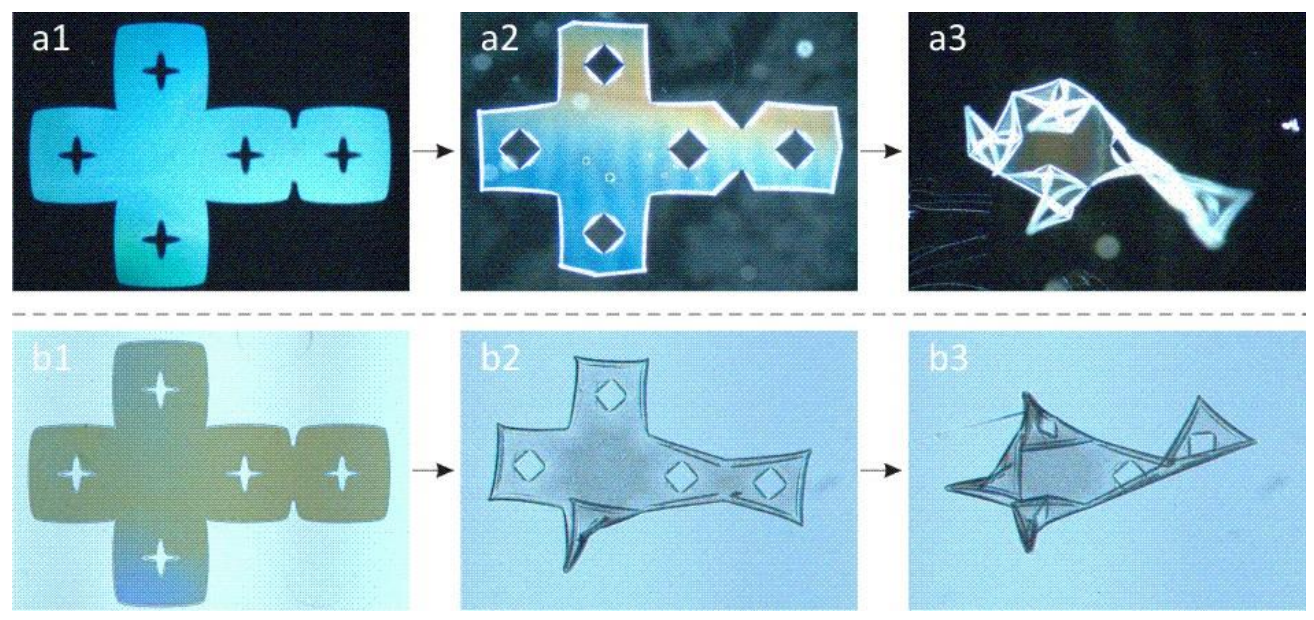

Figure R31. Folding of a cross-like polymer bilayer with square holes

First, tubes are formed (Figure R31a2 and b2). Subsequently, squares 1,2 and 4 undergo second-step folding and form structures, identical to the ones formed by the template squares (compare with fig R29). Part 5 and 6 , albeit not forming a single rectangle due to coalescence of their sides, showed more random behavior with folding occurring along any possible line that didn't cross a tube.

\section{Summary}

We investigated the influence of pores on the folding behavior of square-shaped polymer bilayers. Furthermore, we showed that pores of the right form and dimensions can be actively used to change the folding of a polymer bilayer by hindering the folding in certain (chosen) directions, thus allowing for the design of novel 3D structures. We also showed a novel type of folding of such systems, namely reverse folding, which further increases the arsenal of available structures. 


\section{Summary and Conclusions}

\section{Chapter}

- Summary and Conclusions

- Outlook

- References
This thesis discusses the preparation of self-folding polymer bilayers and the investigation of their swelling and folding behavior. This section helps to understand the connection between the different chapters, gives a condensed insight on the results and reviews the most important considerations and analysis. In particular, the influence of factors like folding radii and the presence of holes on the folding nature of simple (rectangle) and complex (star-like) shapes was thoroughly investigated and the acquired knowledge was then used to design various $3 \mathrm{D}$ constructs.

One of the main goals of this work was to explore the possibilities and to find different from the already reported methods for assembling of polymer bilayers into 3D origami structures. We found that the existing at the time 
this work started information on the folding behavior of bilayer systems was not complete and explicit enough for our purpose. As a consequence, we had to start our research on basic and thus easy-to-understand shapes like rectangles.

It was shown that swelling of a bilayer system starts from the periphery due to faster diffusion of water and propagates inside.

Furthermore, folding follows the swelling scenario and also starts from the periphery. It then proceeds in one step, if the folding curvature is comparable with the size of the object and leads to the formation of rounded objects such as tubes and capsules. If the bending radius is much smaller than the lateral dimensions of the structure, multistep folding is observed, yielding objects with sharp edges.

Rectangle films with small folding diameters $(\mathrm{C}<<\mathrm{L})$ undergo long-side rolling at high aspect ratios. At low aspect ratios, diagonal and mixed types of rolling were observed. For large folding diameters $(\mathrm{C} \approx \mathrm{L})$, long-side rolling prevails at high aspect ratios; all-side rolling is typical for low aspect ratios. Multi-step folding occurs when the radius is small and is directed by the $2 \mathrm{D}$ shape of the film. Based on the analysis of various folding scenarios, empirical rules for the process were derived:

1) "Bilayer polymer films placed on a substrate start to fold from their periphery and the number of formed wrinkles/tubes decreases until the angle between adjacent wrinkles/tubes approaches 130\%;

2) "After the wrinkles along the perimeter of the film form tubes, further folding proceeds along the lines connecting the vertexes of the folded film";

3) "The folding goes along the lines which are closer to the periphery of the films";

4) „Folding of the rays may result in blocking of the neighboring rays if the angle between the base of the folded ray and the shoulders of the neighboring rays is close to $180^{\circ}$ ".

By applying these rules we designed experiments to obtain different 2D and $3 \mathrm{D}$ constructs, e.g. pyramids. The formation of a cube in a similar way, 
however, proved to be futile, as its square sides undergo folding to form triangles. To overcome the problem, we suggested that holes were introduced in the polymer bilayers. The main reason is that a hole will form tubes that, as we already knew, can act as rigid elements, effectively blocking the folding in the perpendicular to it direction. Despite the correctness of our reasoning, we failed to produce a cube by hierarchical folding of polymer bilayers. However, other sophisticated 3D objects were obtained, further increasing the arsenal of available structures, as well as giving an in-depth insight on the folding process. 


\section{Outlook}

Since the second industrial revolution $19^{\text {th }}$ century, humanity has experienced truly rapid incorporation of ever newer technologies in all spheres of life. Some of these innovations, alongside with their obvious benefits for the growth and prosperity of the society, can pose certain risks and cause complications. As a result, with ever increasing development and application of new scientific ideas, nowadays humanity faces several globalscale problems. Three major areas of concern are healthcare, energy and environment, representing the inner will of all the people to live long and healthy in a clean world, with no lack in resources and mobility.

There is an ever increasing trend among scientists and engineers to use nature as a source of inspiration, successfully copying and even improving different structures, properties and mechanism from living organisms and applying them to solve problems. "Bioispired" and "biomimetics" are often found in the description of numerous recent publications, especially in medicine and bioengineering. The self-folding polymer bilayers are no exception, as they mimic the mechanism of actuation in plants.

In the last decades, regenerative therapy has become an increasingly demanded branch of medicine. As an important part of it, tissue engineering has also attracted significant interest from the scientific community. Tissue engineering is associated with applications that repair or replace portions of or whole tissues, for example bone, cartilage, blood vessels. To create a tissue, cells are implanted into an artificial structure capable of supporting threedimensional tissue formation - the so called scaffold. A biodegradable scaffold is readily broken down by enzymes and can thus be dissolved after it has fulfilled its function. The properties of the scaffold play a crucial role in the development of the tissue. Despite the rapid advance in this field, there is a constant need for novel materials and cell-loading methods for scaffolds. In 
most cases, the whole scaffold is manufactured at first, and then cells are incorporated inside it by means of different techniques. As a result of slow migration of cells through a porous scaffold, cells may be inhomogeneously loaded. The inhomogeneity of loading can be solved using the self-folding structures.

The self-folding microstructures presented in this work represent an excellent potential approach for the formation of 3D structures such as scaffolds, possessing numerous advantages. First, by means of chemical modification or simply by the proper choice of monomers, self-folding structures can easily be made biocompatible and biodegradable. Next, the self-folding approach gives the possibility to first prepare structural elements for the scaffold, then load them with cells in a desired manner and then assemble the elements in a 3D aggregate, theoretically allowing for a far better control over the loading parameters. Porosity is an important factor for the survival of cells inside the scaffold.

Another perspective direction for the further development and application of this work is the design of biocompatible self-propelled microjets. Microjets based on inorganic materials have been in the center of attention of several research groups, as they possess a number of attractive properties that make them ideal candidates for cell-manipulation tools. In one approach, for example, freely floating cells are pushed by a microjet. The speed of movement is determined the $\mathrm{H}_{2} \mathrm{O}_{2}$ concentration and the diameter of the tube and the direction of swimming can be controlled by a magnet. The concentration of $\mathrm{H}_{2} \mathrm{O}_{2}$ in the human body is, however, quite low and for the successful application of this approach in vivo other means of producing gas should be found. Another way to grant the ability to move the microtubes is to encapsulate cells that are capable of directed movement, e.g. sperm cells. Here, the tube is propelled by the cell and incorporation of magnetic layers in it would allow to easily control its direction. This cell-laden tube can ultimately be used as a microcarrier for drugs, particles or even other cells.

This work has provided the basic fundamentals of folding continuous as well as porous bilayers. Both theoretical considerations and experimental 
results have shown the possibility to create sophisticated shapes, suitable for various applications in medicine and biotechnology. This work can thus be considered a step towards the design of innovative materials. 


\section{References}

1. Gracias, D. H.; Tien, J.; Breen, T. L.; Hsu, C.; Whitesides, G. M., Forming electrical networks in three dimensions by self-assembly. Science 2000, 289, (5482), 1170-1172.

2. Leong, T.; Gu, Z. Y.; Koh, T.; Gracias, D. H., Spatially controlled chemistry using remotely guided nanoliter scale containers. Journal of the American Chemical Society 2006, 128, (35), 11336-11337.

3. Guo, X. Y.; Li, H.; Ahn, B. Y.; Duoss, E. B.; Hsia, K. J.; Lewis, J. A.; Nuzzo, R. G., Two- and three-dimensional folding of thin film single-crystalline silicon for photovoltaic power applications. Proceedings of the National Academy of Sciences of the United States of America 2009, 106, (48), 20149-20154.

4. Kwon, G. H.; Choi, Y. Y.; Park, J. Y.; Woo, D. H.; Lee, K. B.; Kim, J. H.; Lee, S.-H., Electrically-driven hydrogel actuators in microfluidic channels: fabrication, characterization, and biological application. Lab on a Chip 2010, 10, (12), 1604-1610.

5. Zakharchenko, S.; Puretskiy, N.; Stoychev, G.; Stamm, M.; Ionov, L., Temperature controlled encapsulation and release using partially biodegradable thermo-magneto-sensitive self-rolling tubes. Soft Matter 2010, 6, (12), 2633-2636.

6. Zakharchenko, S.; Sperling, E.; Ionov, L., Fully biodegradable self-rolled polymer tubes: a candidate for tissue engineering scaffolds. Biomacromolecules 2011, $12,(6), 2211-2215$.

7. Leong, T. G.; Randall, C. L.; Benson, B. R.; Zarafshar, A. M.; Gracias, D. H., Self-loading lithographically structured microcontainers: 3D patterned, mobile microwells. Lab on a Chip 2008, 8, (10), 1621-1624.

8. Jamal, M.; Bassik, N.; Cho, J. H.; Randall, C. L.; Gracias, D. H., Directed growth of fibroblasts into three dimensional micropatterned geometries via selfassembling scaffolds. Biomaterials 2010, 31, (7), 1683-90. 
9. Guan, J. J.; He, H. Y.; Lee, L. J.; Hansford, D. J., Fabrication of particulate reservoir-containing, capsulelike, and self-folding polymer microstructures for drug delivery. Small 2007, 3, (3), 412-418.

10. Shim, T. S.; Kim, S.-H.; Heo, C.-J.; Jeon, H. C.; Yang, S.-M., Controlled Origami Folding of Hydrogel Bilayers with Sustained Reversibility for Robust Microcarriers. Angewandte Chemie-International Edition 2012, 51, (6), 1420-1423.

11. Iriso Seimetsu Co., L. http://www.tanomi.com/limited/html/00048.html

12. Ionov, L., Soft microorigami: self-folding polymer films. Soft Matter 2011, 7, 6786-6791

13. Randall, C. L.; Kalinin, Y. V.; Jamal, M.; Manohar, T.; Gracias, D. H., Threedimensional microwell arrays for cell culture. Lab on a Chip 2011, 11, (1), 127-131.

14. Jager, E. W. H.; Inganas, O.; Lundstrom, I., Microrobots for micrometer-size objects in aqueous media: Potential tools for single-cell manipulation. Science 2000, 288, (5475), 2335-2338.

15. Leong, T. G.; Randall, C. L.; Benson, B. R.; Bassik, N.; Stern, G. M.; Gracias, D. H., Tetherless thermobiochemically actuated microgrippers. Proceedings of the National Academy of Sciences of the United States of America 2009, 106, (3), 703708.

16. Huang, G.; Mei, Y.; Thurmer, D. J.; Coric, E.; Schmidt, O. G., Rolled-up transparent microtubes as two-dimensionally confined culture scaffolds of individual yeast cells. Lab Chip 2009, 9, (2), 263-8.

17. Azam, A.; Leong, T. G.; Zarafshar, A. M.; Gracias, D. H., Compactness Determines the Success of Cube and Octahedron Self-Assembly. PLoS ONE 2009, 4, (2), e4451.

18. Behl, M.; Razzaq, M. Y.; Lendlein, A., Multifunctional Shape-Memory Polymers. Advanced Materials 2010, 22, (31), 3388-3410.

19. Leong, T. G.; Benson, B. R.; Call, E. K.; Gracias, D. H., Thin film stress driven self-folding of microstructured containers. Small 2008, 4, (10), 1605-9. 
20. Liu, Y.; Boyles, J. K.; Genzer, J.; Dickey, M. D., Self-folding of polymer sheets using local light absorption. Soft Matter 2012, 8, 1764-1769.

21. Ohm, C.; Brehmer, M.; Zentel, R., Liquid Crystalline Elastomers as Actuators and Sensors. Advanced Materials 2010, 22, (31), 3366-3387.

22. Wu, C.; Wang, X., Globule-to-Coil Transition of a Single Homopolymer Chain in Solution. Physical Review Letters 1998, 80, (18), 4092-4094.

23. Guan, Y.; Zhang, Y., PNIPAM microgels for biomedical applications: from dispersed particles to 3D assemblies. Soft Matter 2011, 7, (14), 6375-6384.

24. Kumar, D.; Sharma, R. C., Advances in conductive polymers. European Polymer Journal 1998, 34, (8), 1053-1060.

25. Leong, T. G.; Zarafshar, A. M.; Gracias, D. H., Three-dimensional fabrication at small size scales. Small 2010, 6, (7), 792-806.

26. Hawkes, E.; An, B.; Benbernou, N. M.; Tanaka, H.; Kim, S.; Demaine, E. D.; Rus, D.; Wood, R. J., Programmable matter by folding. Proceedings of the National Academy of Sciences.

27. Smela, E.; Inganas, O.; Lundstrom, I., CONTROLLED FOLDING OF MICROMETER-SIZE STRUCTURES. Science 1995, 268, (5218), 1735-1738.

28. Solovev, A. A.; Sanchez, S.; Pumera, M.; Mei, Y. F.; Schmidt, O. G., Magnetic Control of Tubular Catalytic Microbots for the Transport, Assembly, and Delivery of Micro-objects. Advanced Functional Materials 2010, 20, (15), 2430-2435.

29. Huang, G. S.; Mei, Y. F.; Thurmer, D. J.; Coric, E.; Schmidt, O. G., Rolled-up transparent microtubes as two-dimensionally confined culture scaffolds of individual yeast cells. Lab on a Chip 2009, 9, (2), 263-268.

30. Smith, E. J.; Liu, Z.; Mei, Y. F.; Schmidt, O. G., System investigation of a rolled-up metamaterial optical hyperlens structure. Applied Physics Letters 2009, $95,(8),-$. 
31. Luchnikov, V.; lonov, L.; Stamm, M., Self-rolled polymer tubes: novel tools for microfluidics, microbiology, and drug-delivery systems. Macromol Rapid Commun 2011, 32, (24), 1943-52.

32. Luchnikov, V.; Stamm, M., Self-rolled polymer microtubes with engineered hidden walls. Physica E: Low-dimensional Systems and Nanostructures 2007, 37, (12), $236-240$.

33. Bof Bufon, C. C. s.; Cojal González, J. D.; Thurmer, D. J.; Grimm, D.; Bauer, M.; Schmidt, O. G., Self-Assembled Ultra-Compact Energy Storage Elements Based on Hybrid Nanomembranes. Nano Letters 2010, 10, (7), 2506-2510.

34. Schwaiger, S.; Broll, M.; Krohn, A.; Stemmann, A.; Heyn, C.; Stark, Y.; Stickler, D.; Heitmann, D.; Mendach, S., Rolled-Up Three-Dimensional Metamaterials with a Tunable Plasma Frequency in the Visible Regime. Physical Review Letters 2009, 102, (16), - .

35. Terfort, A.; Bowden, N.; Whitesides, G. M., Three-dimensional self-assembly of millimetre-scale components. Nature 1997, 386, (6621), 162-164.

36. Whitesides, G. M.; Grzybowski, B., Self-Assembly at All Scales. Science 2002, 295 , (5564), 2418-2421.

37. Cai, S.; Chen, D.; Suo, Z.; Hayward, R. C., Creasing instability of elastomer films. Soft Matter 2012, 8, (5), 1301-1304.

38. Singamaneni, S.; Tsukruk, V. V., Buckling instabilities in periodic composite polymeric materials. Soft Matter 2010, 6, (22), 5681-5692.

39. Yang, S.; Khare, K.; Lin, P. C., Harnessing Surface Wrinkle Patterns in Soft Matter. Advanced Functional Materials 2010, 20, (16), 2550-2564.

40. Hendricks, T. R.; Wang, W.; Lee, I., Buckling in nanomechanical films. Soft Matter 2010, 6, (16), 3701-3706.

41. Chen, X.; Yin, J., Buckling patterns of thin films on curved compliant substrates with applications to morphogenesis and three-dimensional microfabrication. Soft Matter 2010, 6, (22), 5667-5680. 
42. Schweikart, A.; Pazos-Perez, N.; Alvarez-Puebla, R. A.; Fery, A., Controlling inter-nanoparticle coupling by wrinkle-assisted assembly. Soft Matter 2011, 7, (9), 4093-4100.

43. Schweikart, A.; Fery, A., Controlled wrinkling as a novel method for the fabrication of patterned surfaces. Microchimica Acta 2009, 165, (3-4), 249-263.

44. Genzer, J.; Groenewold, J., Soft matter with hard skin: From skin wrinkles to templating and material characterization. Soft Matter 2006, 2, (4), 310-323.

45. Chan, E. P.; Smith, E. J.; Hayward, R. C.; Crosby, A. J., Surface wrinkles for smart adhesion. Advanced Materials 2008, 20, (4), 711-+.

46. Chan, E. P.; Crosby, A. J., Spontaneous formation of stable aligned wrinkling patterns. Soft Matter 2006, 2, (4), 324-328.

47. Guvendiren, M.; Burdick, J. A.; Yang, S., Solvent induced transition from wrinkles to creases in thin film gels with depth-wise crosslinking gradients. Soft Matter 2010, 6, (22), 5795-5801.

48. Guvendiren, M.; Yang, S.; Burdick, J. A., Swelling-Induced Surface Patterns in Hydrogels with Gradient Crosslinking Density. Advanced Functional Materials 2009, 19, (19), 3038-3045.

49. Kim, H. S.; Crosby, A. J., Solvent-Responsive Surface via Wrinkling Instability. Advanced Materials 2011, 23, (36), 4188-+.

50. Chung, J. Y.; Youngblood, J. P.; Stafford, C. M., Anisotropic wetting on tunable micro-wrinkled surfaces. Soft Matter 2007, 3, (9), 1163-1169.

51. Zhao, Y.; Huang, W. M.; Fu, Y. Q., Formation of micro/nano-scale wrinkling patterns atop shape memory polymers. Journal of Micromechanics and Microengineering 2011, 21, (6).

52. Bowden, N.; Brittain, S.; Evans, A. G.; Hutchinson, J. W.; Whitesides, G. M., Spontaneous formation of ordered structures in thin films of metals supported on an elastomeric polymer. Nature 1998, 393, (6681), 146-149. 
53. Ohzono, T.; Matsushita, S. I.; Shimomura, M., Coupling of wrinkle patterns to microsphere-array lithographic patterns. Soft Matter 2005, 1, (3), 227-230.

54. Efimenko, K.; Rackaitis, M.; Manias, E.; Vaziri, A.; Mahadevan, L.; Genzer, J., Nested self-similar wrinkling patterns in skins. Nature Materials 2005, 4, (4), 293-297.

55. Yoo, P. J.; Lee, H. H., Morphological diagram for metal/polymer bilayer wrinkling: Influence of thermomechanical properties of polymer layer. Macromolecules 2005, 38, (7), 2820-2831.

56. Vella, D.; Adda-Bedia, M.; Cerda, E., Capillary wrinkling of elastic membranes. Soft Matter 2010, 6, (22), 5778-5782.

57. Cendula, P.; Kiravittaya, S.; Mei, Y. F.; Deneke, C.; Schmidt, O. G., Bending and wrinkling as competing relaxation pathways for strained free-hanging films. Physical Review B 2009, 79, (8).

58. Schweikart, A.; Horn, A.; Boker, A.; Fery, A., Controlled Wrinkling as a Novel Method for the Fabrication of Patterned Surfaces. Complex Macromolecular Systems I 2010, 227, 75-99.

59. Huang, J.; Juszkiewicz, M.; de Jeu, W. H.; Cerda, E.; Emrick, T.; Menon, N.; Russell, T. P., Capillary wrinkling of floating thin polymer films. Science 2007, 317, (5838), 650-653.

60. Singamaneni, S.; McConney, M. E.; Tsukruk, V. V., Spontaneous Self-Folding in Confined Ultrathin Polymer Gels. Advanced Materials 2010, 22, (11), 1263-1268.

61. Horn, A.; Schoberth, H. G.; Hiltl, S.; Chiche, A.; Wang, Q.; Schweikart, A.; Fery, A.; Boker, A., Nanostructured wrinkled surfaces for templating bionanoparticles-controlling and quantifying the degree of order. Faraday Discussions 2009, 143, 143-150.

62. Klein, Y.; Efrati, E.; Sharon, E., Shaping of Elastic Sheets by Prescription of Non-Euclidean Metrics. Science 2007, 315, (5815), 1116-1120. 
63. Zhou, F.; Biesheuvel, P. M.; Chol, E. Y.; Shu, W.; Poetes, R.; Steiner, U.; Huck, W. T. S., Polyelectrolyte brush amplified electroactuation of microcantilevers. Nano Letters 2008, 8, (2), 725-730.

64. Timoshenko, S., Analysis of bi-metal thermostats. Journal of the Optical Society of America and Review of Scientific Instruments 1925, 11, (3), 233-255.

65. Mansfield, E. H., Bending, buckling and curling of a heated thin plate. Proc. R. Soc. A 1962, 268, (1334), 316-237.

66. Mansfield, E. H., Bending buckling and curling of a heated elliptical plate. Proc. R. Soc. A 1965, 288, (1414), 396-417.

67. Freund, L. B., Substrate curvature due to thin film mismatch strain in the nonlinear deformation range. J Mech Phys Solids 2000, 48, (6-7), 1159-1174.

68. Alben, S.; Balakrisnan, B.; Smela, E., Edge Effects Determine the Direction of Bilayer Bending. Nano Letters 2011, 11, (6), 2280-2285.

69. Chun, I. S.; Challa, A.; Derickson, B.; Hsia, K. J.; Li, X., Geometry Effect on the Strain-Induced Self-Rolling of Semiconductor Membranes. Nano Letters 2010, 10, (10), 3927-3932.

70. Cendula, P.; Kiravittaya, S.; Monch, I.; Schumann, J.; Schmidt, O. G., Directional Roll-up of Nanomembranes Mediated by Wrinkling. Nano Letters 2011, $11,(1), 236-240$.

71. Kim, J.; Hanna, J. A.; Hayward, R. C.; Santangelo, C. D., Thermally responsive rolling of thin gel strips with discrete variations in swelling. Soft Matter $2012,8,(8), 2375-2381$.

72. Serak, S.; Tabiryan, N.; Vergara, R.; White, T. J.; Vaia, R. A.; Bunning, T. J., Liquid crystalline polymer cantilever oscillators fueled by light. Soft Matter 2010, 6, (4), 779-783.

73. Bassik, N.; Brafman, A.; Zarafshar, A. M.; Jamal, M.; Luvsanjav, D.; Selaru, F. M.; Gracias, D. H., Enzymatically Triggered Actuation of Miniaturized Tools. Journal of the American Chemical Society 2010, 132, (46), 16314-16317. 
74. Gultepe, E.; Randhawa, J. S.; Kadam, S.; Yamanaka, S.; Selaru, F. M.; Shin, E. J.; Kalloo, A. N.; Gracias, D. H., Biopsy with Thermally-Responsive Untethered Microtools. Advanced Materials 2013, 25, (4), 514-519.

75. Fernandes, R.; Gracias, D. H., Toward a miniaturized mechanical surgeon. Materials Today 2009, 12, (10), 14-20.

76. Laflin, K. E.; Morris, C. J.; Bassik, N.; Jamal, M.; Gracias, D. H., Tetherless Microgrippers With Transponder Tags. Microelectromechanical Systems, Journal of $2011,20,(2), 505-511$.

77. A Long-Range Surface Plasmon Resonance /Probe/ Silver Nanoparticle (LRSPR-P-NP) Nano-antenna Configuration for Surface-Enhanced Raman Scattering. The Journal of Physical Chemistry Letters 2012, 120912152516003.

78. Wireless system for biological signal recording with Gallium Arsenide High Electron Mobility Transistors as sensing elements. Microelectronic Engineering 2013.

79. Fernandes, R.; Gracias, D. H., Self-folding polymeric containers for encapsulation and delivery of drugs. Advanced Drug Delivery Reviews 2012, 64, (14), 1579-1589.

80. Fontaine, C.; Gailliard, J. P.; Magli, S.; Million, A.; Piaguet, J., Relaxation of stresses in CdTe layers grown by molecular beam epitaxy. Applied Physics Letters 1987, 50, (14), 903-905.

81. Matthews, J. W.; Crawford, J. L., Accomodation of misfit between singlecrystal films of nickel and copper. Thin Solid Films 1970, 5, (3), 187-198.

82. Prinz, V. Y.; Seleznev, V. A.; Gutakovsky, A. K.; Chehovskiy, A. V.; Preobrazhenskii, V. V.; Putyato, M. A.; Gavrilova, T. A., Free-standing and overgrown InGaAs/GaAs nanotubes, nanohelices and their arrays. Physica E: Low dimensional Systems and Nanostructures 2000, 6, (1-4), 828-831.

83. Ji, H.-X.; Wu, X.-L.; Fan, L.-Z.; Krien, C.; Fiering, I.; Guo, Y.-G.; Mei, Y.; Schmidt, O. G., Self-Wound Composite Nanomembranes as Electrode Materials for Lithium Ion Batteries. Advanced Materials 2010, 22, (41), 4591-4595. 
84. Solovev, A. A.; Xi, W.; Gracias, D. H.; Harazim, S. M.; Deneke, C.; Sanchez, S.; Schmidt, O. G., Self-Propelled Nanotools. ACS Nano 2012, 6, (2), 1751-1756.

85. Xi, W.; Solovev, A. A.; Ananth, A. N.; Gracias, D. H.; Sanchez, S.; Schmidt, O. G., Rolled-up magnetic microdrillers: towards remotely controlled minimally invasive surgery. Nanoscale 2013, 5, (4), 1294-1297.

86. Thurmer, D. J.; Deneke, C.; Mei, Y.; Schmidt, O. G., Process integration of microtubes for fluidic applications. Applied Physics Letters 2006, 89, (22), 223507-3.

87. Songmuang, R.; Rastelli, A.; Mendach, S.; Deneke, C.; Schmidt, O. G., From rolled-up $\mathrm{Si}$ microtubes to $\mathrm{SiOx} / \mathrm{Si}$ optical ring resonators. Microelectronic Engineering 2007, 84, (5-8), 1427-1430.

88. Zander, T.; Deneke, C.; Malachias, A.; Mickel, C.; Metzger, T. H.; Schmidt, O. G., Planar hybrid superlattices by compression of rolled-up nanomembranes. Applied Physics Letters 2009, 94, (5), 053102-3.

89. Qian, M. C.; Fong, C. Y.; Pickett, W. E.; Pask, J. E.; Yang, L. H.; Dag, S., Spin-polarized ballistic transport in a thin superlattice of zinc blende half-metallic compounds. Physical Review B 2005, 71, (1), 012414.

90. Vashaee, D.; Shakouri, A., Improved Thermoelectric Power Factor in MetalBased Superlattices. Physical Review Letters 2004, 92, (10), 106103.

91. Solovev, A. A.; Mei, Y.; Bermúdez Ureña, E.; Huang, G.; Schmidt, O. G., Catalytic Microtubular Jet Engines Self-Propelled by Accumulated Gas Bubbles. Small 2009, 5, (14), 1688-1692.

92. Sanchez, S.; Solovev, A. A.; Schulze, S.; Schmidt, O. G., Controlled manipulation of multiple cells using catalytic microbots. Chemical Communications 2011, 47, (2), 698-700.

93. Cavallo, F.; Songmuang, R.; Schmidt, O. G., Fabrication and electrical characterization of Si-based rolled-up microtubes. Applied Physics Letters 2008, 93, (14), 143113-3. 
94. Smith, E. J.; Schulze, S.; Kiravittaya, S.; Mei, Y.; Sanchez, S.; Schmidt, O. G., Lab-in-a-Tube: Detection of Individual Mouse Cells for Analysis in Flexible SplitWall Microtube Resonator Sensors. Nano Letters 2010, 11, (10), 4037-4042.

95. Esteban Bermúdez, U.; Yongfeng, M.; Emica, C.; Denys, M.; Manfred, A.; Oliver, G. S., Fabrication of ferromagnetic rolled-up microtubes for magnetic sensors on fluids. Journal of Physics D: Applied Physics 2009, 42, (5), 055001.

96. Py, C.; Reverdy, P.; Doppler, L.; Bico, J.; eacute; Roman, B.; icirc; Baroud, C. N., Capillary Origami: Spontaneous Wrapping of a Droplet with an Elastic Sheet. Physical Review Letters 2007, 98, (15), 156103.

97. Maruyama, T.; Minami, H., Light trapping in spherical silicon solar cell module. Solar Energy Materials and Solar Cells 2003, 79, (2), 113-124.

98. Leong, T.; Gu, Z.; Koh, T.; Gracias, D. H., Spatially Controlled Chemistry Using Remotely Guided Nanoliter Scale Containers. Journal of the American Chemical Society 2006, 128, (35), 11336-11337.

99. Azam, A.; Laflin, K.; Jamal, M.; Fernandes, R.; Gracias, D., Self-folding micropatterned polymeric containers. Biomedical Microdevices 2010, 1-8.

100. Randall, C.; Gillespie, A.; Singh, S.; Leong, T.; Gracias, D., Size selective sampling using mobile, 3D nanoporous membranes. Analytical and Bioanalytical Chemistry 2009, 393, (4), 1217-1224.

101. Randall, C. L.; Kalinin, Y. V.; Jamal, M.; Shah, A.; Gracias, D. H., Self-folding immunoprotective cell encapsulation devices. Nanomedicine-Nanotechnology Biology and Medicine 2011, 7, (6), 686-689.

102. Kalinin, Y. V.; Randhawa, J. S.; Gracias, D. H., Three-Dimensional Chemical Patterns for Cellular Self-Organization. Angewandte Chemie International Edition 2011, 50, (11), 2549-2553.

103. Azam, A.; Laflin, K. E.; Jamal, M.; Fernandes, R.; Gracias, D. H., Self-folding micropatterned polymeric containers. Biomedical Microdevices 2011, 13, (1), 51-58. 
104. Cho, J.-H.; Keung, M. D.; Verellen, N.; Lagae, L.; Moshchalkov, V. V.; Van Dorpe, P.; Gracias, D. H., Nanoscale Origami for 3D Optics. Small 2011, 7, (14), 1943-1948.

105. Simpson, B.; Nunnery, G.; Tannenbaum, R.; Kalaitzidou, K., Capture/release ability of thermo-responsive polymer particles. Journal of Materials Chemistry 2010, 20, (17), 3496-3501.

106. Hu, J.; Chen, S., A review of actively moving polymers in textile applications. Journal of Materials Chemistry 2010, 20, (17), 3346-3355.

107. Behl, M.; Lendlein, A., Triple-shape polymers. Journal of Materials Chemistry 2010, 20, (17), 3335-3345.

108. Xie, T., Recent advances in polymer shape memory. Polymer 2011, 52, (22), 4985-5000.

109. Hu, J.; Zhu, Y.; Huang, H.; Lu, J., Recent advances in shape-memory polymers: Structure, mechanism, functionality, modeling and applications. ProgresS in Polymer Science 2012, 37, (12), 1720-1763.

110. Jung, Y.; Cho, J., Application of shape memory polyurethane in orthodontic. Journal of Materials Science: Materials in Medicine 2010, 21, (10), 2881-2886.

111. Ikeda, T.; Ube, T., Photomobile polymer materials: from nano to macro. Materials Today 2011, 14, (10), 480-487.

112. Yamada, M.; Kondo, M.; Miyasato, R.; Naka, Y.; Mamiya, J.-i.; Kinoshita, M.; Shishido, A.; Yu, Y.; Barrett, C. J.; Ikeda, T., Photomobile polymer materials-various three-dimensional movements. Journal of Materials Chemistry 2009, 19, (1), 60-62.

113. Small, W.; Buckley, P. R.; Wilson, T. S.; Benett, W. J.; Hartman, J.; Saloner, D.; Maitland, D. J., Shape Memory Polymer Stent With Expandable Foam: A New Concept for Endovascular Embolization of Fusiform Aneurysms. Biomedical Engineering, IEEE Transactions on 2007, 54, (6), 1157-1160.

114. Xiao, X.; Xie, T.; Cheng, Y.-T., Self-healable graphene polymer composites. Journal of Materials Chemistry 2010, 20, (17), 3508-3514. 
115. Tan, J. L.; Tien, J.; Pirone, D. M.; Gray, D. S.; Bhadriraju, K.; Chen, C. S., Cells lying on a bed of microneedles: An approach to isolate mechanical force. Proceedings of the National Academy of Sciences 2003, 100, (4), 1484-1489.

116. Choquet, D.; Felsenfeld, D. P.; Sheetz, M. P., Extracellular Matrix Rigidity Causes Strengthening of Integrin Cytoskeleton Linkages. Cell 1997, 88, (1), 39-48.

117. Kuribayashi-Shigetomi, K.; Onoe, H.; Takeuchi, S., Cell Origami: Self-Folding of Three-Dimensional Cell-Laden Microstructures Driven by Cell Traction Force. PLoS ONE 2012, 7, (12), e51085.

118. Feinberg, A. W.; Feigel, A.; Shevkoplyas, S. S.; Sheehy, S.; Whitesides, G. M.; Parker, K. K., Muscular thin films for building actuators and powering devices. Science 2007, 317, (5843), 1366-70.

119. SHAYKEWICH, C. F., Proposed Method for Measuring Swelling Pressure of Seeds prior to Germination. Journal of Experimental Botany 1973, 24, (6), 10561061.

120. Harrington, M. J.; Razghandi, K.; Ditsch, F.; Guiducci, L.; Rueggeberg, M.; Dunlop, J. W. C.; Fratzl, P.; Neinhuis, C.; Burgert, I., Origami-like unfolding of hydro-actuated ice plant seed capsules. Nat Commun 2011, 2, 337.

121. Dunlop, J. W. C.; Weinkamer, R.; Fratzl, P., Artful interfaces within biological materials. Materials Today 2011, 14, (3), 70-78.

122. Holmes, D. P.; Crosby, A. J., Snapping surfaces. Advanced Materials 2007, 19, (21), 3589-+.

123. Skotheim, J. M.; Mahadevan, L., Physical Limits and Design Principles for Plant and Fungal Movements. Science 2005, 308, (5726), 1308-1310.

124. Forterre, Y.; Skotheim, J. M.; Dumais, J.; Mahadevan, L., How the Venus flytrap snaps. Nature 2005, 433, (7024), 421-425.

125. Luchnikov, V.; Sydorenko, O.; Stamm, M., Self-rolled polymer and composite polymer/metal micro- and nanotubes with patterned inner walls. Advanced Materials 2005, 17, (9), 1177-1182. 
126. Kumar, K.; Nandan, B.; Luchnikov, V.; Simon, F.; Vyalikh, A.; Scheler, U.; Stamm, M., A Novel Approach for the Fabrication of Silica and Silica/Metal Hybrid Microtubes. Chem Mater 2009, 21, (18), 4282-4287.

127. Kumar, K.; Luchnikov, V.; Nandan, B.; Senkovskyy, V.; Stamm, M., Formation of self-rolled polymer microtubes studied by combinatorial approach. Europ Polym J 2008, 44, (12), 4115-4121.

128. He, H. Y.; Guan, J. J.; Lee, J. L., An oral delivery device based on self-folding hydrogels. Journal of Controlled Release 2006, 110, (2), 339-346.

129. Guan, J. J.; He, H. Y.; Hansford, D. J.; Lee, L. J., Self-folding of threedimensional hydrogel microstructures. Journal of Physical Chemistry B 2005, 109, (49), 23134-23137.

130. Bassik, N.; Abebe, B. T.; Laflin, K. E.; Gracias, D. H., Photolithographically patterned smart hydrogel based bilayer actuators. Polymer 2010, 51, (26), 60936098.

131. Kelby, T. S.; Wang, M.; Huck, W. T. S., Controlled Folding of 2D Au-Polymer Brush Composites into 3D Microstructures. Advanced Functional Materials 2011, $21,(4), 652-657$.

132. Singamaneni, S.; McConney, M. E.; Tsukruk, V. V., Swelling-Induced Folding in Confined Nanoscale Responsive Polymer Gels. ACS Nano 2010, 4, (4), 2327-2337.

133. Kalaitzidou, K.; Crosby, A. J., Adaptive polymer particles. Applied Physics Letters 2008, 93, (4).

134. Tanaka, T.; Okayama, M.; Kitayama, Y.; Kagawa, Y.; Okubo, M., Preparation of "Mushroom-like" Janus Particles by Site-Selective Surface-Initiated Atom Transfer Radical Polymerization in Aqueous Dispersed Systems. Langmuir 2010, 26, (11), 7843-7847.

135. Stoychev, G.; Puretskiy, N.; Ionov, L., Self-folding all-polymer thermoresponsive microcapsules. Soft Matter 2011, 7, 3277-3279

136. Jeong, K.-U.; Jang, J.-H.; Kim, D.-Y.; Nah, C.; Lee, J. H.; Lee, M.-H.; Sun, H.J.; Wang, C.-L.; Cheng, S. Z. D.; Thomas, E. L., Three-dimensional actuators 
transformed from the programmed two-dimensional structures via bending, twisting and folding mechanisms. Journal of Materials Chemistry 2011, 21, 6824-6830.

137. Zhang, X.; Pint, C. L.; Lee, M. H.; Schubert, B. E.; Jamshidi, A.; Takei, K.; Ko, H.; Gillies, A.; Bardhan, R.; Urban, J. J.; Wu, M.; Fearing, R.; Javey, A., Opticallyand Thermally-Responsive Programmable Materials Based on Carbon NanotubeHydrogel Polymer Composites. Nano Letters 2011, 11, (8), 3239-3244.

138. Kim, J.; Hanna, J. A.; Byun, M.; Santangelo, C. D.; Hayward, R. C., Designing Responsive Buckled Surfaces by Halftone Gel Lithography. Science 2012, 335, (6073), 1201-1205.

139. Jamal, M.; Zarafshar, A. M.; Gracias, D. H., Differentially photo-crosslinked polymers enable self-assembling microfluidics. Nature Communications 2011, 2.

140. Sameoto, D.; Tsang, S. H.; Foulds, I. G.; Lee, S. W.; Parameswaran, M., Control of the out-of-plane curvature in SU-8 compliant microstructures by exposure dose and baking times. Journal of Micromechanics and Microengineering 2007, 17, (5), 1093.

141. Ru, F.; Richard, J. F., Influence of processing conditions on the thermal and mechanical properties of SU8 negative photoresist coatings. Journal of Micromechanics and Microengineering 2003, 13, (1), 80.

142. Stephan, K.; Gabriela, B.; Michael, L.; Daniel, H.; Anja, B., Processing of thin SU-8 films. Journal of Micromechanics and Microengineering 2008, 18, (12), 125020.

143. Jeong, K.-U.; Jang, J.-H.; Kim, D.-Y.; Nah, C.; Lee, J. H.; Lee, M.-H.; Sun, H.J.; Wang, C.-L.; Cheng, S. Z. D.; Thomas, E. L., Three-dimensional actuators transformed from the programmed two-dimensional structures via bending, twisting and folding mechanisms. Journal of Materials Chemistry 2011.

144. Holmes, D. P.; Roche, M.; Sinha, T.; Stone, H. A., Bending and twisting of soft materials by non-homogenous swelling. Soft Matter 2011, 7, (11), 5188-5193.

145. Luchnikov, V. A.; Saito, Y.; Tzanis, L., A Novel Fibrous Material Created by Self-Rolling of a Patterned Polymer Thin Film. Macromolecular Rapid Communications 2012, 33, (16), 1404-1408. 
146. Betancourt, T.; Brannon-Peppas, L., Micro- and nanofabrication methods in nanotechnological medical and pharmaceutical devices. International journal of nanomedicine 2006, 1, (4), 483-495.

147. Voldman, J.; Gray, M. L.; Schmidt, M. A., Microfabrication in biology and medicine. Annual Review of Biomedical Engineering 1999, 1, 401-425.

148. Jackman, R. J.; Duffy, D. C.; Ostuni, E.; Willmore, N. D.; Whitesides, G. M., Fabricating Large Arrays of Microwells with Arbitrary Dimensions and Filling Them Using Discontinuous Dewetting. Analytical Chemistry 1998, 70, (11), 2280-2287.

149. Xia, Y.; Whitesides, G. M., SOFT LITHOGRAPHY. Annual Review of Materials Science 1998, 28, (1), 153-184.

150. Zhao, B.; Brittain, W. J., Polymer brushes: surface-immobilized macromolecules. Progress in Polymer Science 2000, 25, (5), 677-710.

151. Zdyrko, B.; Luzinov, I., Polymer Brushes by the "Grafting to" Method. Macromolecular Rapid Communications 2011, 32, (12), 859-869.

152. Wang, M.; Comrie, J. E.; Bai, Y.; He, X.; Guo, S.; Huck, W. T. S., Formation of Hierarchically Structured Thin Films. Advanced Functional Materials 2009, 19, (14), 2236-2243.

153. Kumar, K.; Nandan, B.; Luchnikov, V.; Simon, F.; Vyalikh, A.; Scheler, U.; Stamm, M., A Novel Approach for the Fabrication of Silica and Silica/Metal Hybrid Microtubes. Chemistry of Materials 2009, 21, (18), 4282-4287.

154. Kumar, K.; Luchnikov, V.; Nandan, B.; Senkovskyy, V.; Stamm, M., Formation of self-rolled polymer microtubes studied by combinatorial approach. European Polymer Journal 2008, 44, (12), 4115-4121.

155. Laflin, K. E.; Morris, C. J.; Muqeem, T.; Gracias, D. H., Laser triggered sequential folding of microstructures. Applied Physics Letters 2012, 101, (13), 131901

156. Liang, H. Y.; Mahadevan, L., Growth, geometry, and mechanics of a blooming lily. Proceedings of the National Academy of Sciences of the United States of America 2011, 108, (14), 5516-5521. 
157. Liang, H.; Mahadevan, L., The shape of a long leaf. Proceedings of the National Academy of Sciences of the United States of America 2009, 106, (52), $22049-22054$.

158. Ionov, L.; Zdyrko, B.; Sidorenko, A.; Minko, S.; Klep, V.; Luzinov, I.; Stamm, M., Gradient Polymer Layers by "Grafting To" Approach. Macromolecular Rapid Communications 2004, 25, (1), 360-365.

159. Hawkes, P. W.; Spence, J. C. H., Science of Microscopy. In 2008; Vol. 1.

160. Stuart, M. A. C.; Huck, W. T. S.; Genzer, J.; Muller, M.; Ober, C.; Stamm, M.; Sukhorukov, G. B.; Szleifer, I.; Tsukruk, V. V.; Urban, M.; Winnik, F.; Zauscher, S.; Luzinov, I.; Minko, S., Emerging applications of stimuli-responsive polymer materials. Nat Mater 2010, 9, (2), 101-113.

161. Stoychev, G.; Zakharchenko, S.; Turcaud, S.; Dunlop, J. W. C.; Ionov, L., Shape-Programmed Folding of Stimuli-Responsive Polymer Bilayers. ACS Nano 2012, 6, (5), 3925-3934.

162. Stoychev, G.; Puretskiy, N.; Ionov, L., Self-folding all-polymer thermoresponsive microcapsules. Soft Matter 2011, 7, (7), 3277-3279.

163. Marder, M.; Sharon, E.; Smith, S.; Roman, B., Theory of edges of leaves. EPL (Europhysics Letters) 2003, 62, (4), 498.

164. Audoly, B.; Boudaoud, A., 'Ruban à godets': an elastic model for ripples in plant leaves. Comptes Rendus Mécanique 2002, 330, (12), 831-836.

165. Stoychev, G.; Turcaud, S.; Dunlop, J. W. C.; Ionov, L., Hierarchical Multi-Step Folding of Polymer Bilayers. Advanced Functional Materials 2013, 23, (18), 22952300 . 


\section{Curriculum Vitae}

Personal infromation

Forename

Georgi

Surname

Stoychev

Date of birth

22.10 .1985

Place of birth

Veliko Turnovo, Bulgarien

Education

09.2009-08.2013

$\mathrm{PhD}$ Thesis at TU Dresden/Leibniz Institute of Polymer Research Dresden: Shape-programmed Folding of Stimuli-Responsive Polymer Bilayers

Sep 2004 - Jun 2009

Lomonosov Moscow State University

M.Sci in Chemistry, GPA 4.85 of 5.00

1999-2003

High School of Mathematics and Natural Sciences "Vasil Drumev"

Research projects and empolyments

Feb 2005-May 2005

Research fellow at the Lomonosov Moscow State University, Department of Chemistry (Dr. Nadezhda D. Mitrofanova) ; "Synthesis of $\mathrm{SO}_{2} \mathrm{Cl}_{2}$ "

Research fellow at the Lomonosov Moscow State

Feb 2007-May 2007 University, Department of Chemistry (Dr. Elvira V. Uglova); "Synthesis of 5,7-dinitro-8-(8quinolinethio)-quinoline"

Internship at the Newcastle Technology Centre,

Jun 2008- Aug 2008 BHP Billiton, Australia (Prof. Robert Sumner and Dr. Michael Wheatley); "Goethite Settling"

Research fellow at the Lomonosov Moscow State University, Department of Chemistry (Dr.

Oct 2004 - May $2009 \quad$ Ekaterina A. Litmanovich); "Phase behaviour of polymer-polymer and polymer-surfactant aqueous solutions"

Sep 2009 - present

Guest researcher at the Leibniz Institute of Polymer Research Dresden 


\section{Advanced training}

2010

$6^{\text {th }}$ Cycle of the PhD School in Focused Ion and Electron Beams, Teaching Week I, Belvaux, Luxembourg, November 14-19, 2010.

EMAT - ESTEEM Winter Workshop on

2011

Transmission Electron Microscopy 2011, University of Antwerp, Antwerp, Belgium, 17 - 28 January, 2011.

$6^{\text {th }}$ Cycle of the PhD School in Focused Ion and

2011 Electron Beams, Teaching Week II, Belvaux, Luxembourg, 28 $8^{\text {th }}$ of February $-4^{\text {th }}$ of March, 2011.

Lab Course on High-Resolution Transmission

2012 Electron Microscopy and Hologrphy, Triebenberg Laboratory, Germany, 12 ${ }^{\text {th- }} 23^{\text {rd }}$ March, 2012

2013

$1^{\text {st }}$ International CryoEM Workshop, Eindhoven, The Netherlands, 28th of January 2013-1st of February 2013

\section{Personal skills and Competences}

Languages:

German (very good)

English (fluent)

Russian (fluent)

Bulgarian (native)

Software:

Safe handling of MS-Office software, ChemOffice, Origin 8.x, CorelDRAW 6

Methods:

Dynamic and static light scattering

Capillary and rotational viscometry

Electroacoustosizing

Null-ellipsometry

Scanning electron microscoy (SEM) and cryoSEM

Transmission electron microscopy (TEM) and cryoTEM 
Optical/fluorescence microscopy

Dip-coating and spin-coating

Polymer synthesis via radical polymerization, ATRP, AGET-ATRP

Surface modification via "grafting-from" approach 


\section{List of Publications}

\section{Journal articles:}

Zakharchenko, S.; Puretskiy, N.; Stoychev, G.; Stamm, M.; Ionov, L.: Temperature controlled encapsulation and release using partially biodegradable thermo-magneto-sensitive self-rolling tubes. Soft Matter 2010, 6, (12), 2633-2636

Puretskiy, N.; Stoychev, G.; Stamm, M.; Ionov, L.: Switchable Surfaces Based on Freely Floating Colloidal Particles. Acs Applied Materials \& Interfaces 2010, 2, (10), 2944-2948

Synytska, A.; Svetushkina, E.; Puretskiy, N.; Stoychev, G.; Berger, S.; Ionov, L.; Bellmann, C.; Eichhorn, K. J.; Stamm, M: Biocompatible polymeric materials with switchable adhesion properties. Soft Matter 2010, 6, (23), 5907-5914

Stoychev, G.; Puretskiy, N.; Ionov, L.: Self-folding all-polymer thermoresponsive microcapsules. Soft Matter, 2011, 7, (7), 3277-3279

Stoychev, G.; Zakharchenko, S.; Turcaud, S.; Dunlop, J. W. C.; Ionov, L.: Shape-Programmed Folding of Stimuli-Responsive Polymer Bilayers. ACS Nano, 2012, 6, (5), 3925-3934

Puretskiy, N.; Stoychev, G.; Synytska, A.; Ionov, L.: Surfaces with Selfrepairable Ultrahydrophobicity Based on Self-organizing Freely Floating Colloidal Particles. Langmuir, 2012, 28, (8), 3679-3682

Raguzin, I.; Stoychev, G.; Stamm, M.; Ionov, L.: Single molecule investigation of complexes of oppositely charged bottle brushes. Soft Matter, 2013, 9, (2), 359-364 
Stoychev, G.; Turcaud, S.; Dunlop, J. W. C.; Ionov, L.: Hierarchical multi-step folding of polymer bilayers. Advanced Functional Materials, 2013, 23, (18), $2295-2300$

Zakharchenko S.; Puretskiy, N.; Stoychev, G.; Waurisch C.; Hickey S.; Eychmüller A.; Sommer J.-U.; Ionov L.: Stimuli-responsive hierarchically self-assembled 3D porous polymer-based structures with aligned pores. Journal of Material Chemistry B, 2013, 1, 1786-1793

\section{Contribution to conferences:}

$13^{\text {th }}$ Dresden Polymer Discussion and $8^{\text {th }}$ Max Bergmann Symposium "Molecular Bioengineering Meets Polymer Science", April 1-4, 2012, Meissen (Poster)

MSE Congress - "Shape-programmed folding of stimuli-responsive polymer bilayers", 25-27 September 2012, Darmstadt, Germany (Poster)

DPG-Frühjahrstagung - "Hierarchical folding of polymer bilayers", 10-15 March 2013, Regensburg, Germany (Poster) 


\section{Acknowledgements}

First of all, I wish to express my gratitude to my supervisor Professor Manfred Stamm for his guidance and support throughout this research. I am deeply thankful for the guidance, both scientific and moral, for the encouragement, fruitful discussions and friendly support by Dr. Leonid Ionov who co-supervised my work. I'm especially grateful to him for not giving up on me in times of doubt and trouble when his reassuring advice helped me stay strong and continue my PhD. I owe also special gratitude to Dr. Petr Formanek who unraveled the fascinating world of electron microscopy to me. He was always ready to help with and explain my errors and never lost patience teaching me.

I am very thankful to my colleagues Nikolay Puretskiy, Ivan Raguzin and Svetlana Zakharchenko for their help with my work and for being friends.

I would like to thank Dr. Alla Synytska for fruitful and positive discussions and for letting me work in her laboratory from time to time. I'm thankful to Michael Göbel for his help with cryoSEM and for the interesting discussions about electron microscopy. In the course of this, I'd like to say thank you to Leonard Schellkopf for our highly beneficial conversations on TEM and cryoTEM, which improved my skills in these complicated techniques.

I also would like to thank Dr. Mark Leaver for being a trustworthy friend and climbing partner and for teaching me English. His precious recommendations in improving the text quality of this work are mostly appreciated.

I would like to deeply thank my girlfriend Alina Kirillova for her inexhaustible trust in me, for her support, for her help in improving my 
language skills and for keeping my love for science alive. Her invaluable aid in correcting the grammar, spelling and design of my thesis is gratefully acknowledged.

Finally I'd like to thank my beloved parents Zemfira and Valeri who always believed in me and were supportive both in good and hard times and my brother Stoyan whose positive attitude toward life in general charged me with brisk optimism. 